\title{
Inverse problems: seeing the unseen
}

\author{
Gunther Uhlmann
}

Received: 1 March 2014 / Accepted: 5 May 2014 / Published online: 5 June 2014

(C) The Author(s) 2014. This article is published with open access at SpringerLink.com

\begin{abstract}
This survey article deals mainly with two inverse problems and the relation between them. The first inverse problem we consider is whether one can determine the electrical conductivity of a medium by making voltage and current measurements at the boundary. This is called electrical impedance tomography and also Calderón's problem since the famous analyst proposed it in the mathematical literature (Calderón in On an inverse boundary value problem. Seminar on numerical analysis and its applications to continuum physics (Rio de Janeiro, 1980), Soc Brasil Mat. Rio de Janeiro, pp. 65-73, 1980). The second is on travel time tomography. The question is whether one can determine the anisotropic index of refraction of a medium by measuring the travel times of waves going through the medium. This can be recast as a geometry problem, the boundary rigidity problem. Can we determine a Riemannian metric of a compact Riemannian manifold with boundary by measuring the distance function between boundary points? These two inverse problems concern visibility, that is whether we can determine the internal properties of a medium by making measurements at the boundary. The last topic of this paper considers the opposite issue: invisibility: Can one make objects invisible to different types of waves, including light?
\end{abstract}

Communicated by Ari Laptev.

Gunther Uhlmann: Simons Fellowship.

G. Uhlmann $(\varangle)$

Department of Mathematics, University of Washington,

Seattle, WA 98195, USA

e-mail: gunther@math.washington.edu 


\section{Calderón's problem}

\subsection{Introduction}

In 1980 Calderón published a short paper entitled "On an inverse boundary value problem" [37]. This pioneer contribution motivated many developments in inverse problems, in particular in the construction of "complex geometrical optics" solutions of partial differential equations to solve several inverse problems. We survey some these developments in this paper. In his talk at the ICM in Berlin in 1998 the author proposed 7 open problems [199] on this subject. This section is to a large extent a report on the progress made in solving these problems.

The problem that Calderón considered was whether one can determine the electrical conductivity of a medium by making voltage and current measurements at the boundary of the medium. This inverse method is known as Electrical Impedance Tomography (EIT). Calderón was motivated by oil prospection. In the 40's he worked as an engineer for Yacimientos Petroliferos Fiscales (YPF), the state oil company of Argentina, and he thought about this problem then although he did not publish his results until many years later. For use of electrical methods in geophysical prospection see [208]. Parenthetically Calderón said in his speech accepting the "Doctor Honoris Causa" of the Universidad Autónoma de Madrid that his work at YPF had been very interesting but he was not well treated there; he would have stayed at YPF otherwise [38]. It goes without saying that the bad treatment of Calderón by YPF was very fortunate for Mathematics! EIT also arises in medical imaging given that human organs and tissues have quite different conductivities [103]. One exciting potential application is the early diagnosis of breast cancer [210]. The conductivity of a malignant breast tumor is typically 0.2 mho which is significantly higher than normal tissue which has been typically measured at 0.03 mho. Another application is to monitor pulmonary functions [94]. See the book [79] and the issue of Physiological Measurement [80] for other medical imaging applications of EIT. This inverse method has also been used to detect leaks from buried pipes [102]. For other reviews see [29,43] and [74]. We now describe more precisely the mathematical problem. Let $\Omega \subseteq \mathbb{R}^{n}$ be a bounded domain with smooth boundary (many of the results we will describe are valid for domains with Lipschitz boundaries). The electrical conductivity of $\Omega$ is represented by a bounded and positive function $\gamma(x)$. In the absence of sinks or sources of current the equation for the potential is given by

$$
\nabla \cdot(\gamma \nabla u)=0 \text { in } \Omega
$$

since, by Ohm's law, $\gamma \nabla u$ represents the current flux. Given a potential $f \in H^{\frac{1}{2}}(\partial \Omega)$ on the boundary the induced potential $u \in H^{1}(\Omega)$ solves the Dirichlet problem

$$
\begin{aligned}
\nabla \cdot(\gamma \nabla u) & =0 \text { in } \Omega, \\
\left.u\right|_{\partial \Omega} & =f .
\end{aligned}
$$


The Dirichlet to Neumann map, or voltage to current map, is given by

$$
\Lambda_{\gamma}(f)=\left.\left(\gamma \frac{\partial u}{\partial v}\right)\right|_{\partial \Omega}
$$

where $v$ denotes the unit outer normal to $\partial \Omega$. The inverse problem is to determine $\gamma$ knowing $\Lambda_{\gamma}$. It is difficult to find a systematic way of prescribing voltage measurements at the boundary to be able to find the conductivity. Calderón took instead a different route. Using the divergence theorem we have

$$
Q_{\gamma}(f):=\int_{\Omega} \gamma|\nabla u|^{2} d x=\int_{\partial \Omega} \Lambda_{\gamma}(f) f d S
$$

where $d S$ denotes surface measure and $u$ is the solution of (2). In other words $Q_{\gamma}(f)$ is the quadratic form associated to the linear map $\Lambda_{\gamma}(f)$, and to know $\Lambda_{\gamma}(f)$ or $Q_{\gamma}(f)$ for all $f \in H^{\frac{1}{2}}(\partial \Omega)$ is equivalent. $Q_{\gamma}(f)$ measures the energy needed to maintain the potential $f$ at the boundary. Calderón's point of view is that if one looks at $Q_{\gamma}(f)$ the problem is changed to finding enough solutions $u \in H^{1}(\Omega)$ of the Eq. (1) in order to find $\gamma$ in the interior. These are the complex geometrical optics (CGO) solutions considered in Sect. 1.3. A short summary of the contents of this section is as follows. In Sect. 1.2 we describe results about uniqueness, stability and reconstruction, for the boundary values of a conductivity and its normal derivative. In Sect. 1.3 we describe the construction by Sylvester and Uhlmann [190,191] of CGO solutions for the Schrödinger equation associated to a bounded potential. These solutions behave like Calderón's complex exponential solutions for large complex frequencies. In Sect. 1.4 we use these solutions to prove, in dimension $n \geq 3$, a global identifiability result, stability estimates and a reconstruction method for the inverse problem. We also describe an extension of the identifiability result to nonlinear conductivities [184] and give other applications of CGO solutions. In Sect. 1.5 we consider the partial data problem, that is the case when the DN map is measured on a part of the boundary. We describe the results of [107] for the non-linear problem in dimension three or larger. This uses a larger class of CGO solutions, having a non-linear phase function that are constructed using Carleman estimates. We also review the article [49] for the linearized problem with partial data. In Sect. 1.6 we consider the two dimensional case. In particular we describe briefly the recent work of Astala and Päivärinta proving uniqueness for bounded measurable coefficients, and the work of Bukhgeim proving uniqueness for a potential from Cauchy data associated to the Schrödinger equation. Finally we consider the work of Imanuvilov, Uhlmann and Yamamoto on the partial data problem in two dimensions [87,88]. These sections deal with the case of isotropic conductivities. In Sect. 1.7 we consider the case of anisotropic conductivities, i.e. the conductivity depends also on direction. In two dimensions that there has been substantial progress in the understanding of anisotropic problems since one can usually reduce the problem to the isotropic case by using isothermal coordinates. In dimension three the problem as pointed out in [121] is of geometric nature. We review the results of $[52,124]$. 


\subsection{Boundary determination}

Kohn and Vogelius proved the following identifiability result at the boundary [114].

Theorem 1.1 Let $\gamma_{i} \in C^{\infty}(\bar{\Omega})$ be strictly positive. Assume $\Lambda_{\gamma_{1}}=\Lambda_{\gamma_{2}}$. Then

$$
\left.\partial^{\alpha} \gamma_{1}\right|_{\partial \Omega}=\left.\partial^{\alpha} \gamma_{2}\right|_{\partial \Omega}, \quad \forall|\alpha| \text {. }
$$

This settled the identifiability question for the non-linear problem in the realanalytic category. They extended the identifiability result to piecewise real-analytic conductivities in [115].

Sketch of proof of Theorem 1.1. We outline an alternative proof to the one given by Kohn and Vogelius of 1.1. In the case $\gamma \in C^{\infty}(\bar{\Omega})$ we know, by another result of Calderón [39], that $\Lambda_{\gamma}$ is a classical pseudodifferential operator of order 1. Let $\left(x^{\prime}, x^{n}\right)$ be coordinates near a point $x_{0} \in \partial \Omega$ so that the boundary is given by $x^{n}=0$. The function $\lambda_{\gamma}\left(x^{\prime}, \xi^{\prime}\right)$ denotes the full symbol of $\Lambda_{\gamma}$ in these coordinates. It was proved in [192] that

$$
\lambda_{\gamma}\left(x^{\prime}, \xi^{\prime}\right)=\gamma\left(x^{\prime}, 0\right)\left|\xi^{\prime}\right|+a_{0}\left(x^{\prime}, \xi^{\prime}\right)+r\left(x^{\prime}, \xi^{\prime}\right)
$$

where $a_{0}\left(x^{\prime}, \xi^{\prime}\right)$ is homogeneous of degree 0 in $\xi^{\prime}$ and is determined by the normal derivative of $\gamma$ at the boundary and tangential derivatives of $\gamma$ at the boundary. The term $r\left(x^{\prime}, \xi^{\prime}\right)$ is a classical symbol of order -1 . Then $\left.\gamma\right|_{\partial \Omega}$ is determined by the principal symbol of $\Lambda_{\gamma}$ and $\left.\frac{\partial \gamma}{\partial x^{n}}\right|_{\partial \Omega}$ by the principal symbol and the term homogeneous of degree 0 in the expansion of the full symbol of $\Lambda_{\gamma}$. More generally the higher order normal derivatives of the conductivity at the boundary can be determined recursively. In Lee and Uhlmann [121] one can find a general approach to the calculation of the full symbol of the Dirichlet to Neumann map that applies to more general situations. We note that this gives also a reconstruction procedure. We first can reconstruct $\gamma$ at the boundary since $\left.\gamma\right|_{\partial \Omega}\left|\xi^{\prime}\right|$ is the principal symbol of $\Lambda_{\gamma}$ [see (5)]. In other words in coordinates $\left(x^{\prime}, x^{n}\right)$ so that $\partial \Omega$ is locally given by $x^{n}=0$ we have

$$
\gamma\left(x^{\prime}, 0\right) a\left(x^{\prime}\right)=\lim _{s \rightarrow \infty} e^{-i s<x^{\prime}, \omega^{\prime}>} \frac{1}{s} \Lambda_{\gamma}\left(e^{i s<x^{\prime}, \omega^{\prime}>} a\left(x^{\prime}\right)\right)
$$

with $\omega^{\prime} \in \mathbb{R}^{n-1}$ and $\left|\omega^{\prime}\right|=1$ and $a$ a smooth and compactly supported function. In a similar fashion, using (5), one can find $\left.\frac{\partial \gamma}{\partial v}\right|_{\partial \Omega}$ by computing the principal symbol of $\left(\Lambda_{\gamma}-\left.\gamma\right|_{\partial \Omega} \Lambda_{1}\right)$ where $\Lambda_{1}$ denotes the Dirichlet to Neumann map associated to the conductivity 1 . The other terms can be reconstructed recursively in a similar fashion. We also observe, by taking an appropriate cut-off function $a$ above, that this procedure is local, that is we only need to know the DN map in an open set of the boundary to determine the Taylor series of the conductivity in that open set. This method also leads to stability estimates at the boundary [192]. 
Theorem 1.2 Suppose that $\gamma_{1}$ and $\gamma_{2}$ are $C^{\infty}$ functions on $\bar{\Omega} \subseteq \mathbb{R}^{n}$ satisfying
i) $0<\frac{1}{E} \leq \gamma_{i} \leq E$
ii) $\left\|\gamma_{i}\right\|_{C^{2}(\bar{\Omega})} \leq E$

Given any $0<\sigma<\frac{1}{n+1}$, there exists $C=C(\Omega, E, n, \sigma)$ such that

$$
\left\|\gamma_{1}-\gamma_{2}\right\|_{L^{\infty}(\partial \Omega)} \leq C\left\|\Lambda_{\gamma_{1}}-\Lambda_{\gamma_{2}}\right\|_{\frac{1}{2}, \frac{-1}{2}}
$$

and

$$
\left\|\frac{\partial \gamma_{1}}{\partial v}-\frac{\partial \gamma_{2}}{\partial v}\right\|_{L^{\infty}(\partial \Omega)} \leq C\left\|\Lambda_{\gamma_{1}}-\Lambda_{\gamma_{2}}\right\|_{\frac{1}{2}, \frac{-1}{2}}^{\sigma} .
$$

This result implies that we don't need the conductivity to be smooth to determine the conductivity and its normal derivative at the boundary. In the case $\gamma$ is continuous on $\bar{\Omega}$ we can determine $\gamma$ at the boundary by using the stability estimate (6) and an approximation argument. In the case that $\gamma \in C^{1}(\bar{\Omega})$ we can determine, knowing the DN map, $\gamma$ and its normal derivative at the boundary using the estimate (7) above and an approximation argument. For other results and approaches to boundary determination of the conductivity see $[5,31,135,141]$. In one way or another the boundary determination involves testing the DN map against highly oscillatory functions at the boundary.

\subsection{Complex geometrical optics solutions with a linear phase}

Motivated by Calderón exponential solutions used in [37] (see [92] for a numerical study) in the study of the linearized problem at a constant conductivity, Sylvester and Uhlmann [190,191] constructed in dimension $n \geq 2$ complex geometrical optics (CGO) solutions of the conductivity equation for $C^{2}$ conductivities that behave like Calderón exponential solutions for large frequencies. This can be reduced to constructing solutions in the whole space (by extending $\gamma=1$ outside a large ball containing $\Omega$ ) for the Schrödinger equation with potential. We describe this more precisely below. Let $\gamma \in C^{2}\left(\mathbb{R}^{n}\right), \gamma$ strictly positive in $\mathbb{R}^{n}$ and $\gamma=1$ for $|x| \geq R, R>0$. Let $L_{\gamma} u=\nabla \cdot \gamma \nabla u$. Then we have

$$
\gamma^{-\frac{1}{2}} L_{\gamma}\left(\gamma^{-\frac{1}{2}}\right)=\Delta-q
$$

where

$$
q=\frac{\Delta \sqrt{\gamma}}{\sqrt{\gamma}}
$$

Therefore, to construct solutions of $L_{\gamma} u=0$ in $\mathbb{R}^{n}$ it is enough to construct solutions of the Schrödinger equation $(\Delta-q) u=0$ with $q$ of the form (9). The next result proven in $[190,191]$ states the existence of complex geometrical optics solutions for the Schrödinger equation associated to any bounded and compactly supported potential. 
Theorem 1.3 Let $q \in L^{\infty}\left(\mathbb{R}^{n}\right), n \geq 2$, with $q(x)=0$ for $|x| \geq R>0$. Let $-1<\delta<0$. There exists $\epsilon(\delta)$ and such that for every $\rho \in \mathbb{C}^{n}$ satisfying

$$
\rho \cdot \rho=0
$$

and

$$
\frac{\left\|\left(1+|x|^{2}\right)^{1 / 2} q\right\|_{L^{\infty}\left(\mathbb{R}^{n}\right)}+1}{|\rho|} \leq \epsilon
$$

there exists a unique solution to

$$
(\Delta-q) u=0
$$

of the form

$$
u=e^{x \cdot \rho}\left(1+\psi_{q}(x, \rho)\right)
$$

with $\psi_{q}(\cdot, \rho) \in L_{\delta}^{2}\left(\mathbb{R}^{n}\right)$. Moreover $\psi_{q}(\cdot, \rho) \in H_{\delta}^{2}\left(\mathbb{R}^{n}\right)$ and for $0 \leq s \leq 2$ there exists $C=C(n, s, \delta)>0$ such that

$$
\left\|\psi_{q}(\cdot, \rho)\right\|_{H_{\delta}^{s}} \leq \frac{C}{|\rho|^{1-s}} .
$$

Here

$$
L_{\delta}^{2}\left(\mathbb{R}^{n}\right)=\left\{f ; \int\left(1+|x|^{2}\right)^{\delta}|f(x)|^{2} d x<\infty\right\}
$$

with the norm given by $\|f\|_{L_{\delta}^{2}}^{2}=\int\left(1+|x|^{2}\right)^{\delta}|f(x)|^{2} d x$ and $H_{\delta}^{m}\left(\mathbb{R}^{n}\right)$ denotes the corresponding Sobolev space. Note that for large $|\rho|$ these solutions behave like Calderón's exponential solutions $e^{x \cdot \rho}$. The equation for $\psi_{q}$ is given by

$$
(\Delta+2 \rho \cdot \nabla) \psi_{q}=q\left(1+\psi_{q}\right)
$$

The Eq. (12) is solved by constructing an inverse for $(\Delta+2 \rho \cdot \nabla)$ and solving the integral equation

$$
\psi_{q}=(\Delta+2 \rho \cdot \nabla)^{-1}\left(q\left(1+\psi_{q}\right)\right)
$$

Lemma 1.4 Let $-1<\delta<0, \quad 0 \leq s \leq 1$. Let $\rho \in \mathbb{C}^{n}-0, \rho \cdot \rho=0$. Let $f \in L_{\delta+1}^{2}\left(\mathbb{R}^{n}\right)$. Then there exists a unique solution $u_{\rho} \in L_{\delta}^{2}\left(\mathbb{R}^{n}\right)$ of the equation

$$
\Delta_{\rho} u_{\rho}:=(\Delta+2 \rho \cdot \nabla) u_{\rho}=f
$$


Moreover $u_{\rho} \in H_{\delta}^{2}\left(\mathbb{R}^{n}\right)$ and

$$
\left\|u_{\rho}\right\|_{H_{\delta}^{s}\left(\mathbb{R}^{n}\right)} \leq \frac{C_{s, \delta}\|f\|_{L_{\delta+1}^{2}}}{|\rho|^{1-s}}
$$

for $0 \leq s \leq 2$ and for some constant $C_{s, \delta}>0$.

The integral equation (12) can then be solved in $L_{\delta}^{2}\left(\mathbb{R}^{n}\right)$ for large $|\rho|$ since

$$
\left(I-(\Delta+2 \rho \cdot \nabla)^{-1} q\right) \psi_{q}=(\Delta+2 \rho \cdot \nabla)^{-1} q
$$

and $\left\|(\Delta+2 \rho \cdot \nabla)^{-1} q\right\|_{L_{\delta}^{2} \rightarrow L_{\delta}^{2}} \leq \frac{C}{|\rho|}$ for some $C>0$ where $\|\cdot\|_{L_{\delta}^{2} \rightarrow L_{\delta}^{2}}$ denotes the operator norm between $L_{\delta}^{2}\left(\mathbb{R}^{n}\right)$ and $L_{\delta}^{2}\left(\mathbb{R}^{n}\right)$. We will not give details of the proof of Lemma 1.4 here. We refer to the papers $[190,191]$. We note that there has been other approaches to construct CGO solutions for the Schrödinger equation [73,97]. These constructions don't give uniqueness of the CGO solutions that are used in the reconstruction method of the conductivity from the DN map (see Sect. 1.4.5). If 0 is not a Dirichlet eigenvalue for the Schrödinger equation we can also define the DN map

$$
\Lambda_{q}(f)=\left.\frac{\partial u}{\partial v}\right|_{\partial \Omega}
$$

where $u$ solves

$$
(\Delta-q) u=0 ;\left.\quad u\right|_{\partial \Omega}=f .
$$

More generally we can define the set of Cauchy data for the Schrödinger equation. Let $q \in L^{\infty}(\Omega)$. We define the Cauchy data as the set

$$
\mathcal{C}_{q}=\left\{\left(\left.u\right|_{\partial \Omega},\left.\frac{\partial u}{\partial v}\right|_{\partial \Omega}\right)\right\}
$$

where $u \in H^{1}(\Omega)$ is a solution of

$$
(\Delta-q) u=0 \text { in } \Omega .
$$

We have $\mathcal{C}_{q} \subseteq H^{\frac{1}{2}}(\partial \Omega) \times H^{-\frac{1}{2}}(\partial \Omega)$. If 0 is not a Dirichlet eigenvalue of $\Delta-q$, then in fact $\mathcal{C}_{q}$ is a graph, namely

$$
\mathcal{C}_{q}=\left\{\left(f, \Lambda_{q}(f)\right) \in H^{\frac{1}{2}}(\partial \Omega) \times H^{-\frac{1}{2}}(\partial \Omega)\right\} .
$$

Complex geometrical optics for first order equations and systems under different regularity assumptions of the coefficients have been constructed in [142,144,162,163,197]. For the case of the magnetic Schrödinger operator unique identifiability of the magnetic 
field and the electrical potential was shown in [120] assuming that both the electrical potential and magnetic potential are both just bounded. We refer to the article a more up to date developments on this topic and the references given there.

\subsection{The Calderón problem in dimension $n \geq 3$}

In this section we summarize some of the basic theoretical results for Calderón's problem in dimension three or higher.

\subsubsection{Uniqueness}

The identifiability question was resolved in [190] for smooth enough conductivities. The result is

Theorem 1.5 Let $\gamma_{i} \in C^{2}(\bar{\Omega}), \gamma_{i}$ strictly positive, $i=1$, 2. If $\Lambda_{\gamma_{1}}=\Lambda_{\gamma_{2}}$ then $\gamma_{1}=\gamma_{2}$ in $\bar{\Omega}$.

In dimension $n \geq 3$ this result is a consequence of a more general result. Let $q \in L^{\infty}(\Omega)$.

Theorem 1.6 Let $q_{i} \in L^{\infty}(\Omega), i=1,2$. Assume $\mathcal{C}_{q_{1}}=\mathcal{C}_{q_{2}}$, then $q_{1}=q_{2}$.

We now show that Theorem 1.6 implies Theorem 1.5. Using (8) we have

$$
\mathcal{C}_{q_{i}}=\left\{\left(f,\left(\left.\left.\frac{1}{2} \gamma_{i}^{-1}\right|_{\partial \Omega} \frac{\partial \gamma_{i}}{\partial \nu}\right|_{\partial \Omega}\right) f+\left.\gamma_{i}^{-\frac{1}{2}}\right|_{\partial \Omega} \Lambda_{\gamma_{i}}\left(\left.\gamma^{-\frac{1}{2}}\right|_{\partial \Omega} f\right)\right), \quad f \in H^{\frac{1}{2}}(\partial \Omega)\right\} .
$$

Then we conclude $\mathcal{C}_{q_{1}}=\mathcal{C}_{q_{2}}$ using the boundary identifiability result of Kohn and Vogelius [114] and its extension [192].

Proof of Theorem 1.6 Let $u_{i} \in H^{1}(\Omega)$ be a solution of

$$
\left(\Delta-q_{i}\right) u_{i}=0 \text { in } \Omega, \quad i=1,2 .
$$

Then using the divergence theorem we have

$$
\int_{\Omega}\left(q_{1}-q_{2}\right) u_{1} u_{2} d x=\int_{\partial \Omega}\left(\frac{\partial u_{1}}{\partial v} u_{2}-u_{1} \frac{\partial u_{2}}{\partial v}\right) d S .
$$

Now it is easy to prove that if $\mathcal{C}_{q_{1}}=\mathcal{C}_{q_{2}}$ then the LHS of (17) is zero

$$
\int_{\Omega}\left(q_{1}-q_{2}\right) u_{1} u_{2} d x=0
$$

Now we extend $q_{i}=0$ in $\Omega^{c}$. We take solutions of $\left(\Delta-q_{i}\right) u_{i}=0$ in $\mathbb{R}^{n}$ of the form

$$
u_{i}=e^{x \cdot \rho_{i}}\left(1+\psi_{q_{i}}\left(x, \rho_{i}\right)\right), \quad i=1,2
$$


with $\left|\rho_{i}\right|$ large, $i=1,2$, with

$$
\begin{aligned}
& \rho_{1}=\frac{\eta}{2}+i\left(\frac{k+l}{2}\right) \\
& \rho_{2}=-\frac{\eta}{2}+i\left(\frac{k-l}{2}\right)
\end{aligned}
$$

and $\eta, k, l \in \mathbb{R}^{n}$ such that

$$
\begin{aligned}
& \eta \cdot k=k \cdot l=\eta \cdot l=0 \\
& |\eta|^{2}=|k|^{2}+|l|^{2} .
\end{aligned}
$$

Condition (21) guarantees that $\rho_{i} \cdot \rho_{i}=0, i=1,2$. Substituting (19) into (18) we conclude

$$
\left(\widehat{q_{1}-q_{2}}\right)(-k)=-\int_{\Omega} e^{i x \cdot k}\left(q_{1}-q_{2}\right)\left(\psi_{q_{1}}+\psi_{q_{2}}+\psi_{q_{1}} \psi_{q_{2}}\right) d x
$$

Now $\left\|\psi_{q_{i}}\right\|_{L^{2}(\Omega)} \leq \frac{C}{\left|\rho_{i}\right|}$. Therefore by taking $|l| \rightarrow \infty$ we obtain

$$
\left.\chi_{\Omega} \widehat{\left(q_{1}-\right.} q_{2}\right)(k)=0 \quad \forall k \in \mathbb{R}^{n}
$$

concluding the proof. Theorem 1.5 has been extended to conductivities having $3 / 2$ derivatives in some sense in $[32,152]$. Uniqueness for conormal conductivies in $C^{1+\epsilon}$ was shown in [63]. Recently Haberman and Tataru in a very nice article [75] have extended the uniqueness result to $C^{1}$ conductivities or small in the $W^{1, \infty}$ norm. It is an open problem whether uniqueness holds in dimension $n \geq 3$ for Lipschitz or less regular conductivities. Theorem 1.6 was extended to potentials in $L^{n / 2}$ and small potentials in the Fefferman-Phong class in [41]. For conormal potentials with strong singularities so that the potential is not in $L^{n / 2}$, for instance almost a delta function of an hypersurface, uniqueness was shown in [63].

Similar problems for higher order operators were considered in [97,118].

\subsubsection{Non-linear conductivities}

We now give an extension of this result to conductivities that depend on the voltage. Let $\gamma(x, t)$ be a function with domain $\bar{\Omega} \times \mathbb{R}$. Let $\alpha$ be such that $0<\alpha<1$. We assume

$$
\begin{aligned}
& \gamma \in C^{1, \alpha}(\bar{\Omega} \times[-T, T]), \quad \forall T, \\
& \gamma(x, t)>0, \forall(x, t) \in \bar{\Omega} \times \mathbb{R} .
\end{aligned}
$$


Given $f \in C^{2, \alpha}(\partial \Omega)$, there exists a unique solution of the Dirichlet problem (see [59])

$$
\begin{aligned}
\nabla \cdot(\gamma(x, u) \nabla u) & =0 \text { in } \Omega, \\
\left.u\right|_{\partial \Omega} & =f .
\end{aligned}
$$

Then the Dirichlet to Neumann map is defined by

$$
\Lambda_{\gamma}(f)=\left.\left.\gamma(x, f)\right|_{\partial \Omega} \frac{\partial u}{\partial v}\right|_{\partial \Omega}
$$

where $u$ is a solution to (25). Sun [184] proved the following result.

Theorem 1.7 Let $n \geq 3$. Assume $\gamma_{i} \in C^{1,1}(\bar{\Omega} \times[-T, T]) \forall T>0, i=1,2$, and $\Lambda_{\gamma_{1}}=\Lambda_{\gamma_{2}}$. Then $\gamma_{1}(x, t)=\gamma_{2}(x, t)$ on $\bar{\Omega} \times \mathbb{R}$.

The main idea is to linearize the Dirichlet to Neumann map at constant boundary data equal to the parameter $t$ (then the solution of (25) is equal to $t$ ). Isakov [96] was the first to use a linearization technique to study an inverse parabolic problems associated to non-linear equations. The case of the Dirichlet to Neumann map associated to the Schrödinger equation with a non-linear potential was considered in [99] under some assumptions on the potential. We note that, in contrast to the linear case, one cannot reduce the study of the inverse problem of the conductivity equation (25) to the Schrödinger equation with a non-linear potential. The main technical lemma in the proof of Theorem 1.7 is

Lemma 1.8 Let $\gamma(x, t)$ be as in (23) and (24). Let $1<p<\infty, 0<\alpha<1$. Let us define

$$
\gamma^{t}(x)=\gamma(x, t)
$$

Then for any $f \in C^{2, \alpha}(\partial \Omega), t \in \mathbb{R}$

$$
\lim _{s \rightarrow 0}\left\|\frac{1}{s} \Lambda_{\gamma}(t+s f)-\Lambda_{\gamma^{t}}(f)\right\|_{W^{1-\frac{1}{p}, p}(\partial \Omega)}=0 .
$$

The proof of Theorem 1.7 follows immediately from the lemma. Namely (28) and the hypotheses $\Lambda_{\gamma_{1}}=\Lambda_{\gamma_{2}} \Rightarrow \Lambda_{\gamma_{1}^{t}}=\Lambda_{\gamma_{2}^{t}}$ for all $t \in \mathbb{R}$. Then using the linear result, Theorem 1.5, we conclude that $\gamma_{1}^{t}=\gamma_{2}^{t}$ proving the theorem. We remark that the reduction from the non-linear problem to the linear is also valid in the two dimensional case [98]. Using the result of Astala and Päivärinta [11], which is reviewed in Sect. 1.6, one can extend Theorem 1.5 to $L^{\infty}(\Omega)$ conductivities in the two dimensional case. There are several open questions when the conductivity also depends on $\nabla u$, see [185] for a survey of results and open problems in this direction. 


\subsubsection{Other applications}

We give a short list of other applications to inverse problems using the CGO solutions described above for the Schrödinger equation.

- Quantum Scattering. In dimension $n \geq 3$ and in the case of a compactly supported electric potential, uniqueness for the fixed energy scattering problem was proven in $[135,145,159]$. In the earlier paper [146] this was done for small potentials. For compactly supported potentials, knowledge of the scattering amplitude at fixed energy is equivalent to knowing the Dirichlet-to-Neumann map for the Schrödinger equation measured on the boundary of a large ball containing the support of the potential (see [200,202] for an account). Then Theorem 1.6 implies the result. Melrose [128] suggested a related proof that uses the density of products of scattering solutions. Applications of CGO solutions to the 3-body problem were given in [203].

- Optics. The DN map associated to the Helmholtz equation $-\Delta+k^{2} n(x)$ with an isotropic index of refraction $n$ determines uniquely a bounded index of refraction in dimension $n \geq 3$.

- Optical tomography in the diffusion approximation. In this case we have $\nabla$. $\mathcal{D}(x) \nabla u-\sigma_{a}(x) u-i \omega u=0$ in $\Omega$ where $u$ represents the density of photons, $\mathcal{D}$ the diffusion coefficient, and $\sigma_{a}$ the optical absorption. Using the result of [190] one can show in dimension three or higher that if $\omega \neq 0$ one can recover both $\mathcal{D}$ and $\sigma_{a}$ from the corresponding DN map. If $\omega=0$ then one can recover one of the two parameters.

- Electromagnetics. For Maxwell's equations the analog of the DN map is the admittance map that maps the tangential component of the electric field to the tangential component of the magnetic field [175]. The admittance map for isotropic Maxwell's equations determines uniquely the isotropic electric permittivity, magnetic permeability and conductivity [147]. This system can in fact be reduced to the Schrödinger equation $\Delta-Q$ with $Q$ an $8 \times 8$ system and $\Delta$ the Laplacian times the identity matrix [148].

- Elasticity. For the isotropic elasticity system the problem of determining the Lamé parameters from the analog of the DN map in this case which sends the displacement at the boundary to the traction of the boundary has been solved if the Lamé parameter $\mu$ is close to a constant $[55,142,143]$.

- Determination of Inclusions and Obstacles. The CGO solutions constructed in Theorem 1.3 have been applied to determine inclusions for Helmholtz equations in [84] and Maxwell's equations in [209] using the enclosure method [83,84].

- Coupled-Physics Inverse Problems. In these problems one tries to combine the best features of two type of waves, one with high contrast and the other with high resolution to find the electromagnetic, optical or elastic properties of a medium. This combination is done through some physical principle. Examples are Photoacoustic and Thermoacoustic Tomography, Ultrasound Modulated Optical Tomography, Ultrasound Modulated Electrical Impedance Tomography, Magnetic Resonance Elastography and Transient Elastography among others. See $[13,176]$ for a review of some of these inverse methods. CGO solutions have been used in these hybrid methods in [15-18,42,110]. Inverse transport see [14] for applications of 60 solutions to inverse 
problem for the transport equation. The case where the electrical measurements are made on an unknown boundary was considered in [111].

\subsubsection{Stability}

The arguments used in the proofs of Theorems 1.5, 1.6, 1.1 can be pushed further to prove the following stability estimates proven in [4].

Theorem 1.9 Let $n \geq 3$. Suppose that $s>\frac{n}{2}$ and that $\gamma_{1}$ and $\gamma_{2}$ are $C^{\infty}$ conductivities on $\bar{\Omega} \subseteq \mathbb{R}^{n}$ satisfying

i) $0<\frac{1}{E} \leq \gamma_{j} \leq E, j=1,2$.

ii) $\left\|\gamma_{j}\right\|_{H^{s+2}(\Omega)} \leq E, j=1,2$.

Then there exist $C=C(\Omega, E, n, s)$ and $0<\sigma<1(\sigma=\sigma(n, s))$ such that

$$
\left\|\gamma_{1}-\gamma_{2}\right\|_{L^{\infty}(\Omega)} \leq C\left(\left|\log \left\|\Lambda_{\gamma_{1}}-\Lambda_{\gamma_{2}}\right\|_{\frac{1}{2}, \frac{-1}{2}}\right|^{-\sigma}+\left\|\Lambda_{\gamma_{1}}-\Lambda_{\gamma_{2}}\right\|_{\frac{1}{2}, \frac{-1}{2}}\right)
$$

where \|\|$_{\frac{1}{2}, \frac{-1}{2}}$ denotes the operator norm as operators from $H^{\frac{1}{2}}(\partial \Omega)$ to $H^{-\frac{1}{2}}(\partial \Omega)$.

Notice that this logarithmic type stability estimates indicates that the problem is severely ill-posed. Mandache [127] has shown that this estimate is optimal up to the value of the exponent. There is the question of whether under some additional a-priori condition one can improve this logarithmic type stability estimate. Alessandrini and Vessella [7] have shown that this is indeed the case and one has a Lipschitz type stability estimate if the conductivity is piecewise constant with jumps on a finite number of domains. Rondi [160] has subsequently shown that the constant in the estimate grows exponentially with the number of domains. It is conjectured, and this is supported by numerical experiments, that the stability estimate should be "better" near the boundary and gets increasingly worse as one penetrated deeper into the domain (Theorem 1.2 shows that at the boundary we have Lipschitz type stability estimate.) This type of depth dependence stability estimate has been proved in [138] for the case of some electrical inclusions. For a recent review of stability issues in EIT see [6]. Theorem 1.9 is a consequence of Theorem 1.2 and the following result.

Theorem 1.10 Assume 0 is not a Dirichlet eigenvalue of $\Delta-q_{i}, i=1,2$. Let $s>\frac{n}{2}$, $n \geq 3$ and

$$
\left\|q_{j}\right\|_{H^{s}(\Omega)} \leq M
$$

Then there exists $C=C(\Omega, M, n, s)$ and $0<\sigma<1(\sigma=\sigma(n, s))$ such that

$$
\left\|q_{1}-q_{2}\right\|_{H^{-1}(\Omega)} \leq C\left(\left|\log \left\|\Lambda_{q_{1}}-\Lambda_{q_{2}}\right\|_{\frac{1}{2}, \frac{-1}{2}}\right|^{-\sigma}+\left\|\Lambda_{q_{1}}-\Lambda_{q_{2}}\right\|_{\frac{1}{2}, \frac{-1}{2}}\right) .
$$

It was shown in [140] for the acoustic equation that the stability improves with frequency. 


\subsubsection{Reconstruction}

The complex geometrical optics solutions of Theorems 1.5 and 1.6 were also used by A. Nachman [135] and R. Novikov [145] to give a reconstruction procedure of the conductivity from $\Lambda_{\gamma}$. As we have already noticed in Sect. 1.2 we can reconstruct the conductivity at the boundary and its normal derivative from the DN map. Therefore if we know $\Lambda_{\gamma}$ we can determine $\Lambda_{q}$. We will then show how to reconstruct $q$ from $\Lambda_{q}$. Once this is done, to find $\sqrt{\gamma}$, we solve the problem

$$
\begin{aligned}
\Delta u-q u & =0 \text { in } \Omega, \\
\left.u\right|_{\partial \Omega} & =\left.\sqrt{\gamma}\right|_{\partial \Omega} .
\end{aligned}
$$

Let $q_{1}=q, q_{2}=0$ in formula (17). Then we have

$$
\int_{\Omega} q u v d x=\left.\int_{\partial \Omega}\left(\Lambda_{q}-\Lambda_{0}\right)\left(\left.v\right|_{\partial \Omega}\right) u\right|_{\partial \Omega} d S
$$

where $u, v \in H^{1}(\Omega)$ solve $\Delta u-q u=0, \Delta v=0$ in $\Omega$. Here $\Lambda_{0}$ denotes the Dirichlet to Neumann map associated to the potential $q=0$. We choose $\rho_{i}, i=1,2$ as in (20). Take $v=e^{x \cdot \rho_{1}}, u:=u_{\rho}=e^{x \cdot \rho_{2}}\left(1+\psi_{q}\left(x, \rho_{2}\right)\right)$ as in Theorem 1.3. By taking $|l| \rightarrow \infty$ in (32) we conclude

$$
\widehat{q}(-k)=\left.\lim _{|l| \rightarrow \infty} \int_{\partial \Omega}\left(\Lambda_{q}-\Lambda_{0}\right)\left(\left.e^{x \cdot \rho_{1}}\right|_{\partial \Omega}\right) u_{\rho}\right|_{\partial \Omega} d S .
$$

So the problem is then to recover the boundary values of the solutions $u_{\rho}$ from $\Lambda_{q}$. The idea is to find $\left.u_{\rho}\right|_{\partial \Omega}$ by looking at the exterior problem. Namely by extending $q=0$ outside $\Omega, u_{\rho}$ solves

$$
\begin{aligned}
\Delta u_{\rho} & =0 \text { in } \mathbb{R}^{n}-\Omega \\
\left.\frac{\partial u_{\rho}}{\partial v}\right|_{\partial \Omega} & =\Lambda_{q}\left(\left.u_{\rho}\right|_{\partial \Omega}\right) .
\end{aligned}
$$

Also note that

$$
e^{-x \cdot \rho_{2}} u_{\rho}-1 \in L_{\delta}^{2}\left(\mathbb{R}^{n}\right)
$$

Let $\rho \in \mathbb{C}^{n}-0$ with $\rho \cdot \rho=0$. Let $G_{\rho}(x, y) \in \mathcal{D}^{\prime}\left(\mathbb{R}^{n} \times \mathbb{R}^{n}\right)$ denote the Schwartz kernel of the operator $\Delta_{\rho}^{-1}$. Then we have that

$$
g_{\rho}(x)=e^{x \cdot \rho} G_{\rho}(x)
$$

is a Green's kernel for $\Delta$, namely

$$
\Delta g_{\rho}=\delta_{0} .
$$


We write the solution of (33) and (34) in terms of single and double layer potentials using this Green's kernel. This is also called Faddeev Green's kernel [56] who considered it in the context of scattering theory. We define the single and double layer potentials

$$
\begin{aligned}
& S_{\rho} f(x)=\int_{\partial \Omega} g_{\rho}(x-y) f(y) d S_{y}, \quad x \in \mathbb{R}^{n}-\Omega, \\
& D_{\rho} f(x)=\int_{\partial \Omega} \frac{\partial g_{\rho}}{\partial v}(x-y) f(y) d S_{y}, \quad x \in \mathbb{R}^{n}-\Omega \\
& B_{\rho} f(x)=p \cdot v \cdot \int_{\partial \Omega} \frac{\partial g_{\rho}}{\partial v}(x-y) f(y) d S_{y}, \quad x \in \partial \Omega .
\end{aligned}
$$

Nachman showed that $f_{\rho}=\left.u_{\rho}\right|_{\partial \Omega}$ is a solution of the integral equation

$$
f_{\rho}=e^{x \cdot \rho}-\left(S_{\rho} \Lambda_{q}-B_{\rho}-\frac{1}{2} I\right) f_{\rho}
$$

Moreover (40) is an inhomogeneous integral equation of Fredholm type for $f_{\rho}$ and it has a unique solution in $H^{\frac{3}{2}}(\partial \Omega)$. The uniqueness of the homogeneous equation follows from the uniqueness of the CGO solutions in Theorem 1.6.

\subsection{The partial data problem}

In several applications in EIT one can only measure currents and voltages on part of the boundary. Substantial progress has been made recently on the problem of whether one can determine the conductivity in the interior by measuring the DN map on part of the boundary. We review here the articles $[49,107]$. The paper [35] used the method of Carleman estimates with a linear weight to prove that, roughly speaking, knowledge of the DN map in "half" of the boundary is enough to determine uniquely a $C^{2}$ conductivity. The regularity assumption on the conductivity was relaxed to $C^{1+\epsilon}, \epsilon>$ 0 in [108]. Stability estimates for the uniqueness result of [35] were given in [76]. Stability estimates for the magnetic Schrödinger operator with partial data in the setting of [35] can be found in [198]. The result [35] was substantially improved in [107]. The latter paper contains a global identifiability result where it is assumed that the DN map is measured on any open subset of the boundary of a strictly convex domain for all functions supported, roughly, on the complement. We state the theorem more precisely below. The key new ingredient is the construction of a larger class of CGO solutions than the ones considered in Sect. 1.4. Let $x_{0} \in \mathbf{R}^{n} \backslash \overline{\operatorname{ch}(\Omega)}$, where $\operatorname{ch}(\Omega)$ denotes the convex hull of $\Omega$. Define the front and the back faces of $\partial \Omega$ by

$$
F\left(x_{0}\right)=\left\{x \in \partial \Omega ;\left(x-x_{0}\right) \cdot v \leq 0\right\}, B\left(x_{0}\right)=\left\{x \in \partial \Omega ;\left(x-x_{0}\right) \cdot v>0\right\} .
$$

The main result of [107] is the following: 
Theorem 1.11 Let $n>2$. With $\Omega, x_{0}, F\left(x_{0}\right), B\left(x_{0}\right)$ defined as above, let $q_{1}, q_{2} \in$ $L^{\infty}(\Omega)$ be two potentials and assume that there exist open neighborhoods $\widetilde{F}, \widetilde{B} \subset \partial \Omega$ of $F\left(x_{0}\right)$ and $B\left(x_{0}\right) \cup\left\{x \in \partial \Omega ;\left(x-x_{0}\right) \cdot v=0\right\}$ respectively, such that

$$
\Lambda_{q_{1}} u=\Lambda_{q_{2}} u \text { in } \widetilde{F}, \text { for all } u \in H^{\frac{1}{2}}(\partial \Omega) \cap \mathscr{E}^{\prime}(\widetilde{B}) \text {. }
$$

Then $q_{1}=q_{2}$.

Here $\mathscr{E}^{\prime}(\widetilde{B})$ denotes the space of compactly supported distributions in $\widetilde{B}$. The proof of this result uses Carleman estimates for the Laplacian with limiting Carleman weights (LCW). The Carleman estimates allow one to construct, for large $\tau$, a larger class of CGO solutions for the Schrödinger equation than previously used. These have the form

$$
u=e^{\tau(\phi+i \psi)}(a+r),
$$

where $\nabla \phi \cdot \nabla \psi=0,|\nabla \phi|^{2}=|\nabla \psi|^{2}$ and $\phi$ is the LCW. Moreover $a$ is smooth and non-vanishing and $\|r\|_{L^{2}(\Omega)}=O\left(\frac{1}{\tau}\right),\|r\|_{H^{1}(\Omega)}=O(1)$. Examples of LCW are the linear phase $\phi(x)=x \cdot \omega, \omega \in S^{n-1}$, used previously, and the non-linear phase $\phi(x)=\ln \left|x-x_{0}\right|$, where $x_{0} \in \mathbf{R}^{n} \backslash \overline{\operatorname{ch}(\Omega)}$ which was used in [107]. Any conformal transformation of these would also be a LCW. Below we give a characterization of all the LCW in $\mathbb{R}^{n}, n>2$, see [52]. In two dimensions any harmonic function is a LCW [205]. The CGO solutions used in [107] are of the form

$$
u(x, \tau)=e^{\ln \left|x-x_{0}\right|+i d\left(\frac{x-x_{0}}{\left|x-x_{0}\right|}, \omega\right)}(a+r)
$$

where $x_{0}$ is a point outside the convex hull of $\Omega, \omega$ is a unit vector and $d\left(\frac{x-x_{0}}{\left|x-x_{0}\right|}, \omega\right)$ denote distance. We take directions $\omega$ so that the distance function is smooth for $x \in \bar{\Omega}$.

\subsubsection{Limiting Carleman weights}

We only recall here the main ideas in the construction of the CGO solutions. We will denote $\tau=\frac{1}{h}$ in order to use the standard semiclassical notation. Let $P_{0}=-h^{2} \Delta$, where $h>0$ is a small semi-classical parameter. The weighted $L^{2}$-estimate

$$
\left\|e^{\phi / h} u\right\| \leq C\left\|e^{\phi / h} P_{0} u\right\|
$$

is of course equivalent to the unweighted estimate for a conjugated operator:

$$
\|v\| \leq C\left\|e^{\phi / h} P_{0} e^{-\phi / h} v\right\| .
$$

The semi-classical principal symbol of $P_{0}$ is $p(x, \xi)=\xi^{2}$, and that of the conjugated operator $e^{\phi / h} P_{0} e^{-\phi / h}$ is

$$
p\left(x, \xi+i \phi^{\prime}(x)\right)=a(x, \xi)+i b(x, \xi)
$$


where

$$
a(x, \xi)=\xi^{2}-\phi^{\prime}(x)^{2}, b(x, \xi)=2 \xi \cdot \phi^{\prime}(x) .
$$

Here we denote by $\phi^{\prime}$ the gradient of $\phi$. Write the conjugated operator as $A+i B$, with $A$ and $B$ formally selfadjoint and with $a$ and $b$ as their associated principal symbols. Then

$$
\|(A+i B) u\|^{2}=\|A u\|^{2}+\|B u\|^{2}+(i[A, B] u \mid u) .
$$

The principal symbol of $i[A, B]$ is $h\{a, b\}$, where $\{\cdot, \cdot\}$ denotes the Poisson bracket. In order to get enough negativity to satisfy Hörmander's solvability condition we require that

$$
a(x, \xi)=b(x, \xi)=0 \Rightarrow\{a, b\} \leq 0 .
$$

It is then indeed possible to get an a-priori estimate for the conjugated operator. We are led to the limiting case since we need to have CGO solutions for both $\phi$ and $-\phi$.

Definition 1.12 $\phi$ is a limiting Carleman weight (LCW) on some open set $\Omega$ if $\nabla \phi(x)$ is non-vanishing there and we have

$$
a(x, \xi)=b(x, \xi)=0 \Rightarrow\{a, b\}(x)=0, x \in \Omega .
$$

We remark that if $\phi$ is a LCW so is $-\phi$. In [52] we have classified locally all the LCW in Euclidean space.

Theorem 1.13 Let $\Omega$ be an open subset of $\mathbb{R}^{n}, n \geq 3$. The limiting Carleman weights in $\Omega$ are locally of the form

$$
\phi(x)=a \phi_{0}\left(x-x_{0}\right)+b
$$

where $a \in \mathbb{R} \backslash\{0\}$ and $\phi_{0}$ is one of the following functions:

$$
\begin{aligned}
&\langle x, \xi\rangle, \arg \left\langle x, \omega_{1}+i \omega_{2}\right\rangle, \\
& \log |x|, \quad \frac{\langle x, \xi\rangle}{|x|^{2}}, \quad \arg \left(e^{i \theta}(x+i \xi)^{2}\right), \quad \log \frac{|x+\xi|^{2}}{|x-\xi|^{2}}
\end{aligned}
$$

with $\omega_{1}, \omega_{2}$ orthogonal unit vectors, $\theta \in[0,2 \pi)$ and $\xi \in \mathbb{R}^{n} \backslash\{0\}$.

As noted earlier, in two dimensions, any harmonic function with a non-vanishing gradient is a limiting Carleman weight. 


\subsubsection{Construction of CGO Solutions with a non-linear phase}

A key ingredient in the construction of a richer family of CGO solutions is the following Carleman estimate.

Proposition 1.14 Let $\phi \in C^{\infty}(\operatorname{neigh}(\bar{\Omega}))$ be an LCW, $P=-h^{2} \Delta+h^{2} q, q \in$ $L^{\infty}(\Omega)$. Then, for $u \in C^{\infty}(\bar{\Omega})$, with $u_{\left.\right|_{\partial \Omega}}=0$, we have

$$
\begin{aligned}
& -\frac{h^{3}}{C}\left(\left(\phi_{x}^{\prime} \cdot v\right) e^{\phi / h} \partial_{\nu} u \mid e^{\phi / h} \partial_{\nu} u\right)_{\partial \Omega_{-}}+\frac{h^{2}}{C}\left(\left\|e^{\phi / h} u\right\|^{2}+\left\|e^{\phi / h} h \nabla u\right\|^{2}\right) \\
& \quad \leq C h^{3}\left(\left(\phi_{x}^{\prime} \cdot v\right) e^{\phi / h} \partial_{\nu} u \mid e^{\phi / h} \partial_{\nu} u\right)_{\partial \Omega_{+}}+\left\|e^{\phi / h} P u\right\|^{2},
\end{aligned}
$$

where norms and scalar products are in $L^{2}(\Omega)$ unless a subscript A (like for instance $\left.A=\partial \Omega_{-}\right)$indicates that they should be taken in $L^{2}(A)$. Here

$$
\partial \Omega_{ \pm}=\left\{x \in \partial \Omega ; \pm v(x) \cdot \phi^{\prime}(x) \geq 0\right\} .
$$

The proof of existence of solutions of the form (42) follows by using the HahnBanach theorem for the adjoint equation $e^{-\phi / h} P e^{\phi / h} u=v$. Let $\phi$ be a LCW and write $p\left(x, \xi+\phi^{\prime}(x)\right)=a(x, \xi)+i b(x, \xi)$. Then we know that $a$ and $b$ are in involution on their common zero set, and in this case it is well-known and exploited in [53] that we can find plenty of local solutions to the Hamilton-Jacobi system

$$
a\left(x, \psi^{\prime}(x)\right)=0, b\left(x, \psi^{\prime}(x)\right)=0 \Leftrightarrow \psi^{\prime 2}=\phi^{\prime 2}, \psi^{\prime} \cdot \phi^{\prime}=0
$$

We need the following more global statement:

Proposition 1.15 Let $\phi \in C^{\infty}($ neigh $(\bar{\Omega}))$ be a $L C W$, where $\Omega$ is a domain in $\mathbb{R}^{n}$ and define the hypersurface $G=p^{-1}\left(C_{0}\right)$ for some fixed value of $C_{0}$. Assume that each integral curve of $\phi^{\prime} \cdot \nabla_{x}$ through a point in $\Omega$ also intersects $G$ and that the corresponding projection map $\Omega \rightarrow G$ is proper. Then we get a solution of (45) in $C^{\infty}(\Omega)$ by solving first $g^{\prime}(x)^{2}=\phi^{\prime}(x)^{2}$ on $G$ and then defining $\psi$ by $\psi_{\left.\right|_{G}}=g$, $\phi^{\prime}(x) \cdot \partial_{x} \psi=0$. The vector fields $\phi^{\prime} \cdot \partial_{x}$ and $\psi^{\prime} \cdot \partial_{x}$ commute.

This result will be applied with a new domain $\Omega$ that contains the original one.

Next consider the WKB-problem

$$
P_{0}\left(e^{\frac{1}{h}(-\phi+i \psi)} a(x)\right)=e^{\frac{1}{h}(-\phi+i \psi)} \mathcal{O}\left(h^{2}\right) .
$$

The transport equation for $a$ is of Cauchy-Riemann type along the two-dimensional integral leaves of $\left\{\phi^{\prime} \cdot \partial_{x}, \psi^{\prime} \cdot \partial_{x}\right\}$. We have solutions that are smooth and everywhere $\neq 0$. (See [53]). The existence result for $e^{\phi / h} P e^{-\phi / h}$ mentioned in one of the remarks after Proposition 1.14 permits us to replace the right hand side of (46) by zero; more precisely, we can find $r=\mathcal{O}(h)$ in the semi-classical Sobolev space $H^{1}$ equipped with the norm $\|r\|=\|\langle h D\rangle r\|$, such that

$$
P\left(e^{\frac{1}{h}(-\phi+i \psi)}(a+r)\right)=0 .
$$




\subsubsection{The uniqueness proof}

We sketch the proof for the case that $\widetilde{B}=\partial \Omega$. All the arguments in this section are in dimension $n>2$. The arguments are similar to those of [35] using the new CGO solutions. Let $\phi$ be an LCW for which the constructions of Sect. 1.5.2 are available. Let $q_{1}, q_{2} \in L^{\infty}(\Omega)$ be as in Theorem 1.11 with

$$
\Lambda_{q_{1}}(f)=\Lambda_{q_{2}}(f) \quad \text { in } \partial \Omega_{-, \epsilon_{0}}, \forall f \in H^{\frac{1}{2}}(\partial \Omega),
$$

where

$$
\begin{aligned}
& \partial \Omega_{-, \epsilon_{0}}=\left\{x \in \partial \Omega ; \nu(x) \cdot \phi^{\prime}(x)<\epsilon_{0}\right\} \\
& \partial \Omega_{+, \epsilon_{0}}=\left\{x \in \partial \Omega ; \nu(x) \cdot \phi^{\prime}(x) \geq \epsilon_{0}\right\} .
\end{aligned}
$$

Let

$$
u_{2}=e^{\frac{1}{h}\left(\phi+i \psi_{2}\right)}\left(a_{2}+r_{2}\right)
$$

solve

$$
\left(\Delta-q_{2}\right) u_{2}=0 \text { in } \Omega,
$$

with $\left\|r_{2}\right\|_{H^{1}}=\mathcal{O}(h)$. Let $u_{1}$ solve

$$
\left(\Delta-q_{1}\right) u_{1}=0 \quad \text { in } \Omega, u_{\left.1\right|_{\partial \Omega}}=u_{\left.2\right|_{\partial \Omega}} .
$$

Then according to the assumptions in the theorem, we have $\partial_{\nu} u_{1}=\partial_{\nu} u_{2}$ in $\partial \Omega_{-, \epsilon_{0}}$ if $\epsilon_{0}>0$ has been fixed sufficiently small and we choose $\phi(x)=\ln \left|x-x_{0}\right|$.

Put $u=u_{1}-u_{2}, q=q_{2}-q_{1}$, so that

$$
\left(\Delta-q_{1}\right) u=q u_{2}, u_{\left.\right|_{\partial \Omega}}=0, \operatorname{supp}\left(\partial_{\nu} u_{\left.\right|_{\partial \Omega}}\right) \subset \partial \Omega_{+, \epsilon_{0}} .
$$

For $v \in H^{1}(\Omega)$ with $\Delta v \in L^{2}$, we get from Green's formula

$$
\begin{aligned}
\int_{\Omega} q u_{2} \bar{v} d x & =\int_{\Omega}\left(\Delta-q_{1}\right) u \bar{v} d x \\
& =\int_{\Omega} u \overline{\left(\Delta-\bar{q}_{1}\right) v} d x+\int_{\partial \Omega_{+, \epsilon_{0}}} \partial_{\nu} u \bar{v} d S .
\end{aligned}
$$

Similarly, we choose

$$
v=e^{-\frac{1}{h}\left(\phi+i \psi_{1}\right)}\left(a_{1}+r_{1}\right)
$$

with

$$
\left(\Delta-\bar{q}_{1}\right) v=0
$$


Then

$$
\int_{\Omega} q e^{\frac{i}{h}\left(\psi_{1}+\psi_{2}\right)}\left(a_{2}+r_{2}\right)\left(\overline{a_{1}+r_{1}}\right) d x=\int_{\partial \Omega_{+, \epsilon_{0}}} \partial_{\nu} u e^{-\frac{1}{h}\left(\phi-i \psi_{1}\right)}\left(\overline{a_{1}+r_{1}}\right) d S .
$$

Assume that $\psi_{1}, \psi_{2}$ are slightly $h$-dependent with

$$
\frac{1}{h}\left(\psi_{1}+\psi_{2}\right) \rightarrow f, h \rightarrow 0
$$

The left hand side of (51) tends to

$$
\int_{\Omega} q e^{i f} a_{2} \bar{a}_{1} d x
$$

when $h \rightarrow 0$. The modulus of the right hand side is

$$
\leq\left\|a_{1}+r_{1}\right\|_{L^{2}\left(\partial \Omega_{+, \epsilon_{0}}\right)}\left(\int_{\partial \Omega_{+, \epsilon_{0}}} e^{-2 \phi / h}\left|\partial_{\nu} u\right|^{2} d S\right)^{\frac{1}{2}}
$$

Here the first factor is bounded when $h \rightarrow 0$. In the Carleman estimate (44) we can replace $\phi$ by $-\phi$ and make the corresponding permutation of $\partial \Omega_{-}$and $\partial \Omega_{+}$. Applying this variant to the equation (49), we see that the second factor tends to 0 , when $h \rightarrow 0$. Thus,

$$
\int_{\Omega} e^{i f(x)} a_{2}(x) \overline{a_{1}(x)} q(x) d x=0 .
$$

Here we can arrange it so that $f, a_{2}, a_{1}$ are real-analytic and so that $a_{1}, a_{2}$ are nonvanishing. Moreover if $f$ can be attained as a limit of $\left(\psi_{1}+\psi_{2}\right) / h$ when $h \rightarrow 0$, so can $\lambda f$ for any $\lambda>0$. Thus we get the conclusion

$$
\int_{\Omega} e^{i \lambda f(x)} a_{2}(x) \overline{a_{1}(x)} q(x) d x=0 .
$$

To show that $q=0$ one uses arguments of analytic microlocal analysis [107]. A reconstruction method based on the uniqueness proof has been proposed in [137]. In [9] it was shown that if the potential is known in a neighborhood of the boundary and the DN map is measured on any open subset with Dirichlet data supported in the same set, the potential can be reconstructed from this data. It is an open problem whether this is valid in the general case. Isakov [95] proved a uniqueness result in dimension three or higher when the DN map is given on an arbitrary part of the boundary assuming that the remaining part is an open subset of a plane or a sphere and the DN map 
is measured on the plane or sphere. The case of partial data on a slab was studied in [122]. The DN map with partial data for the magnetic Schrödinger operator was studied in $[51,109,117,198]$. The case of the polyharmonic operator was considered in [118]. The case of Helmholtz equation [139]. We also mention that in [66] (resp. [101]) CGO approximate solutions are concentrated near planes (resp. spheres) and provided some local results related to the local DN map. It would be very interesting to extend the partial data result to systems. See [164] for Dirac systems, [40] for Maxwell and [91] for elasticity. Using methods of hyperbolic geometry similar to [101] it is shown in [82] that one can reconstruct inclusions from the local DN map using CGO solutions that decay exponentially inside a ball and grow exponentially outside. These are called complex spherical waves. A numerical implementation of this method has been done in [82]. The construction of complex spherical waves can also be done using the CGO solutions constructed in [107]. This was done in [204] in order to detect elastic inclusions, in [205] to detect inclusions in the two dimensional case for a large class of systems with inhomogeneous background, and in [165] for the case of inclusions contained in a slab. Partial data for higher order elliptic operators has been studied in [119]. We mention that methods of hyperbolic geometry have been also studied earlier in the works $[19,58,166]$.

\subsubsection{The Linearized Calderón partial data problem}

It is an open problem in dimension $n \geq 3$ that if the Dirichlet to Neumann map for the conductivity or potential is measured on an open non-empty subset of the boundary for Dirichlet data supported in that set we can determine uniquely the potential. In this section we consider the linearized version of this problem, generalizing Calderón's approach. We add the constraint that the restriction of the harmonic functions to the boundary vanishes on any fixed closed proper subset of the boundary. We show that the product of these harmonic functions is dense. More precisely

Theorem 1.16 Let $\Omega$ be a connected bounded open set in $\mathbb{R}^{n}, n \geq 2$, with smooth boundary. The set of products of harmonic functions on $\Omega$ which vanish on a closed proper subset $\Gamma \subsetneq \partial \Omega$ of the boundary is dense in $L^{1}(\Omega)$.

Sketch of the Proof. We take $f \in L^{1}(\Omega)$. Assume

$$
\int_{\Omega} f u v d x=0,
$$

for all harmonic functions $u, v$ with $\left.u\right|_{\Gamma}=\left.v\right|_{\Gamma}=0$. First one proves a local result. Fix a point $x_{0}$ on the boundary. It is shown that if $f=0$ in a neighborhood of $x_{0}$ then $f=0$ in the whole domain. See [49] for the proof. We now extend $f=0$ outside $\Omega$. We reduce the problem to the case where the point $x_{0}$ has a hyperplane tangent to the boundary at the point $x_{0}$. We use Calderón's exponential solutions for all the possible complex frequencies $\rho$ such that $\rho \cdot \rho=0$ (previously we used in Sects. 1.4 and 1.5.3 the cancellation of the real parts when taking the products). Using these solutions and (53) one obtains a decay of the Bargmann-Segal transform of $f$ (see [173]) 


$$
T f(z)=\int_{\mathbb{R}^{n}} e^{-\frac{1}{2 h}(z-y)^{2} f(y) d y}
$$

for certain complex directions. Using the watermelon approach [105,174], one then shows that there is an exponential decay of this transform for other directions implying that the point $\left(x_{0}, v\right)$, where $v$ is the normal to the point $x_{0}$, is not in the analytic wave front set of $f$ in contradiction to the microlocal version of Holmgren's uniqueness theorem $[81,173]$. We explain below some of the details of the proof. One can assume that $\Omega \backslash\left\{x_{0}\right\}$ is on one side of the tangent hyperplane $T_{x_{0}}(\Omega)$ at $x_{0}$ by making a conformal transformation. Pick $a \in \mathbb{R}^{n} \backslash \bar{\Omega}$ which sits on the line segment in the direction of the outward normal to $\partial \Omega$ at $x_{0}$; there is a ball $B(a, r)$ such that $\partial B(a, r) \cap \bar{\Omega}=\left\{x_{0}\right\}$, and there is a conformal transformation

$$
\psi: \mathbb{R}^{n} \backslash B(a, r) \rightarrow \overline{B(a, r)}
$$

which fixes $x_{0}$ and exchanges the interior and the exterior of the ball $B(a, r)$. The hyperplane $H:\left(x-x_{0}\right) \cdot\left(a-x_{0}\right)=0$ is tangent to $\psi(\Omega)$, and the image $\psi(\Omega) \backslash\left\{x_{0}\right\}$ by the conformal transformation lies inside the ball $B(a, r)$, therefore on one side of $H$. The fact that functions are supported on the boundary close to $x_{0}$ is left unchanged. Since a function is harmonic on $\Omega$ if and only if its Kelvin transform

$$
u^{*}=r^{n-2}|x-a|^{-n+2} u \circ \psi
$$

is harmonic on $\psi(\Omega),(1.53)$ becomes

$$
0=\int_{\Omega} f u v d x=\int_{\psi(\Omega)} r^{4}|x-a|^{-4} f \circ \psi u^{*} v^{*} d x
$$

for all harmonic functions $u^{*}, v^{*}$ on $\psi(\Omega)$. If one can prove that if $|x-a|^{-4} f \circ \psi$ vanishes close to $x_{0}$ then so does $f$. Moreover, by scaling one can assume that $\Omega$ is contained in a ball of radius 1 . Our setting will therefore be as follows: $x_{0}=0$, the tangent hyperplane at $x_{0}$ is given by $x_{1}=0$ and

$$
\Omega \subset\left\{x \in \mathbb{R}^{n}:\left|x+e_{1}\right|<1\right\}, \quad \Gamma=\left\{x \in \partial \Omega: x_{1} \geq-2 c\right\} .
$$

The prime will be used to denote the last $n-1$ variables so that $x=\left(x_{1}, x^{\prime}\right)$ for instance. The Laplacian on $\mathbb{R}^{n}$ has $p(\xi)=\xi^{2}$ as a principal symbol, if we still denote by $p(\zeta)=\zeta^{2}$ the continuation of this principal symbol on $\mathbb{C}^{n}$, we consider

$$
p^{-1}(0)=\left\{\zeta \in \mathbb{C}^{n}: \zeta^{2}=0\right\}
$$

In dimension $n=2$, this set is the union of two complex lines

$$
p^{-1}(0)=\mathbb{C} \gamma \cup \mathbb{C} \bar{\gamma}
$$


where $\gamma=i e_{1}+e_{2}=(i, 1) \in \mathbb{C}^{2}$. Note that $(\gamma, \bar{\gamma})$ is a basis of $\mathbb{C}^{2}:$ the decomposition of a complex vector in this basis reads

$$
\zeta=\zeta_{1} e_{1}+\zeta_{2} e_{2}=\frac{\zeta_{2}-i \zeta_{1}}{2} \gamma+\frac{\zeta_{2}+i \zeta_{1}}{2} \bar{\gamma}
$$

Similarly for $n \geq 2$, the differential of the map

$$
\begin{aligned}
s: p^{-1}(0) \times p^{-1}(0) & \rightarrow \mathbb{C}^{n} \\
(\zeta, \eta) & \mapsto \zeta+\eta
\end{aligned}
$$

at $\left(\zeta_{0}, \eta_{0}\right)$ is surjective

$$
\begin{aligned}
D s\left(\zeta_{0}, \eta_{0}\right): T_{\zeta_{0}} p^{-1}(0) \times T_{\eta_{0}} p^{-1} \quad(0) & \rightarrow \mathbb{C}^{n} \\
(\zeta, \eta) & \mapsto \zeta+\eta
\end{aligned}
$$

provided $\mathbb{C}^{n}=T_{\zeta_{0}} p^{-1}(0)+T_{\eta_{0}} p^{-1}(0)$, i.e. provided $\zeta_{0}$ and $\eta_{0}$ are linearly independent. In particular, this is the case if $\zeta_{0}=\gamma$ and $\eta_{0}=-\bar{\gamma}$; as a consequence all $z \in \mathbb{C}^{n},\left|z-2 i e_{1}\right|<2 \varepsilon$ may be decomposed as a sum of the form

$$
z=\zeta+\eta, \quad \text { with } \zeta, \eta \in p^{-1}(0),|\zeta-\gamma|<C \varepsilon,|\eta+\bar{\gamma}|<C \varepsilon
$$

provided $\varepsilon>0$ is small enough. The exponentials with linear weights

$$
e^{-\frac{i}{h} x \cdot \zeta}, \quad \zeta \in p^{-1}(0)
$$

are harmonic functions. We need to add a correction term in order to obtain harmonic functions $u$ satisfying the boundary requirement $\left.u\right|_{\Gamma}=0$. Let $\chi \in \mathcal{D}\left(\mathbb{R}^{n}\right)$ be a cutoff function which equals 1 on $\Gamma$, and consider the solution $w$ to the Dirichlet problem

$$
\Delta w=0 \quad \text { in } \Omega,\left.w\right|_{\partial \Omega}=-\left.\left(e^{-\frac{i}{h} x \cdot \zeta} \chi\right)\right|_{\partial \Omega} .
$$

The function

$$
u(x, \zeta)=e^{-\frac{i}{h} x \cdot \zeta}+w(x, \zeta)
$$

is harmonic and satisfies $\left.u\right|_{\Gamma}=0$. We have the following bound on $w$ :

$$
\begin{aligned}
\|w\|_{H^{1}(\Omega)} & \leq C_{1}\left\|e^{-\frac{i}{h} x \cdot \zeta} \chi\right\|_{H^{\frac{1}{2}}(\partial \Omega)} \\
& \leq C_{2}\left(1+h^{-1}|\zeta|\right)^{\frac{1}{2}} e^{\frac{1}{h} H_{K}(\operatorname{Im} \zeta)}
\end{aligned}
$$

where $H_{K}$ is the supporting function of the compact subset $K=\operatorname{supp} \chi \cap \partial \Omega$ of the boundary

$$
H_{K}(\xi)=\sup _{x \in K} x \cdot \xi, \quad \xi \in \mathbb{R}^{n}
$$


In particular, if we take $\chi$ to be supported in $x_{1} \leq-c$ and equal to 1 on $x_{1} \leq-2 c$ then the bound (60) becomes

$$
\|w\|_{H^{1}(\Omega)} \leq C_{2}\left(1+h^{-1}|\zeta|\right)^{\frac{1}{2}} e^{-\frac{c}{h} \operatorname{Im} \zeta_{1}} e^{\frac{1}{h}\left|\operatorname{Im} \zeta^{\prime}\right|} \quad \text { when } \operatorname{Im} \zeta_{1} \geq 0
$$

The starting point is the cancellation of the integral

$$
\int_{\Omega} f(x) u(x, \zeta) u(x, \eta) d x=0, \quad \zeta, \eta \in p^{-1}(0)
$$

which may be rewritten in the form

$$
\begin{aligned}
\int_{\Omega} f(x) e^{-\frac{i}{h} x \cdot(\zeta+\eta)} d x= & -\int_{\Omega} f(x) e^{-\frac{i}{h} x \cdot \zeta} w(x, \eta) d x \\
& -\int_{\Omega} f(x) e^{-\frac{i}{h} x \cdot \eta} w(x, \zeta) d x-\int_{\Omega} f(x) w(x, \zeta) w(x, \eta) d x .
\end{aligned}
$$

This allows to give a bound on the left-hand side

$$
\begin{aligned}
& \left|\int_{\Omega} f(x) e^{-\frac{i}{h} x \cdot(\zeta+\eta)} d x\right| \leq\|f\|_{L^{\infty}(\Omega)}\left(\left\|e^{-\frac{i}{h} x \cdot \zeta}\right\|_{L^{2}(\Omega)}\|w(x, \eta)\|_{L^{2}(\Omega)}\right. \\
& \left.\quad+\left\|e^{-\frac{i}{h} x \cdot \eta}\right\|_{L^{2}(\Omega)}\|w(x, \zeta)\|_{L^{2}(\Omega)}+\|w(x, \eta)\|_{L^{2}(\Omega)}\|w(x, \zeta)\|_{L^{2}(\Omega)}\right) .
\end{aligned}
$$

Thus using (61)

$$
\begin{aligned}
\left|\int_{\Omega} f(x) e^{-\frac{i}{h} x \cdot(\zeta+\eta)} d x\right| \leq & C_{3}\|f\|_{L^{\infty}(\Omega)}\left(1+h^{-1}|\eta|\right)^{\frac{1}{2}}\left(1+h^{-1}|\zeta|\right)^{\frac{1}{2}} \\
& \times e^{-\frac{c}{h} \min \left(\operatorname{Im} \zeta_{1}, \operatorname{Im} \eta_{1}\right)} e^{\frac{1}{h}\left(\left|\operatorname{Im} \zeta^{\prime}\right|+\left|\operatorname{Im} \eta^{\prime}\right|\right)}
\end{aligned}
$$

when $\operatorname{Im} \zeta_{1} \geq 0, \operatorname{Im} \eta_{1} \geq 0$ and $\zeta, \eta \in p^{-1}(0)$. In particular, if $|\zeta-a \gamma|<C \varepsilon a$ and $|\eta+a \bar{\gamma}|<C \varepsilon a$ with $\varepsilon \leq 1 / 2 C$ then

$$
\left|\int_{\Omega} f(x) e^{-\frac{i}{h} x \cdot(\zeta+\eta)} d x\right| \leq C_{4} h^{-1}\|f\|_{L^{\infty}(\Omega)} e^{-\frac{c a}{2 h}} e^{\frac{2 C \varepsilon a}{h}} .
$$

Take $z \in \mathbb{C}^{n}$ with $\left|z-2 a e_{1}\right|<2 \varepsilon a$ and with $\varepsilon$ small enough. Once rescaled the decomposition (58) gives

$$
z=\zeta+\eta, \quad \zeta, \eta \in p^{-1}(0),|\zeta-a \gamma|<C \varepsilon a,|\eta+a \bar{\gamma}|<C \varepsilon a
$$


and we therefore get the estimate

$$
\left|\int_{\Omega} f(x) e^{-\frac{i}{h} x \cdot z} d x\right| \leq C_{4} h^{-1}\|f\|_{L^{\infty}(\Omega)} e^{-\frac{c a}{2 h}} e^{\frac{2 C \varepsilon a}{h}}
$$

for all $z \in \mathbb{C}^{n}$ such that $\left|z-2 a i e_{1}\right|<2 \varepsilon a$. This implies that the Bargmann-Segal transform of $f$ satisfies

$$
|T f(z)| \leq C \mid f \|_{L^{\infty}(\Omega)} e^{\frac{1}{2 h}\left(|\operatorname{Im} z|^{2}-|\operatorname{Re} z|^{2}-\frac{c a}{2}\right)}
$$

for some $\epsilon, a, c>0$ and for all $z \in \mathbb{C}^{n}$ such that $\left|z-2 a i e_{1}\right|<2 \varepsilon a$. By the definition of the analytic wave front set, the last estimate says that the point $\left(0,2 a e_{1}\right)$ is not in the analytic wave front set of $f$. By Kashiwara's watermelon theorem [105, 174], since $f$ is supported in the half space $x_{1} \leq 0$, if 0 is in the support of $f$ then $(0, v)$ with $v$ the unit normal to the boundary is also in the analytic wave front set but this is a contradiction since $2 a e_{1}$ is also normal to $x_{1}=0$. Therefore 0 is not in the support of $f$ and $f$ vanishes in a neighborhood of 0 .

\subsection{The Calderón problem in two dimensions}

Astala and Päivärinta [11], in a seminal contribution, have recently extended significantly the uniqueness result of [134] for conductivities having two derivatives in an appropriate sense and the result of [33] for conductivities having one derivative in appropriate sense, by proving that any $L^{\infty}$ conductivity in two dimensions can be determined uniquely from the DN map. We remark that the method of [33,134] uses, besides CGO solutions, the $\bar{\partial}$ method introduced in one dimension by Beals and Coifman [21] and generalized to several dimensions in $[1,22,136,196]$. The $\bar{\partial}$ method has been used in numerical reconstruction procedures in two dimensions in $[93,172]$ among others. The proof of [11] relies also on construction of CGO solutions for the conductivity equation with $L^{\infty}$ coefficients and the $\bar{\partial}$ method. This is done by transforming the conductivity equation to a quasi-regular map. Let $\mathcal{D}$ be the unit disk in the plane. Then we have

Lemma 1.17 Assume $u \in H^{1}(\mathcal{D})$ is real valued and satisfies the conductivity equation on $\mathcal{D}$. Then there exists a function $v \in H^{1}(\mathcal{D})$, unique up to a constant, such that $f=u+i v$ satisfies the Beltrami equation

$$
\bar{\partial} f=\mu \overline{\partial f},
$$

where $\mu=(1-\gamma) /(1+\gamma)$. Conversely, if $f \in H^{1}(\mathcal{D})$ satisfies $(65)$ with a real-valued $\mu$, then $u=\operatorname{Re} f$ and $v=\operatorname{Im} f$ satisfy

$$
\nabla \cdot \gamma \nabla u=0 \text { and } \nabla \cdot \frac{1}{\gamma} \nabla v=0,
$$

respectively, where $\gamma=(1-\mu) /(1+\mu)$. 
Let us denote $\kappa=\|\mu\|_{L^{\infty}}<1$. Then (65) means that $f$ is a quasi-regular map. The function $v$ is called the $\gamma$-harmonic conjugate of $u$ and it is unique up to a constant. Astala and Päivärinta consider the $\mu$-Hilbert transform $\mathcal{H}_{\mu}: H^{1 / 2}(\partial \Omega) \rightarrow H^{1 / 2}(\partial \Omega)$ that is defined by

$$
\mathcal{H}_{\mu}:\left.\left.u\right|_{\partial \Omega} \mapsto v\right|_{\partial \Omega}
$$

and show that the DN map $\Lambda_{\gamma}$ determines $\mathcal{H}_{\mu}$ and vice versa. Below we use the complex notation $z=x_{1}+i x_{2}$. Moreover, for the Eq. (65), it is shown that for every $k \in \mathbb{C}$ there are complex geometrical optics solutions of the Beltrami equation that have the form

$$
f_{\mu}(z, k)=e^{i k z} M_{\mu}(z, k)
$$

where

$$
M_{\mu}(z, k)=1+\mathcal{O}\left(\frac{1}{z}\right) \text { as }|z| \rightarrow \infty .
$$

In the case of non-smooth coefficients the function $M_{\mu}$ grows sub-exponentially in $k$. Astala and Päivärinta introduce the "transport matrix" to deal with this problem. Using a result of Bers connecting pseudoanalytic functions with quasi-regular maps they show that this matrix is determined by the Hilbert transform $H_{\mu}$ and therefore the DN map. Then they use the transport matrix to show that $\Lambda_{\gamma}$ determines uniquely $\gamma$. See [11] for more details. Logarithmic type stability estimates for Hölder conductivities of positive exponent have been given in [20].

\subsubsection{Bukhgeim's result}

In a recent breakthrough, Bukhgeim [34] proved that a potential in $W^{2, p}(\Omega), p>2$ can be uniquely determined from the set of Cauchy data as defined in (15). An earlier result [187] gave this for a generic class of potentials. As before, if two potentials $q_{1}, q_{2}$ have the same set of Cauchy data, we have

$$
\int_{\Omega}\left(q_{1}-q_{2}\right) u_{1} u_{2} d x=0
$$

where $u_{i}, i=1,2$, are solutions of the Schrödinger equation. Assume now that $0 \in \Omega$. Bukhgeim takes CGO solutions of the form

$$
u_{1}(z, k)=e^{z^{2} k}\left(1+\psi_{1}(z, k)\right), \quad u_{2}(z, k)=e^{-\bar{z}^{2} k}\left(1+\psi_{2}(z, k)\right)
$$

where $z, k \in \mathbb{C}$ and we have used the complex notation $z=x_{1}+i x_{2}$. Moreover $\psi_{1}$ and $\psi_{2}$ decay uniformly in $\Omega$, in an appropriate sense, for $|k|$ large. Note that the weight $z^{2} k$ in the exponential is a limiting Carleman weight since it is a harmonic function 
but it is singular at 0 since its gradient vanishes there. Substituting (70) into (69) we obtain

$$
\int_{\Omega} e^{2 i \tau x_{1} x_{2}}\left(q_{1}-q_{2}\right)\left(1+\psi_{1}+\psi_{2}+\psi_{1} \psi_{2}\right) d x=0
$$

Now using the decay of $\psi_{i}$ in $\tau, i=1,2$, and applying stationary phase (the phase function $x_{1} x_{2}$ that has a non-degenerate critical point at 0 ) we obtain $q_{1}(0)=q_{2}(0)=$ 0 in $\Omega$. Of course we can do this at any point of $\Omega$ proving the result. This result also shows that complex conductivities can be determined uniquely from the DN map. Francini has shown in [57] that this was the case for conductivities with small imaginary part. It also implies unique determination of a potential from the fixed energy scattering amplitude in two dimensions. A general result for first order systems is in [3], generalizing the results of [71] and [104].

\subsubsection{Partial data problem in $2 D$}

It is shown in [87] that for a two dimensional bounded domain the Cauchy data for the Schrödinger equation measured on an arbitrary open subset of the boundary determines uniquely the potential. This implies, for the conductivity equation, that if one measures the current fluxes at the boundary on an arbitrary open subset of the boundary produced by voltage potentials supported in the same subset, one can determine uniquely the conductivity. The paper [87] uses Carleman estimates with weights which are harmonic functions with non-degenerate critical points to construct appropriate complex geometrical optics solutions to prove the result. We describe this more precisely below. Let $\Omega \subset \mathbb{R}^{2}$ be a bounded domain which consists of $N$ smooth closed curves $\gamma_{j}, \partial \Omega=\cup_{j=}^{N} \gamma_{j}$. As before we define the set of Cauchy data for a bounded potential $q$ by:

$$
\widehat{C_{q}}=\left\{\left(\left.u\right|_{\partial \Omega},\left.\frac{\partial u}{\partial v}\right|_{\partial \Omega}\right) \mid(\Delta-q) u=0 \text { on } \Omega, u \in H^{1}(\Omega)\right\} .
$$

Let $\Gamma \subset \partial \Omega$ be a non-empty open subset of the boundary. Denote $\Gamma_{0}=\partial \Omega \backslash \bar{\Gamma}$. The main result of [87] gives global uniqueness by measuring the Cauchy data on $\Gamma$. Let $q_{j} \in C^{2+\alpha}(\bar{\Omega}), j=1,2$ for some $\alpha>0$ and let $q_{j}$ be complex-valued. Consider the following sets of Cauchy data on $\Gamma$ :

$$
\mathcal{C}_{q_{j}}=\left\{\left(\left.u\right|_{\Gamma},\left.\frac{\partial u}{\partial v}\right|_{\Gamma}\right)\left|\left(\Delta-q_{j}\right) u=0 \operatorname{in} \Omega, u\right|_{\Gamma_{0}}=0, u \in H^{1}(\Omega)\right\}, \quad j=1,2 .
$$

Theorem 1.18 Assume $\mathcal{C}_{q_{1}}=\mathcal{C}_{q_{2}}$. Then $q_{1}=q_{2}$.

Using Theorem 1.18 one concludes immediately, as a corollary, the following global identifiability result for the conductivity Eq. (2). This result uses that knowledge of 
the Dirichlet-to-Neumann map on an open subset of the boundary determines $\gamma$ and its first derivatives on $\Gamma$ (see $[113,192])$.

Corollary 1.19 With some $\alpha>0$, let $\gamma_{j} \in C^{4+\alpha}(\bar{\Omega}), j=1,2$, be non-vanishing functions. Assume that

$$
\Lambda_{\gamma_{1}}(f)=\Lambda_{\gamma_{2}}(f) \text { on } \Gamma \text { for all } f \in H^{\frac{1}{2}}(\Gamma) \text {, supp } f \subset \Gamma \text {. }
$$

Then $\gamma_{1}=\gamma_{2}$.

It is easy to see that Theorem 1.18 implies the analogous result to [107] in the two dimensional case. Notice that Theorem 1.18 does not assume that $\Omega$ is simply connected. An interesting inverse problem is whether one can determine the potential or conductivity in a region of the plane with holes by measuring the Cauchy data only on the accessible boundary. This is also called the obstacle problem. Let $\Omega, D$ be domains in $\mathbb{R}^{2}$ with smooth boundaries such that $\bar{D} \subset \Omega$. Let $V \subset \partial \Omega$ be an open set. Let $q_{j} \in C^{2+\alpha}(\overline{\Omega \backslash D})$, for some $\alpha>0$ and $j=1,2$. Let us consider the following set of partial Cauchy data

$$
\widetilde{C}_{q_{j}}=\left\{\left(\left.u\right|_{V},\left.\frac{\partial u}{\partial v}\right|_{V}\right) \mid\left(\Delta-q_{j}\right) u=0 \text { in } \Omega \backslash \bar{D},\left.u\right|_{\partial D \cup \partial \Omega \backslash V}=0, u \in H^{1}(\Omega \backslash \bar{D})\right\} .
$$

Corollary 1.20 Assume $\widetilde{C}_{q_{1}}=\widetilde{C}_{q_{2}}$. Then $q_{1}=q_{2}$.

A similar result holds for the conductivity equation.

Corollary 1.21 Let $\gamma_{j} \in C^{4+\alpha}(\overline{\Omega \backslash D}) j=1,2$ be non vanishing functions. Assume

$$
\Lambda_{\gamma_{1}}(f)=\Lambda_{\gamma_{2}}(f) \text { on } V \quad \forall f \in H^{\frac{1}{2}}(\partial(\bar{\Omega} \backslash D)) \text {, supp } f \subset V
$$

Then $\gamma_{1}=\gamma_{2}$.

The two dimensional case has special features since one can construct a much larger set of complex geometrical optics solutions than in higher dimensions. On the other hand, the problem is formally determined in two dimensions and therefore more difficult. The proof of Theorem 1.18 is based on the construction of appropriate complex geometrical optics solutions by Carleman estimates with degenerate weight functions.

Sketch of the Proof. For the partial data problem we need a more general class of CGO solutions than the ones constructed by Bukhgeim, since we would like to have the imaginary part of the phase vanish on $\Gamma$. So we consider more general holomorphic functions with non-degenerate critical points as phases. Let the function $\Phi(z)=$ $\varphi\left(x_{1}, x_{2}\right)+i \psi\left(x_{1}, x_{2}\right) \in C^{2}(\bar{\Omega})$ be holomorphic in $\Omega$ and $\left.\operatorname{Im} \Phi\right|_{\partial \Omega \backslash \Gamma}=0$. Notice that this implies $\nabla \varphi \cdot v=0$ on $\partial \Omega \backslash \Gamma$. We denote the set of critical points of $\Phi$ by

$$
\mathcal{H}=\left\{z \in \bar{\Omega} \mid \partial_{z} \Phi(z)=0\right\}
$$


We assume that $\Phi$ has a finite number of non-degenerate critical points in $\bar{\Omega}$, that is $\partial_{z}^{2} \Phi(z) \neq 0, z \in \mathcal{H}$. We denote the critical points by $\mathcal{H}=\left\{\widetilde{x}_{1}, \ldots, \widetilde{x}_{\ell}\right\}$ As in the partial data problem considered in Sect. 1.5 we construct appropriate CGO solutions by proving a Carleman estimate.

Carleman estimate Let $u \in H_{0}^{1}(\Omega)$, real valued. Then for all large $\tau>0$ :

$$
\begin{aligned}
& \tau\left\|u e^{\tau \varphi}\right\|_{L^{2}(\Omega)}^{2}+\left\|u e^{\tau \varphi}\right\|_{H^{1}(\Omega)}^{2}+\left\|\frac{\partial u}{\partial \nu} e^{\tau \varphi}\right\|_{L^{2}(\partial \Omega \backslash \Gamma)}^{2}+\tau^{2}\left\|\left|\frac{\partial \Phi}{\partial z}\right| u e^{\tau \varphi}\right\|_{L^{2}(\Omega)}^{2} \\
& \leq C\left(\left\|(\Delta u) e^{\tau \varphi}\right\|_{L^{2}(\Omega)}^{2}+\tau \int_{\Gamma}\left|\frac{\partial u}{\partial v}\right|^{2} e^{2 \tau \varphi} d \sigma\right)
\end{aligned}
$$

with $\sigma$ the standard measure on $\partial \Omega$. The Carleman estimate implies the existence of a solution to the boundary value problem for the Schrödinger equation

$$
(\Delta-q) u=f \text { in } \Omega ;\left.\quad u\right|_{\partial \Omega \backslash \Gamma}=g
$$

and that it satisfies an estimate. More precisely we have

Proposition 1.22 Let $q \in L^{\infty}(\Omega)$. There exists $\tau_{0}>0$ such that for all $|\tau|>\tau_{0}$ there exists a solution of (72) such that

$$
\left\|u e^{-\tau \varphi}\right\|_{L^{2}(\Omega)} \leq C\left(\left\|f e^{-\tau \varphi}\right\|_{L^{2}(\Omega)} / \tau+\left\|g e^{-\tau \varphi}\right\|_{L^{2}(\partial \Omega \backslash \Gamma)}\right) .
$$

We next find CGO solutions of

$$
(\Delta-q) u=0 \text { in } \Omega ;\left.\quad u\right|_{\partial \Omega \backslash \widetilde{\Gamma}}=0
$$

of the form

$$
u(x)=e^{\tau \Phi(z)}\left(a(z)+a_{0}(z) / \tau\right)+e^{\tau \overline{\Phi(z)}} \overline{\left(a(z)+a_{1}(z) / \tau\right)}+e^{\tau \varphi} u_{1}+e^{\tau \varphi} u_{2} .
$$

The functions $a, a_{0}, a_{1} \in C^{2}(\bar{\Omega})$ are holomorphic in $\Omega$ and $\left.\operatorname{Re} a\right|_{\partial \Omega \backslash \Gamma}=0$. Moreover

$$
\left\|u_{j}\right\|_{L^{2}(\Omega)}=o\left(\frac{1}{\tau}\right), \quad \tau \rightarrow \infty, j=1,2 .
$$

Now we take two potentials $q_{1}$ and $q_{2}$ satisfying the hypothesis of Theorem 1.18 . We take for the potential $q_{1}$ a solution $u$ of the corresponding Schrödinger equation of the form (75) and for the Schrödinger equation associated to $q_{2}$ a solution $v$ of the form

$$
v(x)=e^{-\tau \Phi(z)}\left(a(z)+b_{0}(z) / \tau\right)+e^{-\tau \overline{\Phi(z)}} \overline{\left(a(z)+b_{1}(z) / \tau\right)}+e^{-\tau \varphi} v_{1}+e^{-\tau \varphi} v_{2}
$$


with $v_{1}, v_{2}$ satisfying the same decay for large $\tau$ as $u_{1}, u_{2}$. Using arguments similar to those of Sect. 1.4.1 we get

$$
\int_{\Omega}\left(q_{1}-q_{2}\right) u v d x=0
$$

Substituting (74) and (77) into this identity and applying stationary phase we conclude

Proposition 1.23 Let $\left\{\widetilde{x}_{1}, \ldots, \tilde{x}_{\ell}\right\}$ be the set of critical points of the function Im $\Phi$. Then for any potentials $q_{1}, q_{2}$ satisfying the hypotheses of Theorem 1.18 and for any holomorphic function $a$, we have

$$
2 \sum_{k=1}^{\ell} \frac{\pi\left(\left(q_{1}-q_{2}\right)|a|^{2}\right)\left(\widetilde{x}_{k}\right) \operatorname{Re} e^{2 i \tau \operatorname{Im} \Phi\left(\widetilde{x_{k}}\right)}}{\left|\left(\operatorname{det} \operatorname{Im} \Phi^{\prime \prime}\right)\left(\widetilde{x_{k}}\right)\right|^{\frac{1}{2}}}=0, \quad \tau>0 .
$$

We can choose $\Phi$ such that

$$
\operatorname{Im} \Phi\left(\widetilde{x_{k}}\right) \neq \operatorname{Im} \Phi\left(\widetilde{x_{j}}\right), j \neq k
$$

Let $a\left(\widetilde{x_{k}}\right) \neq 0$. Then Proposition 1.24 implies

$$
q_{1}\left(\widetilde{x_{k}}\right)=q_{2}\left(\widetilde{x_{k}}\right) .
$$

We then show that the non-degenerate critical points of $\Phi$ can be chosen to be a dense set concluding the sketch of the proof of the theorem. We refer to [30] for an analysis of a discrete version of the partial data problem in $2 \mathrm{D}$. The case of Riemannian surfaces was considered in [70]. A improvement on the regularity of the potential is given in [86]. For a study of partial data for a general class of second order elliptic operators see [89]. The case of measurements in disjoint sets is considered in [90].

\subsection{Anisotropic conductivities}

Anisotropic conductivities depend on direction. Muscle tissue in the human body is an important example of an anisotropic conductor. For instance cardiac muscle has a conductivity of 2.3 mho in the transverse direction and 6.3 in the longitudinal direction. The conductivity in this case is represented by a positive definite, smooth, symmetric matrix $\gamma=\left(\gamma^{i j}(x)\right)$ on $\Omega$. Under the assumption of no sources or sinks of current in $\Omega$, the potential $u$ in $\Omega$, given a voltage potential $f$ on $\partial \Omega$, solves the Dirichlet problem

$$
\left\{\begin{aligned}
\sum_{i, j=1}^{n} \frac{\partial}{\partial x_{i}}\left(\gamma^{i j} \frac{\partial u}{\partial x_{j}}\right) & =0 \text { on } \Omega \\
\left.u\right|_{\partial \Omega} & =f .
\end{aligned}\right.
$$


The DN map is defined by

$$
\Lambda_{\gamma}(f)=\left.\sum_{i, j=1}^{n} v^{i} \gamma^{i j} \frac{\partial u}{\partial x_{j}}\right|_{\partial \Omega}
$$

where $v=\left(v^{1}, \ldots, v^{n}\right)$ denotes the unit outer normal to $\partial \Omega$ and $u$ is the solution of (79). The inverse problem is whether one can determine $\gamma$ by knowing $\Lambda_{\gamma}$. Unfortunately, $\Lambda_{\gamma}$ doesn't determine $\gamma$ uniquely. This observation is due to L. Tartar (see [113] for an account). Let $\psi: \bar{\Omega} \rightarrow \bar{\Omega}$ be a $C^{\infty}$ diffeomorphism with $\left.\psi\right|_{\partial \Omega}=I d$ where Id denotes the identity map. We have

$$
\Lambda_{\tilde{\gamma}}=\Lambda_{\gamma}
$$

where

$$
\widetilde{\gamma}=\left(\frac{(D \psi)^{T} \circ \gamma \circ(D \psi)}{|\operatorname{det} D \psi|}\right) \circ \psi^{-1} .
$$

Here $D \psi$ denotes the (matrix) differential of $\psi,(D \psi)^{T}$ its transpose and the composition in (82) is to be interpreted as multiplication of matrices. We have then a large number of conductivities with the same DN map: any change of variables of $\Omega$ that leaves the boundary fixed gives rise to a new conductivity with the same electrostatic boundary measurements. The question is then whether this is the only obstruction to unique identifiability of the conductivity. In two dimensions this has been shown for $L^{\infty}(\Omega)$ conductivities in [12]. This is done by reducing the anisotropic problem to the isotropic one by using isothermal coordinates [2,189] and using Astala and Päivärinta's result in the isotropic case [11]. Earlier results were for $C^{3}$ conductivities using the result of Nachman [134] and for Lipschitz conductivities in [186] using the techniques of [33]. An extension of some of these results to quasilinear anisotropic conductivities can be found in [188]. In three dimensions, as was pointed out in [121], this is a problem of geometrical nature and makes sense for general compact Riemannian manifolds with boundary. Let $(M, g)$ be a compact Riemannian manifold with boundary. The Laplace-Beltrami operator associated to the metric $g$ is given in local coordinates by

$$
\Delta_{g} u=\frac{1}{\sqrt{\operatorname{det} g}} \sum_{i, j=1}^{n} \frac{\partial}{\partial x_{i}}\left(\sqrt{\operatorname{det} g} g^{i j} \frac{\partial u}{\partial x_{j}}\right)
$$

where $\left(g^{i j}\right)$ is the matrix inverse of the matrix $\left(g_{i j}\right)$. Let us consider the Dirichlet problem associated to (83)

$$
\Delta_{g} u=0 \text { on } \Omega,\left.\quad u\right|_{\partial \Omega}=f
$$


We define the DN map in this case by

$$
\Lambda_{g}(f)=\left.\sum_{i, j=1}^{n} v^{i} g^{i j} \frac{\partial u}{\partial x_{j}} \sqrt{\operatorname{det} g}\right|_{\partial \Omega}
$$

The inverse problem is to recover $g$ from $\Lambda_{g}$. We have

$$
\Lambda_{\psi^{*} g}=\Lambda_{g}
$$

where $\psi$ is any $C^{\infty}$ diffeomorphism of $\bar{M}$ which is the identity on the boundary. As usual $\psi^{*} g$ denotes the pull back of the metric $g$ by the diffeomorphism $\psi$. In the case that $M$ is an open, bounded subset of $\mathbb{R}^{n}$ with smooth boundary, it is easy to see ([121]) that for $n \geq 3$

$$
\Lambda_{g}=\Lambda_{\gamma}
$$

where

$$
\left(g_{i j}\right)=\left(\operatorname{det} \gamma^{k l}\right)^{\frac{1}{n-2}}\left(\gamma^{i j}\right)^{-1}, \quad\left(\gamma^{i j}\right)=\left(\operatorname{det} g_{k l}\right)^{\frac{1}{2}}\left(g_{i j}\right)^{-1}
$$

In the two dimensional case there is an additional obstruction since the LaplaceBeltrami operator is conformally invariant. More precisely we have

$$
\Delta_{\alpha g}=\frac{1}{\alpha} \Delta_{g}
$$

for any function $\alpha, \alpha \neq 0$. Therefore we have, for $n=2$,

$$
\Lambda_{\alpha\left(\psi^{*} g\right)}=\Lambda_{g}
$$

for any smooth function $\alpha \neq 0$ so that $\left.\alpha\right|_{\partial M}=1$. Lassas and Uhlmann ([124]) proved that (86) is the only obstruction to unique identifiability of the conductivity for realanalytic manifolds in dimension $n \geq 3$. In the two dimensional case they showed that (89) is the only obstruction to unique identifiability for smooth Riemannian surfaces. Moreover these results assume that the DN map is measured only on an open subset of the boundary. For another proof see [23]. We state the two basic results. Let $\Gamma$ be an open subset $\partial M$. We define for $f$, supp $f \subseteq \Gamma$

$$
\Lambda_{g, \Gamma}(f)=\left.\Lambda_{g}(f)\right|_{\Gamma}
$$

Theorem $1.24(n=2)$ Let $(M, g)$ be a compact Riemannian surface with boundary. Let $\Gamma \subseteq \partial M$ be an open subset. Then $\Lambda_{g}, \Gamma$ determines uniquely the conformal class of $(M, g)$.

Theorem $1.25(n \geq 3)$ Let $(M, g)$ be a real-analytic compact, connected Riemannian manifold with boundary. Let $\Gamma \subseteq \partial M$ be real-analytic and assume that $g$ is realanalytic up to $\Gamma$. Then $\Lambda_{g, \Gamma}$ determines uniquely $(M, g)$ up to an isometry. 
Einstein manifolds are real-analytic in the interior and it was conjectured by Lassas and Uhlmann that they were uniquely determined up to isometry by the DN map. This was proven in [68]. Notice that these results don't assume any condition on the topology of the manifold except for connectedness. An earlier result of [121] assumed that $(M, g)$ was strongly convex and simply connected and $\Gamma=\partial M$ in both results. Theorem 1.25 was extended in [125] to non-compact, connected real-analytic manifolds with boundary. These results were extended to differential forms in [116].

\subsubsection{The Calderón problem on manifolds}

The invariant form on a Riemannian manifold with boundary $(M, g)$ for an isotropic conductivity $\beta$ is given by

$$
\operatorname{div}_{g}\left(\beta \nabla_{g}\right) u=0
$$

where $\operatorname{div}_{g}$ (resp. $\nabla_{g}$ ) denotes divergence (resp. gradient) with respect to the Riemannian metric $g$. This includes the case considered by Calderón with $g$ the Euclidean metric, the anisotropic case by taking $g^{i j}=\gamma^{i j} \beta$ and $\beta=\sqrt{\operatorname{det} g}$. It was shown in [186] for bounded domains of Euclidean space in two dimensions that the isometric class of $(\beta, g)$ is determined uniquely by the DN map associated to (90). In two dimensions, when the metric $g$ is known, it is proven in [77] that one can uniquely determine the conductivity $\beta$. Guillarmou and Tzou [69] have shown that a potential is uniquely determined for the Schrödinger equation $\Delta_{g}-q$, with $\Delta_{g}$ the Laplace-Beltrami operator associated to the metric $g$, generalizing the result of [77]. In dimension $n \geq 3$ it is an open problem whether one can determine the isotropic conductivity $\beta$ from the corresponding DN map associated to (90). As before one can consider the more general problem of recovering the potential $q$ from the DN map associated to $\Delta_{g}-q$. We review below the progress that has been made on this problem based on [52].

\subsubsection{Complex geometrical optics on manifolds}

We review the recent construction of complex geometrical optics construction for a class of Riemannian manifolds based on [52]. In this paper those Riemannian manifolds which admit limiting Carleman weights, were characterized. All such weights in Euclidean space were listed in Theorem 1.13.

Theorem 1.26 If $(M, g)$ is an open manifold having a limiting Carleman weight, then some conformal multiple of the metric $g$ admits a parallel unit vector field. For simply connected manifolds, the converse is also true.

Locally, a manifold admits a parallel unit vector field if and only if it is isometric to the product of an Euclidean interval and another Riemannian manifold. This is an instance of the de Rham decomposition [158]. Thus, if $(M, g)$ has an LCW $\varphi$, one can choose local coordinates in such a way that $\phi(x)=x_{1}$ and

$$
g\left(x_{1}, x^{\prime}\right)=c(x)\left(\begin{array}{cc}
1 & 0 \\
0 & g_{0}\left(x^{\prime}\right)
\end{array}\right),
$$


where $c$ is a positive conformal factor. Conversely, any metric of this form admits $\varphi(x)=x_{1}$ as a limiting weight. In the case $n=2$, limiting Carleman weights in $(M, g)$ are exactly the harmonic functions with non-vanishing differential. Let us now introduce the class of manifolds which admit limiting Carleman weights and for which one can prove uniqueness results. For this we need the notion of simple manifolds [167].

Definition 1.27 A manifold $(M, g)$ with boundary is simple if $\partial M$ is strictly convex, and for any point $x \in M$ the exponential map $\exp _{x}$ is a diffeomorphism from some closed neighborhood of 0 in $T_{x} M$ onto $M$.

Definition 1.28 A compact manifold with boundary $(M, g)$, of dimension $n \geq 3$, is admissible if it is conformal to a submanifold with boundary of $\mathbb{R} \times\left(M_{0}, g_{0}\right)$ where $\left(M_{0}, g_{0}\right)$ is a compact simple $(n-1)$-dimensional manifold.

Examples of admissible manifolds include the following:

1. Bounded domains in Euclidean space, in the sphere minus a point, or in hyperbolic space. In the last two cases, the manifold is conformal to a domain in Euclidean space via stereographic projection.

2. More generally, any domain in a locally conformally flat manifold is admissible, provided that the domain is appropriately small. Such manifolds include locally symmetric 3-dimensional spaces, which have parallel curvature tensor so their Cotton tensor vanishes [54].

3. Any bounded domain $M$ in $\mathbb{R}^{n}$, endowed with a metric which in some coordinates has the form

$$
g\left(x_{1}, x^{\prime}\right)=c(x)\left(\begin{array}{cc}
1 & 0 \\
0 & g_{0}\left(x^{\prime}\right)
\end{array}\right),
$$

with $c>0$ and $g_{0}$ simple, is admissible.

4. The class of admissible metrics is stable under $C^{2}$-small perturbations of $g_{0}$.

The first inverse problem involves the Schrödinger operator

$$
\mathcal{L}_{g, q}=\Delta_{g}-q
$$

where $q$ is a smooth complex valued function on $(M, g)$. We make the standard assumption that 0 is not a Dirichlet eigenvalue of $\mathcal{L}_{g, q}$ in $M$. Then the Dirichlet problem

$$
\left\{\begin{array}{c}
\mathcal{L}_{g, q} u=0 \text { in } M \\
u=f \text { on } \partial M
\end{array}\right.
$$

has a unique solution for any $f \in H^{1 / 2}(\partial M)$, and we may define the DN map

$$
\Lambda_{g, q}:\left.f \mapsto \partial_{\nu} u\right|_{\partial M}
$$

Given a fixed admissible metric, one can determine the potential $q$ from boundary measurements. 
Theorem 1.29 Let $(M, g)$ be admissible, and let $q_{1}$ and $q_{2}$ be two smooth functions on $M$. If $\Lambda_{g, q_{1}}=\Lambda_{g, q_{2}}$, then $q_{1}=q_{2}$.

This result was known previously in dimensions $n \geq 3$ for the Euclidean metric [190] and for the hyperbolic metric [100]. It has been generalized to Maxwell's equations in [106].

\section{Travel time tomography and boundary rigidity}

\subsection{Introduction}

The question of determining the sound speed or index of refraction of a medium by measuring the first arrival times of waves arose in geophysics in an attempt to determine the substructure of the Earth by measuring at the surface of the Earth the travel times of seismic waves. An early success of this inverse method was the estimate by Herglotz [78] and Wiechert and Zoeppritz [207] of the diameter of the Earth and the location of the mantle, crust and core. The assumption used in those papers is that the index of refraction (inverse proportional to the speed) depends only on the radius. A more realistic model is to assume that it depends on position. The inverse kinematic problem can be formulated mathematically as determining a Riemannian metric on a bounded domain (the Earth) given by $d s^{2}=\frac{1}{c^{2}(x)} d x^{2}$, where $c$ is a positive function, from the length of geodesics (travel times) joining points in the boundary. More recently it has been realized, by measuring the travel times of seismic waves, that the inner core of the Earth might exhibit anisotropic behavior, that is the speed of waves depends also on direction there with the fast direction parallel to the Earth's spin axis [44]. Given the complications presented by modeling the Earth as an anisotropic elastic medium we consider a simpler model of anisotropy, namely that the wave speed is given by a symmetric, positive definite matrix $g=\left(g_{i j}\right)(x)$, that is, a Riemannian metric in mathematical terms. The problem is to determine the metric from the lengths of geodesics joining points in the boundary (the surface of the Earth in the motivating example). It is useful to consider a more general and geometric formulation of the problem. Let $(M, g)$ be a compact Riemannian manifold with boundary $\partial M$. Let $d_{g}(x, y)$ denote the geodesic distance between $x$ and $y$, two points in the boundary. This is defined as the infimum of the length of all sufficiently smooth curves joining the two points. The function $d_{g}$ measures the first arrival time of waves joining points of the boundary. One of the inverse problems we discuss in this section is whether we can determine the Riemannian metric $g$ knowing $d_{g}(x, y)$ for any $x \in \partial M, y \in \partial M$. This problem also arose in rigidity questions in Riemannian geometry $[45,67,129]$. The metric $g$ cannot be determined from this information alone. We have $d_{\psi * g}=d_{g}$ for any diffeomorphism $\psi: M \rightarrow M$ that leaves the boundary pointwise fixed, i.e., $\left.\psi\right|_{\partial M}=I d$, where $I d$ denotes the identity map and $\psi^{*} g$ is the pull-back of the metric $g$. The natural question is whether this is the only obstruction to unique identifiability of the metric. It is easy to see that this is not the case. Namely one can construct a metric $g$ and find a point $x_{0}$ in $M$ so that $d_{g}\left(x_{0}, \partial M\right)>\sup _{x, y \in \partial M} d_{g}(x, y)$. For such a metric, $d_{g}$ is independent of a change of $g$ in a neighborhood of $x_{0}$. The hemisphere of the round sphere is another example. Therefore it is necessary to impose some a-priori restrictions 
on the metric. One such restriction is to assume that the Riemannian manifold $(M, g)$ is simple, i.e., $M$ is simply-connected, any geodesic has no conjugate points and $\partial M$ is strictly convex. $\partial M$ is strictly convex if the second fundamental form of the boundary is positive definite in every boundary point. R. Michel conjectured in [129] that simple manifolds are boundary distance rigid, that is $d_{g}$ determines $g$ uniquely up to an isometry which is the identity on the boundary. This is known for simple subspaces of Euclidean space (see [67]), simple subspaces of an open hemisphere in two dimensions (see [130]), simple subspaces of symmetric spaces of constant negative curvature [26], simple two dimensional spaces of negative curvature (see $[46,149]$ ). If one metric is close to the Euclidean metric boundary rigidity was proven in [123] that was improved in [36]. We remark that simplicity of a compact manifold with boundary can be determined from the boundary distance function. Michel's conjecture was proven in generality in [155] in two dimensions and we describe the details of the proof in Sect. 2.2. In the case that both $g_{1}$ and $g_{2}$ are conformal to the Euclidean metric $e$ (i.e., $\left(g_{k}\right)_{i j}=\alpha_{k} \delta_{i j}, k=1,2$ with $\delta_{i j}$ the Kronecker symbol), as mentioned earlier, the problem we are considering here is known in seismology as the inverse kinematic problem. In this case, it has been proven by Mukhometov in two dimensions [131] that if $\left(M, g_{i}\right), i=1,2$ is simple and $d_{g_{1}}=d_{g_{2}}$, then $g_{1}=g_{2}$. More generally the same method of proof shows that if $\left(M, g_{i}\right), i=1,2$, are simple compact Riemannian manifolds with boundary and they are in the same conformal class then the metrics are determined by the boundary distance function. More precisely we have:

Theorem 2.1 Let $\left(M, g_{i}\right), i=1,2$ be simple compact Riemannian manifolds with boundary of dimension $n \geq 2$. Assume $g_{1}=\rho g_{2}$ for a positive, smooth function $\rho,\left.\rho\right|_{\partial M}=1$ and $d_{g_{1}}=d_{g_{2}}$ then $g_{1}=g_{2}$.

This result and a stability estimate were proven in [131]. We remark that in this case the diffeomorphism $\psi$ that is present in the general case must be the identity if the metrics are conformal to each other. For related results and generalizations see $[25,27,45,60,133]$.

In Sect. 2.2 we consider the boundary rigidity in the two dimensional case and in Sect. 2.3 in the higher dimensional case. In Sect. 2.4 we discuss some results on the non-simple case where the measurements are given by the scattering relation. Roughly speaking one measures the point of exit and direction of exit of a geodesic for which we know the point of entrance and direction of entrance besides this we also know the travel time, that is the length of that geodesic.

\subsection{Boundary rigidity in two dimensions}

We will sketch in this section the proof of the following result.

Theorem 2.2 Let $\left(M, g_{i}\right), i=1,2$ be two dimensional simple compact Riemannian manifolds with boundary. Assume

$$
d_{g_{1}}(x, y)=d_{g_{2}}(x, y) \quad \forall(x, y) \in \partial M \times \partial M .
$$


Then there exists a diffeomorphism $\psi: M \rightarrow M,\left.\psi\right|_{\partial M}=I d$, so that

$$
g_{2}=\psi^{*} g_{1}
$$

The proof of Theorem 2.2 involves a connection between the scattering relation and the Dirichlet-to-Neumann map (DN) associated to the Laplace-Beltrami operator discussed in section. In Sect. 2.2.1 we define the scattering relation which quantizes the scattering operator. In Sect. 2.2.2 we discuss the geodesic X-ray transform and compute the commutator of the fiberwise Hilbert transform and geodesic flow (see Theorem 2.7 and Theorem 2.8). In Sect. 2.2.3 we discuss the main step of the proof of Theorem 2.2 which consists in showing that, under the assumptions of the theorem, we can determine the Dirichlet-to-Neumann map if we know the scattering relation. We also sketch the proof of Theorem 2.9. In Sect. 2.2.4 we use the connection indicated in Sect. 2.2.1, to give a characterization of the range of the geodesic X-ray transform in terms of the scattering relation and we give Fredholm type inversion formulas for the geodesic X-ray transform acting on scalar functions and vector fields.

\subsubsection{The scattering relation}

Suppose we have a Riemannian metric in Euclidean space which is the Euclidean metric outside a compact set. The inverse scattering problem for metrics is to determine the Riemannian metric by measuring the scattering operator (see [72]). A similar obstruction to the boundary rigidity problem occurs in this case with the diffeomorphism $\psi$ equal to the identity outside a compact set. It was proven in [72] that from the wave front set of the scattering operator, one can determine, under some non-trapping assumptions on the metric, the scattering relation on the boundary of a large ball. This uses high frequency information of the scattering operator. In the semiclassical setting Alexandrova has shown for a large class of operators that the scattering operator associated to potential and metric perturbations of the Euclidean Laplacian is a semiclassical Fourier integral operator quantized by the scattering relation [8]. The scattering relation maps the point and direction of a geodesic entering the manifold to the point and direction of exit of the geodesic. We proceed to define in more detail the scattering relation and its relation with the boundary distance function. Let $v$ denote the unit-inner normal to $\partial M$. We denote by $S(M) \rightarrow M$ the unit-sphere bundle over $M$ :

$$
S(M)=\bigcup_{x \in M} S_{x}, \quad S_{x}=\left\{\xi \in T_{x}(M):|\xi|_{g}=1\right\}
$$

$S(M)$ is a $(2 \operatorname{dim} M-1)$-dimensional compact manifold with boundary, which can be written as the union $\partial \Omega(M)=\partial_{+} S(M) \cup \partial_{-} S(M)$

$$
\partial_{ \pm} S(M)=\{(x, \xi) \in \partial S(M), \pm(v(x), \xi) \geq 0\} .
$$

The manifold of inner vectors $\partial_{+} S(M)$ and outer vectors $\partial_{-} S(M)$ intersect at the set of tangent vectors 


$$
\partial_{0} S(M)=\{(x, \xi) \in \partial S(M), \quad(v(x), \xi)=0\} .
$$

Let $(M, g)$ be an n-dimensional compact manifold with boundary. We say that $(M, g)$ is non-trapping if each maximal geodesic is finite. Let $(M, g)$ be non-trapping and the boundary $\partial M$ is strictly convex. Denote by $\tau(x, \xi)$ the length of the geodesic $\gamma(x, \xi, t), t \geq 0$, starting at the point $x$ in the direction $\xi \in S_{x}$. This function is smooth on $S(M) \backslash \partial_{0} S(M)$. The function $\tau^{0}=\left.\tau\right|_{\partial S(M)}$ is equal to zero on $\partial_{-} S(M)$ and is smooth on $\partial_{+} S(M)$. Its odd part with respect to $\xi$

$$
\tau_{-}^{0}(x, \xi)=\frac{1}{2}\left(\tau^{0}(x, \xi)-\tau^{0}(x,-\xi)\right)
$$

is a smooth function.

Definition 2.3 Let $(M, g)$ be non-trapping with strictly convex boundary. The scattering relation $\alpha: \partial S(M) \rightarrow \partial S(M)$ is defined by

$$
\alpha(x, \xi)=\left(\gamma\left(x, \xi, 2 \tau_{-}^{0}(x, \xi)\right), \dot{\gamma}\left(x, \xi, 2 \tau_{-}^{0}(x, \xi)\right)\right) .
$$

The scattering relation is a diffeomorphism $\partial S(M) \rightarrow \partial S(M)$. Notice that $\left.\alpha\right|_{\partial_{+} S(M)}: \partial_{+} S(M) \rightarrow \partial_{-} S(M),\left.\alpha\right|_{\partial_{-} S(M)}: \partial_{-} S(M) \rightarrow \partial_{+} S(M)$ are diffeomorphisms as well. Obviously, $\alpha$ is an involution, $\alpha^{2}=i d$ and $\partial_{0} S(M)$ is the hypersurface of its fixed points, $\alpha(x, \xi)=(x, \xi),(x, \xi) \in \partial_{0} S(M)$. A natural inverse problem is whether the scattering relation determines the metric $g$ up to an isometry which is the identity on the boundary. This information takes into account all the travel times not just the first arrivals. In the case that $(M, g)$ is a simple manifold, and we know the metric at the boundary (and this is determined if $d_{g}$ is known, see [129], knowing the scattering relation is equivalent to knowing the boundary distance function ([129]) so that we concentrate on studying the scattering relation. We introduce the operators of even and odd continuation with respect to $\alpha$ :

$$
\begin{aligned}
& A_{ \pm} w(x, \xi)=w(x, \xi), \quad(x, \xi) \in \partial_{+} S(M) \\
& A_{ \pm} w(x, \xi)= \pm\left(\alpha^{*} w\right)(x, \xi), \quad(x, \xi) \in \partial_{-} S(M)
\end{aligned}
$$

The scattering relation preserves the measure $|(\xi, v)| d \Sigma,(d \Sigma$ is the measure of the boundary $\partial S(M)$ induced by the metric $g$ ) and therefore the operators $A_{ \pm}$: $L_{\mu}^{2}\left(\partial_{+} S(M)\right) \rightarrow L_{|\mu|}^{2}(\partial S(M))$ are bounded, where $L_{|\mu|}^{2}(\partial S(M))$ is the real Hilbert space with scalar product

$$
(u, v)_{L_{|\mu|}^{2}(\partial S(M))}=\int_{\partial S(M)}|\mu| u v d \Sigma, \quad \mu=(\xi, v) .
$$

and $L_{\mu}^{2}\left(\partial_{+} S(M)\right)$ is the real Hilbert space with scalar product

$$
(u, v)_{L_{\mu}^{2}\left(\partial_{+} S(M)\right)}=\int_{\partial_{+} S(M)} \mu u v d \Sigma .
$$


The adjoint of $A_{ \pm}$is a bounded operator $A_{ \pm}^{*}: L_{|\mu|}^{2}(\partial S(M)) \rightarrow L_{\mu}^{2}\left(\partial_{+} S(M)\right)$ given by

$$
A_{ \pm}^{*} u=\left.(u \pm u \circ \alpha)\right|_{\partial_{+} S(M)}
$$

\subsubsection{The geodesic X-ray transform}

The X-ray transform integrates a function along lines. Radon found in 1917 an inversion formula in two dimensions to determine a function knowing the X-ray transform. This formula is non-local in the sense that in order to find the function at a point $x$ one needs to know the integral of the function along lines far from the point. Radon's inversion formula has been implemented numerically using the filtered backprojection algorithm which is used today in CT scans. Another important transform in medical imaging and other applications is the Doppler transform which integrates a vector field along lines. The motivation is ultrasound Doppler tomography. It is known that blood flow is irregular and faster around tumor tissue than in normal tissue and Doppler tomography attempts to reconstruct the blood flow pattern. Mathematically the problem is to what extend a vector field is determined from its integral along lines. In this paper we consider the case of integrating functions and vector fields along geodesics of a Riemannian metric. This arises in geophysics since the ray paths are no longer straight lines. We obtain inversion formulas for the constant curvature case and Fredholm type formulas in general which are non-local. We define next the geodesic X-ray transform for any compact Riemannian manifold $(M, g)$ with boundary of any dimension. We embed $(M, g)$ into a compact Riemannian manifold $(\widetilde{M}, g)$ with no boundary. Let $\varphi_{t}$ be the geodesic flow on $S(\widetilde{M})$ and $\mathcal{H}=\left.\frac{d}{d t} \varphi_{t}\right|_{t=0}$ be the geodesic vector field. Let $u^{f}$ be the solution of the boundary value problem

$$
\mathcal{H} u=-f,\left.\quad u\right|_{\partial_{-} S(M)}=0,
$$

which can be written as

$$
u^{f}(x, \xi)=\int_{0}^{\tau(x, \xi)} f\left(\varphi_{t}(x, \xi)\right) d t, \quad(x, \xi) \in S(M) .
$$

In particular

$$
\mathcal{H} \tau=-1
$$

The trace

$$
I f=\left.u^{f}\right|_{\partial_{+} S(M)}
$$

is called the geodesic X-ray transform of the function $f$. If the manifold $(M, g)$ is non-trapping and has a strictly convex boundary the operator $I: C^{\infty}(S(M)) \rightarrow$ $C^{\infty}\left(\partial_{+} S(M)\right)$. Clearly a function $f$ is not determined by its geodesic X-ray transform 
alone, since it depends on more variables than If . We consider the geodesic X-ray transform acting on symmetric tensor fields. We denote by $f_{m}(x, \xi)$ an homogeneous polynomial of degree $m$ with respect to $\xi$, induced by the symmetric tensor field $f$ on $(M, g)$ of $m$ degree:

$$
f_{m}(x, \xi)=f_{i_{1} \ldots i_{m}}(x) \xi^{i_{1}} \ldots \xi^{i_{m}}
$$

The operator $I_{m}$, defined by

$$
I_{m} f=I f_{m}
$$

is called the geodesic X-ray transform of the symmetric tensor field. If the manifold $(M, g)$ is non-trapping and the boundary $\partial M$ is strictly convex $I_{m}: C^{\infty}\left(M, S_{m}(M)\right) \rightarrow$ $C^{\infty}\left(\partial_{+} S(M)\right)$, where $S_{m}(M)$ denotes the bundle of symmetric tensors over $(M, g)$. It is known that any symmetric smooth enough tensor field $f$ may be decomposed in a potential and solenoidal part [167]:

$$
f=d p+h,\left.\quad p\right|_{\partial M}=0, \quad \delta h=0,
$$

where $\delta$ denotes the divergence and $d=\sigma \nabla$ is the symmetric part of covariant derivative. It is easy to see that the geodesic X-ray transform of the potential part $d p$ is zero. We denote by $C_{s o l}^{\infty}\left(M, S_{m}(M)\right)$ the space of smooth solenoidal symmetric tensor fields. The case of the geodesic X-ray transform acting on functions independent of $\xi$ and the geodesic X-ray transform acting on vector fields which, following the notation above, are denoted by $I_{0}$ and $I_{1}$ respectively. It is known that $I_{0}$ is injective on simple manifolds $[131,132,161]$ and that $I_{1}$ is injective acting on solenoidal vector fields on simple manifolds [10] and for tensors of order two in [168]. Recently in [151] it has been proven injectivity of $I_{m}$ for all $m$ for simple two-dimensional manifolds. It was shown in [170] that $I_{2}$ is injective for surfaces with no focal points. We mention also that the transform $I_{2}$ arises in the linearization of the boundary rigidity problem (see [167]). We define $\psi: S(M) \rightarrow \partial_{+} S(M)$ by

$$
\psi(x, \xi)=\varphi_{-\tau(x,-\xi)}(x, \xi),(x, \xi) \in S(M) .
$$

So, $\varphi$ is a retract which maps vector $(x, \xi)$ along the geodesic $\gamma(x, \xi, t)$ in the back direction into an incoming vector. The solution of the boundary value problem for the transport equation

$$
\mathcal{H} u=0,\left.\quad u\right|_{\partial_{+} S(M)}=w
$$

can be written in the form

$$
u=w_{\psi}=w \circ \psi
$$


The adjoint of the operator $I_{m}$ is the bounded operator $I_{m}^{*}: L_{\mu}^{2}\left(\partial_{+} S(M)\right) \rightarrow$ $L^{2}\left(M, S_{m}(M)\right)$ which is given by

$$
\left(I_{m}^{*} w\right)^{i_{1} \ldots i_{m}}(x)=\int_{S_{x}} w_{\psi}(x, \xi) \xi^{i_{1}} \ldots \xi^{i_{m}} d S_{x} .
$$

The Hilbert space $L^{2}\left(M, S_{m}(M)\right)$ may be considered as subspace of $L^{2}(S(M))$ of homogeneous polynomials with respect to $\xi$ of degree $m$. The field $I_{m}^{*} w$ is solenoidal in the sense of the theory of distributions. Notice, that the adjoint of the bounded operator $I: L^{2}(S(M)) \rightarrow L_{\mu}^{2}\left(\partial_{+} S(M)\right)$ is given by

$$
I^{*} w=w_{\psi}
$$

We also remark that by the fundamental theorem of calculus we have

$$
I \mathcal{H} f=\left.(f \circ \alpha-f)\right|_{\partial_{+} S(M)}=-A_{-}^{*} f^{0}, f^{0}=\left.f\right|_{\partial S(M)} .
$$

The space $C_{\alpha}^{\infty}\left(\partial_{+} S(M)\right)$ is defined by

$$
C_{\alpha}^{\infty}\left(\partial_{+} S(M)\right)=\left\{w \in C^{\infty}\left(\partial_{+} S(M)\right): w_{\psi} \in C^{\infty}(S(M))\right\}
$$

In [155] the following characterization of the space of smooth solutions of the transport equation was given

\section{Lemma 2.4}

$$
C_{\alpha}^{\infty}\left(\partial_{+} S(M)\right)=\left\{w \in C^{\infty}\left(\partial_{+} S(M)\right): A_{+} w \in C^{\infty}(\partial S(M))\right\}
$$

In the scalar case the following result holds on the solvability of $I_{0}^{*}[155,156]$.

Theorem 2.5 Let $(M, g)$ be a simple, compact Riemannian manifold with boundary. Then the operator $I_{0}^{*}: C_{\alpha}^{\infty}\left(\partial_{+} S(M)\right) \rightarrow C^{\infty}(M)$ is onto.

The analog result for vector fields was proven in [50].

Theorem 2.6 Let $(M, g)$ be a simple, compact Riemannian manifold with boundary. Then for any field $v \in C_{\text {sol }}^{\infty}(M, T(M))$ there exists a function $w \in C_{\alpha}^{\infty}\left(\partial_{+} S(M)\right)$ such that

$$
v=I_{1}^{*} w .
$$

Now we define the Hilbert transform in the $\xi$ variable:

$$
H u(x, \xi)=\frac{1}{2 \pi} \int_{S_{x}} \frac{1+(\xi, \eta)}{\left(\xi_{\perp}, \eta\right)} u(x, \eta) d S_{x}(\eta), \quad \xi \in S_{x},
$$


where the integral is understood as a principle value integral. Here $\perp$ means a $90^{\circ}$ degree rotation. In coordinates $\left(\xi_{\perp}\right)_{i}=\varepsilon_{i j} \xi^{j}$, where

$$
\varepsilon=\sqrt{\operatorname{det} g}\left(\begin{array}{cc}
0 & 1 \\
-1 & 0
\end{array}\right) .
$$

The Hilbert transform $H$ transforms even (respectively odd) functions with respect to $\xi$ to even (respectively odd) ones. If $H_{+}$(respectively $H_{-}$) is the even (respectively odd) part of the operator $H$ :

$$
\begin{aligned}
& H_{+} u(x, \xi)=\frac{1}{2 \pi} \int_{S_{x}} \frac{(\xi, \eta)}{\left(\xi_{\perp}, \eta\right)} u(x, \eta) d S_{x}(\eta), \\
& H_{-} u(x, \xi)=\frac{1}{2 \pi} \int_{S_{x}} \frac{1}{\left(\xi_{\perp}, \eta\right)} u(x, \eta) d S_{x}(\eta)
\end{aligned}
$$

and $u_{+}, u_{-}$are the even and odd parts of the function $u$, then $H_{+} u=H u_{+}, H_{-} u=$ $\mathrm{Hu} u_{-}$.

We introduce the notation $\mathcal{H}_{\perp}=\left(\xi_{\perp}, \nabla\right)=-\left(\xi, \nabla_{\perp}\right)$, where $\nabla_{\perp}=\varepsilon \nabla$ and $\nabla$ is the covariant derivative with respect to the metric $g$. The following commutator formula for the geodesic vector field and the Hilbert transform, is a crucial ingredient in the proofs of the main theorems surveyed in this paper (see [155]).

Theorem 2.7 Let $(M, g)$ be a two dimensional Riemannian manifold. For any smooth function u on $S(M)$ we have the identity

$$
[H, \mathcal{H}] u=\mathcal{H}_{\perp} u_{0}+\left(\mathcal{H}_{\perp} u\right)_{0}
$$

where

$$
u_{0}(x)=\frac{1}{2 \pi} \int_{S_{x}} u(x, \xi) d S_{x}(\xi)
$$

is the average value.

We define

$$
P_{-}=A_{-}^{*} H_{-} A_{+}, \quad P_{+}=A_{-}^{*} H_{+} A_{+} .
$$

If the manifold $(M, g)$ is simple, the following factorizations hold:

\section{Theorem 2.8}

$$
P_{-}=-\frac{1}{2 \pi} I \delta_{\perp} I_{1}^{*}, \quad P_{+}=-\frac{1}{2 \pi} I_{1} \nabla_{\perp} I_{0}^{*}
$$




\subsubsection{The scattering relation and the Dirichlet-to-Neumann Map}

The DN map for the Laplace-Beltrami operator was defined in Sect. 1.7. The connection in two dimensions between the DN map and the scattering relation is given by

Theorem 2.9 Let $\left(M, g_{i}\right), i=1,2$, be compact, simple two dimensional Riemannian manifolds with boundary. Assume that $\alpha_{g_{1}}=\alpha_{g_{2}}$. Then $\Lambda_{g_{1}}=\Lambda_{g_{2}}$.

The proof of Theorem 2.2 is reduced then to the proof of Theorem 2.9. In fact from Theorem 2.9 and Theorem 1.24 we obtain that we can determine the conformal class of the metric up to an isometry which is the identity on the boundary. Now by Theorem 2.1 we have that the conformal factor must be one proving that the metrics are isometric via a diffeomorphism which is the identity at the boundary. In other words $d_{g_{1}}=d_{g_{2}}$ implies that $\alpha_{g_{1}}=\alpha_{g_{2}}$. By Theorem $2.9 \Lambda_{g_{1}}=\Lambda_{g_{2}}$. By Theorem 1.24 , there exists a diffeomorphism $\psi: M \longrightarrow M,\left.\psi\right|_{\partial M}=$ Identity and a function $\beta \neq 0,\left.\beta\right|_{\partial M}=$ identity such that $g_{1}=\beta \psi^{*} g_{2}$. By Mukhometov's theorem $\beta=1$ showing that $g_{1}=\psi^{*} g_{2}$ proving Theorem 2.2. Before starting the proof of Theorem 2.9 we recall that Michel [130] has proven that for two dimensional Riemannian manifolds with strictly convex boundary one can determine from the boundary distance function, up to the natural obstruction, all the derivatives of the metric at the boundary. This result was generalized to any dimensions in [123]. The proof of Theorem 2.9 consists in showing that from the scattering relation we can determine the traces at the boundary of conjugate harmonic functions, which is equivalent information to knowing the DN map associated to the Laplace-Beltrami operator.

Sketch of the proof of Theorem 2.9 Let $\left(h, h_{*}\right)$ be a pair of conjugate harmonic functions on $M$,

$$
\nabla h=\nabla_{\perp} h_{*}, \quad \nabla h_{*}=-\nabla_{\perp} h .
$$

Notice, that $\delta \nabla=\triangle$ is the Laplace-Beltrami operator and $\delta \nabla_{\perp}=0$. Let $I_{0}^{*} w=h$. Since $I_{1} \mathcal{H}_{\perp} h=I_{1} \mathcal{H} h_{*}=-A_{-}^{*} h_{*}^{0}$, where $h_{*}^{0}=\left.h_{*}\right|_{\partial M}$, we obtain from the second identity (93)

$$
2 \pi A_{-}^{*} H_{+} A_{+} w=-A_{-}^{*} h_{*}^{0} .
$$

The following theorem gives the key to obtain the DN map from the scattering relation.

Theorem 2.10 Let $M$ be a 2-dimensional simple manifold. Let $w \in C_{\alpha}^{\infty}\left(\partial_{+} S(M)\right)$ and $h_{*}$ the harmonic continuation of function $h_{*}^{0}$. Then the equation (94) holds iff the functions $h=I_{0}^{*} w$ and $h_{*}$ are conjugate harmonic functions.

In summary we have the following procedure to obtain the DN map from the scattering relation. For a given smooth function $h_{*}^{0}$ on $\partial M$ we find a solution $w \in$ $C_{\alpha}^{\infty}\left(\partial_{+} S(M)\right)$ of the equation (94). Then the functions $h^{0}=2 \pi\left(A_{+} w\right)_{0}$ (notice, that $\left.2 \pi\left(A_{+} w\right)_{0}=\left.I_{0}^{*} w\right|_{\partial M}\right)$ and $h_{*}^{0}$ are the traces of conjugate harmonic functions. It is easy to see that this gives the DN map. 


\subsubsection{Range and inversion of the geodesic X-ray transform}

Let $T(M)$ be the tangent bundle of $M$. We denote by $\delta$ the divergence operator $\delta$ : $\left.C^{\infty}(M, T M)\right) \rightarrow C^{\infty}(M)$. In local coordinates this is given by $\delta u=g^{k j} \nabla_{k} u_{j}$ using Einstein's summation convention. We define the operator $\delta_{\perp}: C^{\infty}(M, T(M)) \rightarrow$ $C^{\infty}(M)$ by

$$
\delta_{\perp} u=-\delta u_{\perp} .
$$

Then

$$
\delta_{\perp} \nabla_{\perp} f=\delta \nabla f=\Delta f, \quad \delta_{\perp} \nabla f=-\delta \nabla_{\perp} f=0 .
$$

We now give the characterization of the range of $I_{0}$ and $I_{1}$ in terms of the scattering relation only. We have that these are the projections of the operators $P_{-}, P_{+}$respectively. For the details see [157].

Theorem 2.11 Let $(M, g)$ be simple two dimensional compact Riemannian manifold with boundary. Then i) The maps

$$
\begin{gathered}
\delta_{\perp} I_{1}^{*}: C_{\alpha}^{\infty}\left(\partial_{+} S(M)\right) \rightarrow C^{\infty}(M), \\
\nabla_{\perp} I_{0}^{*}: C_{\alpha}^{\infty}\left(\partial_{+} S(M)\right) \rightarrow C_{\text {sol }}^{\infty}(M, T(M))
\end{gathered}
$$

are onto. ii). A function $u \in C^{\infty}\left(\partial_{+} S(M)\right)$ belong to Range $I_{0}$ iff $u=P_{-} w, w \in$ $C_{\alpha}^{\infty}\left(\partial_{+} S(M)\right)$. iii). A function $u \in C^{\infty}\left(\partial_{+} S(M)\right)$ belong to Range $I_{1}$ iff $u=$ $P_{+} w, w \in C_{\alpha}^{\infty}\left(\partial_{+} S(M)\right)$.

Proposition 2.12 The operator $W: C_{0}^{\infty}(M) \rightarrow C^{\infty}(M)$, defined by

$$
W f=\left(\mathcal{H}_{\perp} u^{f}\right)_{0}
$$

can be extended to a smoothing operator $W: L^{2}(M) \rightarrow C^{\infty}(M)$.

We remark that in the case of constant Gaussian curvature $W=0$ and this does not depend on whether the metric has conjugate points so that the inversion formulas of Theorem 2.13 hold for all two dimensional manifolds with boundary with constant curvature. The inversion formulas are (see [157])

Theorem 2.13 Let $(M, g)$ be a two-dimensional simple manifold. Then we have

$$
\begin{aligned}
f+W^{2} f & =\frac{1}{2 \pi} \delta_{\perp} I_{1}^{*} w, \quad w=\left.\frac{1}{2} \alpha^{*} H\left(I_{0} f\right)^{-}\right|_{\partial_{+} S(M)}, \quad f \in L^{2}(M), \\
h+\left(W^{*}\right)^{2} h & =\frac{1}{2 \pi} I_{0}^{*} w, \quad w=\left.\frac{1}{2} \alpha^{*} H\left(I_{1} \mathcal{H}_{\perp} h\right)^{+}\right|_{\partial_{+} S(M)}, h \in H_{0}^{1}(M),
\end{aligned}
$$

where $W, W^{*}: L^{2}(M) \rightarrow C^{\infty}(M)$. In the case of a manifold of constant curvature $W=0, \quad W^{*}=0$. Here $W^{*}$ denotes the adjoint of $W$. 


\subsubsection{Remarks}

The Hilbert transform for 2-dimensional Riemannian manifolds is the map that relates the restrictions on the boundary of conjugate harmonic functions. In this sense the Hilbert transform, up to a constant, is just the Dirichlet-to-Neumann (DN) map. In Sect. 2.2 we fixed a point $x$ and started with the microlocal Hilbert transform on the circle $S_{x}$ in the tangent space with Euclidean metric and we ended with the global Hilbert transform (the DN map). The scattering relation and the boundary distance function are determined by the singularities of the DN map associated to the wave equation for the Laplace-Beltrami operator, the so-called hyperbolic (or dynamic) Dirichlet-to-Neumann map [201]. We have found, in two dimensions, a connection between the scattering relation and the elliptic Dirichlet-to-Neumann map which led to a solution of the boundary rigidity problem in two dimensions. Is there a similar connection in higher dimensions?

\subsection{Boundary rigidity and tensor tomography in dimensions $n \geq 3$}

For an earlier review see [177]. In [178], it was proven a local result for metrics in a small neighborhood of the Euclidean one. This result was used in [123] to prove a semiglobal solvability result assuming that one metric is close to the Euclidean and the other has bounded curvature. As it was mentioned earlier it is known [167], that a linearization of the boundary rigidity problem near a simple metric $g$ is given by the following integral geometry problem: recover a symmetric tensor of order 2 , which in any coordinates is given by $f=\left(f_{i j}\right)$, by the geodesic X-ray transform

$$
I_{g} f(\gamma)=\int f_{i j}(\gamma(t)) \dot{\gamma}^{i}(t) \dot{\gamma}^{j}(t) \mathrm{d} t
$$

known for all geodesics $\gamma$ in $M$. In this section we denote by $I_{g}$ the geodesic X-ray transform of tensors of order two. It can be easily seen that $I_{g} d v=0$ for any vector field $v$ with $\left.v\right|_{\partial M}=0$, where $d v$ denotes the symmetric differential

$$
[d v]_{i j}=\frac{1}{2}\left(\nabla_{i} v_{j}+\nabla_{j} v_{i}\right)
$$

and $\nabla_{k} v$ denote the covariant derivatives of the vector field $v$. This is the linear version of the fact that $d_{g}$ does not change on $(\partial M)^{2}:=\partial M \times \partial M$ under an action of a diffeomorphism as above. The natural formulation of the linearized problem is therefore that $I_{g} f=0$ implies $f=d v$ with $v$ vanishing on the boundary. We will refer to this property as s-injectivity of $I_{g}$. More precisely, we have.

Definition 2.14 We say that $I_{g}$ is s-injective in $M$, if $I_{g} f=0$ and $f \in L^{2}(M)$ imply $f=d v$ with some vector field $v \in H_{0}^{1}(M)$.

Any symmetric tensor $f \in L^{2}(M)$ admits an orthogonal decomposition $f=$ $f^{s}+d v$ into a solenoidal and potential parts with $v \in H_{0}^{1}(M)$, and $f^{s}$ divergence 
free, i.e., $\delta f^{s}=0$, where $\delta$ is the adjoint operator to $-d$ given by $[\delta f]_{i}=g^{j k} \nabla_{k} f_{i j}$ [167]. Therefore, $I_{g}$ is s-injective, if it is injective on the space of solenoidal tensors. The inversion of $I_{g}$ is a problem of independent interest in integral geometry, also called tensor tomography. We first survey the recent results on this problem. S-injectivity, respectively injectivity for 1-tensors (1-forms) and functions is known, see [167] for references. S-injectivity of $I_{g}$ was proved in [154] for metrics with negative curvature, in [167] for metrics with small curvature and in [170] for Riemannian surfaces with no focal points. A conditional and non-sharp stability estimate for metrics with small curvature is also established in [167]. In [179], stability estimates for s-injective metrics [see (99) below] were shown and sharp estimates about the recovery of a 1-form $f=f_{j} d x^{j}$ and a function $f$ from the associated $I_{g} f$ which is defined by

$$
I_{g} f(\gamma)=\int f_{i}(\gamma(t)) \dot{\gamma}^{i}(t) d t
$$

The stability estimates proven in [179] were used to prove local uniqueness for the boundary rigidity problem near any simple metric $g$ with s-injective $I_{g}$. Similarly to [195], we say that $f$ is analytic in the set $K$ (not necessarily open), if it is real analytic in some neighborhood of $K$. The results that follow in this section are based on [181]. The first main result we discuss is about s-injectivity for simple analytic metrics.

Theorem 2.15 Let $g$ be a simple, real analytic metric in $M$. Then $I_{g}$ is s-injective.

Sketch of the proof Note that a simple metric $g$ in $M$ can be extended to a simple metric in some $M_{1}$ with $M \Subset M_{1}$. A simple manifold is diffeomorphic to a (strictly convex) domain $\Omega \subset \mathbf{R}^{n}$ with the Euclidean coordinates $x$ in a neighborhood of $\Omega$ and a metric $g(x)$ there. For this reason, it is enough to prove the results of this section for domains $\Omega$ in $\mathbf{R}^{n}$ provided with a Riemannian metric $g$. The proof of Theorem 2.15 is based on the following. For smooth metrics, the normal operator $N_{g}=I_{g}^{*} I_{g}$ is a pseudodifferential operator with a non-trivial null space which is given by

$$
\left(N_{g} f\right)_{k l}(x)=\frac{2}{\sqrt{\operatorname{det} g}} \int \frac{f^{i j}(y)}{d_{g}(x, y)^{n-1}} \frac{\partial d_{g}}{\partial y^{i}} \frac{d_{g}}{\partial y^{j}} \frac{\partial d_{g}}{\partial x^{k}} \frac{\partial d_{g}}{\partial x^{l}} \operatorname{det} \frac{\partial^{2}\left(d_{g}^{2} / 2\right)}{\partial x \partial y} \mathrm{~d} y, \quad x \in \Omega .
$$

In the case that the metric $g$ is real-analytic, $N_{g}$ is an analytic pseudodifferential operator with a non-trivial kernel. We construct an analytic parametrix, using the analytic pseudodifferential calculus in [195], that allows us to reconstruct the solenoidal part of a tensor field from its geodesic X-ray transform, up to a term that is analytic near $\Omega$. If $I_{g} f=0$, we show that for some $v$ vanishing on $\partial \Omega, \widetilde{f}:=f-d v$ must be flat at $\partial \Omega$ and analytic in $\bar{\Omega}$, hence $\widetilde{f}=0$. This is similar to the known argument that an analytic elliptic pseudodifferential operator resolves the analytic singularities, hence cannot have compactly supported functions in its kernel. In our case we have a non-trivial kernel, and complications due to the presence of a boundary, in particular a lost of one derivative. For more details see [181]. 
As shown in [179], the s-injectivity of $I_{g}$ for analytic simple $g$ implies a stability estimate for $I_{g}$. In next theorem we show something more, namely that we have a stability estimate for $g$ in a neighborhood of each analytic metric, which leads to stability estimates for generic metrics. As above, let $M_{1} \ni M$ be a compact manifold which is a neighborhood of $M$ and $g$ extends as a simple metric there. We always assume that our tensors are extended as zero outside $M$, which may create jumps at $\partial M$. Define the normal operator $N_{g}=I_{g}^{*} I_{g}$, where $I_{g}^{*}$ denotes the operator adjoint to $I_{g}$ with respect to an appropriate measure. We showed in [179] that $N_{g}$ is a pseudodifferential operator in $M_{1}$ of order -1 . We introduce the norm $\|\cdot\|_{\widetilde{H}^{2}\left(M_{1}\right)}$ of $N_{g} f$ in $M_{1} \supset M$ in the following way. Choose $\chi \in C_{0}^{\infty}$ equal to 1 near $\partial M$ and supported in a small neighborhood of $\partial M$ and let $\chi=\sum_{j=1}^{J} \chi_{j}$ be a partition of $\chi$ such that for each $j$, on supp $\chi_{j}$ we have coordinates $\left(x_{j}^{\prime}, x_{j}^{n}\right)$, with $x_{j}^{n}$ a normal coordinate. Set

$$
\begin{gathered}
\|f\|_{\widetilde{H}^{1}}^{2}=\int \sum_{j=1}^{J} \chi_{j}\left(\sum_{i=1}^{n-1}\left|\partial_{x_{j}^{i}} f\right|^{2}+\left|x_{j}^{n} \partial_{x_{j}^{n}} f\right|^{2}+|f|^{2}\right) \mathrm{d} x, \\
\left\|N_{g} f\right\|_{\widetilde{H}^{2}\left(M_{1}\right)}=\sum_{i=1}^{n}\left\|\partial_{x^{i}} N_{g} f\right\|_{\widetilde{H}^{1}}+\left\|N_{g} f\right\|_{H^{1}\left(M_{1}\right)} .
\end{gathered}
$$

In other words, in addition to derivatives up to order $1,\left\|N_{g} f\right\|_{\widetilde{H}^{2}\left(M_{1}\right)}$ includes also second derivatives near $\partial M$ but they are realized as first derivatives of $\nabla N_{g} f$ tangent to $\partial M$. The reason to use the $\widetilde{H}^{2}\left(M_{1}\right)$ norm, instead of the stronger $H^{2}\left(M_{1}\right)$ norm, is that this allows us to work with $f \in H^{1}(M)$, not only with $f \in H_{0}^{1}(M)$, since for such $f$, extended as 0 outside $M$, we still have that $N_{g} f \in \widetilde{H}^{2}\left(M_{1}\right)$, see [179]. On the other hand, $f \in H^{1}(M)$ implies $N_{g} f \in \widetilde{H}^{2}\left(M_{1}\right)$ despite the possible jump of $f$ at $\partial M$. Our stability estimate for the linearized inverse problem is as follows:

Theorem 2.16 There exists $k_{0}$ such that for each $k \geq k_{0}$, the set $\mathcal{G}^{k}(M)$ of simple $C^{k}(M)$ metrics in $M$ for which $I_{g}$ is s-injective is open and dense in the $C^{k}(M)$ topology. Moreover, for any $g \in \mathcal{G}^{k}(M)$,

$$
\left\|f^{s}\right\|_{L^{2}(M)} \leq C\left\|N_{g} f\right\|_{\widetilde{H}^{2}\left(M_{1}\right)}, \quad \forall f \in H^{1}(M),
$$

with a constant $C>0$ that can be chosen locally uniform in $\mathcal{G}^{k}(M)$ in the $C^{k}(M)$ topology.

Of course, $\mathcal{G}^{k}(M)$ includes all real analytic simple metrics in $M$, according to Theorem 2.15.

Sketch of the proof The proof of the basic estimate (99) is based on the following ideas. For $g$ of finite smoothness, one can still construct a parametrix $Q_{g}$ of $N_{g}$ as above that allows us to reconstruct $f^{s}$ from $N_{g} f$ up to smoothing operator terms. This is done in a way similar to that in [179] in two steps: first we invert $N_{g}$ modulo smoothing operators in a neighborhood $M_{1}$ of $M$, and that gives us $f_{M_{1}}^{s}$, i.e., the solenoidal projection of $f$ but associated to the manifold $M_{1}$. Next, we compare $f_{M_{1}}^{s}$ 
and $f^{s}$ and show that one can get the latter from the former by an operator that loses one derivative. This is the same construction as in the proof of Theorem 2.15 above but the metric is only $C^{k}, k \gg 1$. After applying the parametrix $Q_{g}$, the equation for recovering $f^{s}$ from $N_{g} f$ is reduced to solving the Fredholm equation

$$
\left(\mathcal{S}_{g}+K_{g}\right) f=Q_{g} N_{g} f, \quad f \in \mathcal{S}_{g} L^{2}(M)
$$

where $\mathcal{S}_{g}$ is the projection to solenoidal tensors, similarly we denote by $\mathcal{P}_{g}$ the projection onto potential tensors. Here, $K_{g}$ is a compact operator on $\mathcal{S}_{g} L^{2}(M)$. We can write this as an equation in the whole $L^{2}(M)$ by adding $\mathcal{P}_{g} f$ to both sides above to get

$$
\left(I+K_{g}\right) f=\left(Q_{g} N_{g}+\mathcal{P}_{g}\right) f
$$

Then the solenoidal projection of the solution of (101) solves (100). A finite rank modification of $K_{g}$ above can guarantee that $I+K_{g}$ has a trivial kernel, and therefore is invertible, if and only if $N_{g}$ is s-injective. The problem then reduces to that of invertibility of $I+K_{g}$. The operators above depend continuously on $g \in C^{k}, k \gg 1$. Since for $g$ analytic, $I+K_{g}$ is invertible by Theorem 2.15, it would still be invertible in a neighborhood of any analytic $g$, and estimate (99) is true with a locally uniform constant. Analytic (simple) metrics are dense in the set of all simple metrics, and this completes the sketch of the proof of Theorem 2.16. For more details see [181].

The analysis of $I_{g}$ can also be carried out for symmetric tensors of any order, see e.g., $[167,169]$. Since we are motivated by the boundary rigidity problem, and to simplify the exposition, we study only tensors of order 2 . Theorem 2.16 and especially estimate (99) allow us to prove the following local generic uniqueness result for the non-linear boundary rigidity problem.

Theorem 2.17 Let $k_{0}$ and $\mathcal{G}^{k}(M)$ be as in Theorem 2.16. There exists $k \geq k_{0}$, such that for any $g_{0} \in \mathcal{G}^{k}$, there is $\varepsilon>0$, such that for any two metrics $g_{1}, g_{2}$ with $\left\|g_{m}-g_{0}\right\|_{C^{k}(M)} \leq \varepsilon, m=1,2$, we have the following:

$$
d_{g_{1}}=d_{g_{2}} \text { on }(\partial M)^{2} \text { implies } g_{2}=\psi_{*} g_{1}
$$

with some $C^{k+1}(M)$-diffeomorphism $\psi: M \rightarrow M$ fixing the boundary.

Sketch of the proof We prove Theorem 2.17 by linearizing and using Theorem 2.16, and especially (99), see also [179]. This requires first to pass to special semigeodesic coordinates related to each metric in which $g_{i n}=\delta_{i n}, \forall i$. We denote the corresponding pull-backs by $g_{1}, g_{2}$ again. Then we show that if $g_{1}$ and $g_{2}$ have the same distance on the boundary, then $g_{1}=g_{2}$ on the boundary with all derivatives. As a result, for $f:=g_{1}-g_{2}$ we get that $f \in C_{0}^{l}(\bar{\Omega})$ with $l \gg 1$, if $k \gg 1$; and $f_{\text {in }}=0, \forall i$. Then we linearize to get

$$
\left\|N_{g_{1}} f\right\|_{L^{\infty}\left(\Omega_{1}\right)} \leq C\|f\|_{C^{1}}^{2},
$$


where $\Omega_{1} \supset \bar{\Omega}$ is as above. Combine this with (99) and interpolation estimates, to get $\forall \mu<1$,

$$
\left\|f^{s}\right\|_{L^{2}} \leq C\|f\|_{L^{2}}^{1+\mu}
$$

One can show that tensors satisfying $f_{i n}=0$ also satisfy $\|f\|_{L^{2}} \leq C\left\|f^{s}\right\|_{H^{2}}$, and using this, and interpolation again, we get

$$
\|f\|_{L^{2}} \leq C\|f\|_{L^{2}}^{1+\mu^{\prime}}, \quad \mu^{\prime}>0
$$

This implies $f=0$ for $\|f\| \ll 1$. Note that the condition $f \in C_{0}^{l}(\bar{\Omega})$ is used to make sure that $f$, extended as zero in $\Omega_{1} \backslash \Omega$, is in $H_{0}^{l}(\Omega)$, and then use this fact in the interpolation estimates. Again, for more details see [181].

Finally, in [181] it is proven a conditional stability estimate of Hölder type. A similar estimate near the Euclidean metric was proven in [206] based on the approach in [178].

Theorem 2.18 Let $k_{0}$ and $\mathcal{G}^{k}(M)$ be as in Theorem 2.16. Then for any $\mu<1$, there exits $k \geq k_{0}$ such that for any $g_{0} \in \mathcal{G}^{k}$, there is an $\varepsilon_{0}>0$ and $C>0$ with the property that for any two metrics $g_{1}, g_{2}$ with $\left\|g_{m}-g_{0}\right\|_{C(M)} \leq \varepsilon_{0}$, and $\left\|g_{m}\right\|_{C^{k}(M)} \leq A$, $m=1,2$, with some $A>0$, we have the following stability estimate

$$
\left\|g_{2}-\psi_{*} g_{1}\right\|_{C^{2}(M)} \leq C(A)\left\|d_{g_{1}}-d_{g_{2}}\right\|_{C(\partial M \times \partial M)}^{\mu}
$$

with some diffeomorphism $\psi: M \rightarrow M$ fixing the boundary.

Sketch of the proof To prove Theorem 2.18, we basically follow the uniqueness proof sketched above by showing that each step is stable. The analysis is more delicate near pairs of points too close to each other. An important ingredient of the proof is stability at the boundary, that is also of independent interest:

Theorem 2.19 Let $g_{0}$ and $g_{1}$ be two simple metrics in $\Omega$, and $\Gamma \subset \subset \Gamma^{\prime} \subset \partial \Omega$ be two sufficiently small open subsets of the boundary. Then for some diffeomorphism $\psi$ fixing the boundary,

$$
\left\|\partial_{x^{n}}^{k}\left(\psi_{*} g_{1}-g_{0}\right)\right\|_{C^{m}(\bar{\Gamma})} \leq C_{k, m}\left\|d_{g_{1}}^{2}-d_{g_{0}}^{2}\right\|_{C^{m+2 k+2}\left(\overline{\Gamma^{\prime} \times \Gamma^{\prime}}\right)}
$$

where $C_{k, m}$ depends only on $\Omega$ and on an upper bound of $g_{0}, g_{1}$ in $C^{m+2 k+5}(\bar{\Omega})$. 
Theorem 2.18 can be used to obtain stability near generic simple metrics for the inverse problem of recovering $g$ from the hyperbolic Dirichlet-to-Neumann map $\Lambda_{g}^{h}$. It is known that $g$ can be recovered uniquely from $\Lambda_{g}$, up to a diffeomorphism as above, see e.g. [24]. This result however relies on a unique continuation theorem by Tataru [194] and it is unlikely to provide Hölder type of stability estimate as above. By using the fact that $d_{g}$ is related to the leading singularities in the kernel of $\Lambda_{g}^{h}$, it was proven a Hölder stability estimate under the assumptions above, relating $g$ and $\Lambda_{g}$. We refer to [180] for details.

\subsection{Lens rigidity}

For non-simple manifolds in particular, if we have conjugate points or the boundary is not strictly convex, we need to look at the behavior of all the geodesics and the scattering relation encodes this information. We proceed to define in more detail the scattering relation for non-convex manifolds and the lens rigidity problem and state our results. We note that we will also consider the case of incomplete data, that is when we don't have information about all the geodesics entering the manifold. More details can be found in $[182,183]$.

The scattering relation

$$
\Sigma: \partial_{+} S M \rightarrow \overline{\partial_{-} S M}
$$

is defined by $\Sigma(x, \xi)=(y, \eta)=\Phi^{\ell}(x, \xi)$, where $\Phi^{t}$ is the geodesic flow, and $\ell>0$ is the first moment, at which the unit speed geodesic through $(x, \xi)$ hits $\partial M$ again. If such an $\ell$ does not exists, we formally set $\ell=\infty$ and we call the corresponding initial condition and the corresponding geodesic trapping. This defines also $\ell(x, \xi)$ as a function $\ell: \partial_{+} S M \rightarrow[0, \infty]$. Note that $\Sigma$ and $\ell$ are not necessarily continuous. This coincides with the scattering relation $\alpha$ defined in Sect. 2.2 for strictly convex manifolds.

It is convenient to think of $\Sigma$ and $\ell$ as defined on the whole $\partial S M$ with $\Sigma=I d$ and $\ell=0$ on $\overline{\partial_{-} S M}$. We parametrize the scattering relation in a way that makes it independent of pulling it back by diffeomorphisms fixing $\partial M$ pointwise. Let $\kappa_{ \pm}$: $\partial_{ \pm} S M \rightarrow B(\partial M)$ be the orthogonal projection onto the (open) unit ball tangent bundle that extends continuously to the closure of $\partial_{ \pm} S M$. Then $\kappa_{ \pm}$are homeomorphisms, and we set

$$
\sigma=\kappa_{+} \circ \Sigma \circ \kappa_{-}^{-1}: \overline{B(\partial M)} \longrightarrow \overline{B(\partial M)}
$$

According to our convention, $\sigma=I d$ on $\partial(\overline{B(\partial M)})=S(\partial M)$. We equip $\overline{B(\partial M)}$ with the relative topology induced by $T(\partial M)$, where neighborhoods of boundary points (those in $S(\partial M)$ ) are given by half-neighborhoods, i.e., by neighborhoods in $T(\partial M)$ intersected with $\overline{B(\partial M)}$. It is possible to define $\sigma$ in a way that does not require knowledge of $\left.g\right|_{T(\partial M)}$ by thinking of any boundary vector $\xi$ as characterized by its angle with $\partial M$ and the direction of its tangential projection. Let $\mathcal{D}$ be an open subset of $\overline{B(\partial M)}$. A priori, the latter depends on $\left.g\right|_{T(\partial M)}$. By the remark above, we can think 
of it as independent of $\left.g\right|_{T(\partial M)}$ however. The lens rigidity problem we study is the following: Given $M$ and $\mathcal{D}$, do $\sigma$ and $\ell$, restricted to $\mathcal{D}$, determine $g$ uniquely, up to a pull back of a diffeomorphism that is identity on $\partial M$ ? The answer to this question, even when $\mathcal{D}=B(\partial M)$, is negative, see [48]. The known counter-examples are trapping manifolds. The boundary rigidity problem and the lens rigidity one are equivalent for simple metrics.

\subsection{Main assumptions}

Definition 2.20 We say that $\mathcal{D}$ is complete for the metric $g$, if for any $(z, \zeta) \in T^{*} M$ there exists a maximal in $M$, finite length unit speed geodesic $\gamma:[0, l] \rightarrow M$ through $z$, normal to $\zeta$, such that

$$
\{(\gamma(t), \dot{\gamma}(t)) ; 0 \leq t \leq l\} \cap S(\partial M) \subset \mathcal{D},
$$

there are no conjugate points on $\gamma$.

We call the $C^{k}$ metric $g$ regular, if a complete set $\mathcal{D}$ exists, i.e., if $\overline{B(\partial M)}$ is complete.

If $z \in \partial M$ and $\zeta$ is conormal to $\partial M$, then $\gamma$ may reduce to one point.

Topological Condition (T): Any path in $M$ connecting two boundary points is homotopic to a polygon $c_{1} \cup \gamma_{1} \cup c_{2} \cup \gamma_{2} \cup \cdots \cup \gamma_{k} \cup c_{k+1}$ with the properties that for any $j$, (i) $c_{j}$ is a path on $\partial M$; (ii) $\gamma_{j}:\left[0, l_{j}\right] \rightarrow M$ is a geodesic lying in $M^{\text {int }}$ with the exception of its endpoints and is transversal to $\partial M$ at both ends; moreover, $\kappa_{-}\left(\gamma_{j}(0), \dot{\gamma}_{j}(0)\right) \in \mathcal{D}$; Notice that $(\mathrm{T})$ is an open condition w.r.t. $g$, i.e., it is preserved under small $C^{2}$ perturbations of $g$. To define the $C^{K}(M)$ norm below in a unique way, we choose and fix a finite atlas on $M$.

\subsubsection{Results about the linear problem}

We refer to [182] for more details about the results in this section. It turns out that a linearization of the lens rigidity problem is again the problem of s-injectivity of the ray transform $I$. Here and below we sometimes drop the subscript $g$. Given $\mathcal{D}$ as above, we denote by $I_{\mathcal{D}}\left(\right.$ or $I_{g, \mathcal{D}}$ ) the ray transform $I$ restricted to the maximal geodesics issued from $(x, \xi) \in \kappa_{-}^{-1}(\mathcal{D})$. The first result of this section generalizes Theorem 2.15.

Theorem 2.21 Let $g$ be an analytic, regular metric on $M$. Let $\mathcal{D}$ be complete and open. Then $I_{\mathcal{D}}$ is s-injective.

Sketch of the proof Since we know integrals over a subset of geodesics only, this creates difficulties with cut-offs in the phase variable that cannot be analytic. For this reason, the proof of Theorem 2.21 is different from that of Theorem 2.15. Let $g$ be an analytic regular metrics in $M$, and let $M_{1} \ni M$ be the manifold where $g$ is extended analytically. There is an analytic atlas in $M$, and $\partial M$ can be assumed to be analytic, too. In other words, now $(M, \partial M, g)$ is a real analytic manifold with boundary. We denote by $\mathcal{A}(M)$ (respectively $\mathcal{A}\left(M_{1}\right)$ ) the set of analytic functions on $M$ (respectively 
$\left.M_{1}\right)$. Next, $f_{M_{1}}^{s}$ denotes the solenoidal part of the tensor $f$, extended as zero to $M_{1}$, in the manifold $M_{1}$. The main step is to show that $I_{\mathcal{D}} f=0$ implies $f^{s} \in \mathcal{A}(M)$. In order to do that one shows that $f_{M_{1}}^{s} \in \mathcal{A}\left(M_{1}\right)$. Let us first notice, that in $M_{1} \backslash M$, $f_{M_{1}}^{s}=-d v_{M_{1}}$, where $v_{M_{1}}$ satisfies $\delta d v_{M_{1}}=0$ in $M_{1} \backslash M,\left.v\right|_{\partial M_{1}}=0$ since $f=0$ in $M_{1} \backslash M$. Therefore, $v_{M_{1}}$ is analytic up to $\partial M_{1}$. Therefore, we only need to show that $f_{M_{1}}^{s}$ is analytic in the interior of $M_{1}$. Below, $\mathrm{WF}_{\mathrm{A}}(f)$ stands for the analytic wave front set of $f$, see $[173,195]$. The crucial point is the following microlocal analytic regularity result.

Proposition 2.22 Let $\gamma_{0}$ be a fixed maximal geodesic in $M$ with endpoints on $\partial M$, without conjugate points, and let $I_{g} f(\gamma)=0$ for $\gamma \in \operatorname{neigh}\left(\gamma_{0}\right)$. Let $g$ be analytic in $\operatorname{neigh}\left(\gamma_{0}\right)$. Then

$$
N^{*} \gamma_{0} \cap \mathrm{WF}_{\mathrm{A}}\left(f_{M_{1}}^{s}\right)=\emptyset
$$

Sketch of the proof Set $f=f_{M_{1}}^{s}$. Let $U_{\varepsilon}$ be a tubular neighborhood of $\gamma_{0}$, and $x=$ $\left(x^{\prime}, x^{n}\right)$ be semigeodesic coordinates in it such that $x^{\prime}=0$ on $\gamma_{0}$. Fix $x_{0} \in \gamma_{0} \cap M$. We can assume that $x_{0}=0$ and $g_{i j}(0)=\delta_{i j}$. Then we can assume that $U_{\varepsilon}=\left\{-l_{1}-\varepsilon<\right.$ $\left.x^{n}<l_{2}+\varepsilon,\left|x^{\prime}\right|<\varepsilon\right\}$ with the part of $\gamma_{0}$ corresponding to $x^{n} \notin\left[-l_{1}, l_{2}\right]$ outside $M$. Fix $\xi^{0}=\left(\left(\xi^{0}\right)^{\prime}, 0\right)$ with $\xi_{n}^{0}=0$. We will show that

$$
\left(0, \xi^{0}\right) \notin \mathrm{WF}_{\mathrm{A}}(f) .
$$

We choose a local chart for the geodesics close to $\gamma_{0}$. Set first $Z=\left\{x^{n}=0 ; \quad\left|x^{\prime}\right|<\right.$ $7 \varepsilon / 8\}$, and denote the $x^{\prime}$ variable on $Z$ by $z^{\prime}$. Then $z^{\prime}, \theta^{\prime}$ (with $\left|\theta^{\prime}\right| \ll 1$ ) are local coordinates in neigh $\left(\gamma_{0}\right)$ determined by $\left(z^{\prime}, \theta^{\prime}\right) \rightarrow \gamma_{\left(z^{\prime}, 0\right),\left(\theta^{\prime}, 1\right)}$ where the latter denotes the geodesic through the point $\left(z^{\prime}, 0\right)$ in the direction $\left(\theta^{\prime}, 1\right)$. Let $\chi_{N}\left(z^{\prime}\right)$ be a smooth cut-off function equal to 1 for $\left|z^{\prime}\right| \leq 3 \varepsilon / 4$ and supported in $Z$, also satisfying $\left|\partial^{\alpha} \chi_{N}\right| \leq$ $(C N)^{|\alpha|},|\alpha| \leq N$. Set $\theta=\left(\theta^{\prime}, 1\right),\left|\theta^{\prime}\right| \ll 1$, and multiply

$$
I f\left(\gamma_{\left(z^{\prime}, 0\right), \theta}\right)=0
$$

by $\chi_{N}\left(z^{\prime}\right) e^{\mathrm{i} \lambda z^{\prime} \cdot \xi^{\prime}}$, where $\lambda>0, \xi^{\prime}$ is in a complex neighborhood of $\left(\xi^{0}\right)^{\prime}$, and integrate w.r.t. $z^{\prime}$ to get

$$
\iint e^{\mathrm{i} \lambda z^{\prime} \cdot \xi^{\prime}} \chi_{N}\left(z^{\prime}\right) f_{i j}\left(\gamma_{\left(z^{\prime}, 0\right), \theta}(t)\right) \dot{\gamma}_{\left(z^{\prime}, 0\right), \theta}^{i}(t) \dot{\gamma}_{\left(z^{\prime}, 0\right), \theta}^{j}(t) \mathrm{d} t \mathrm{~d} z^{\prime}=0 .
$$

Set $x=\gamma_{\left(z^{\prime}, 0\right), \theta}(t)$. If $\theta^{\prime}=0$, we have $x=\left(z^{\prime}, t\right)$. By a perturbation argument, for $\theta^{\prime}$ fixed and small enough, $\left(t, z^{\prime}\right)$ are analytic local coordinates, depending analytically on $\theta^{\prime}$. In particular, $x=\left(z^{\prime}+t \theta^{\prime}, t\right)+O\left(\left|\theta^{\prime}\right|\right)$ but this expansion is not enough for the analysis below. Performing a change of variables in (109), we get

$$
\int e^{\mathrm{i} \lambda z^{\prime}\left(x, \theta^{\prime}\right) \cdot \xi^{\prime}} a_{N}\left(x, \theta^{\prime}\right) f_{i j}(x) b^{i}\left(x, \theta^{\prime}\right) b^{j}\left(x, \theta^{\prime}\right) \mathrm{d} x=0
$$

for $\left|\theta^{\prime}\right| \ll 1, \forall \lambda, \forall \xi^{\prime}$, where, for $\left|\theta^{\prime}\right| \ll 1$, the function $\left(x, \theta^{\prime}\right) \mapsto a_{N}$ is positive for $x$ in a neighborhood of $\gamma_{0}$, vanishing for $x \notin U_{\varepsilon}$, and satisfies the same estimate as 
$\chi_{N}$. The vector field $b$ is analytic, and $b\left(0, \theta^{\prime}\right)=\theta, a_{N}\left(0, \theta^{\prime}\right)=1$. To clarify the approach, note that if $g$ is Euclidean in neigh $\left(\gamma_{0}\right)$, then $(110)$ reduces to

$$
\int e^{\mathrm{i} \lambda\left(\xi^{\prime},-\theta^{\prime} \cdot \xi^{\prime}\right) \cdot x} \chi f_{i j}(x) \theta^{i} \theta^{j} \mathrm{~d} x=0,
$$

where $\chi=\chi\left(x^{\prime}-x^{n} \theta^{\prime}\right)$. Then $\xi=\left(\xi^{\prime},-\theta^{\prime} \cdot \xi^{\prime}\right)$ is perpendicular to $\theta=\left(\theta^{\prime}, 1\right)$. This implies that

$$
\int e^{\mathrm{i} \lambda \xi \cdot x} \chi f_{i j}(x) \theta^{i}(\xi) \theta^{j}(\xi) \mathrm{d} x=0
$$

for any function $\theta(\xi)$ defined near $\xi^{0}$, such that $\theta(\xi) \cdot \xi=0$. This has been noticed and used before if $g$ is close to the Euclidean metric (with $\chi=1$ ), see e.g., [178]. We will assume that $\theta(\xi)$ is analytic. A simple argument (see e.g. [167,178]) shows that a constant symmetric tensor $f_{i j}$ is uniquely determined by the numbers $f_{i j} \theta^{i} \theta^{j}$ for finitely many $\theta^{\prime}$ 's (actually, for $N^{\prime}=(n+1) n / 2 \theta$ 's); and in any open set on the unit sphere, there are such $\theta^{\prime}$ 's. On the other hand, $f$ is solenoidal. To simplify the argument, assume for a moment that $f$ vanishes on $\partial M$. Then $\xi^{i} \widehat{f}_{i j}(\xi)=0$. Therefore, combining this with (111), we need to choose $N=n(n-1) / 2$ vectors $\theta(\xi)$, perpendicular to $\xi$, that would uniquely determine the tensor $\widehat{f}$ on the plane perpendicular to $\xi$. To this end, it is enough to know that this choice can be made for $\xi=\xi^{0}$, then it would be true for $\xi \in \operatorname{neigh}\left(\xi^{0}\right)$. This way, $\xi^{i} \widehat{f}_{i j}(\xi)=0$ and the $N$ Eqs. (111) with the so chosen $\theta_{p}(\xi), p=1, \ldots, N$, form a system with a tensor-valued symbol elliptic near $\xi=\xi^{0}$. The $C^{\infty} \Psi$ DOcalculus easily implies the statement of the lemma in the $C^{\infty}$ category, and the complex stationary phase method below, or the analytic $\Psi$ DOcalculus in [195] with appropriate cut-offs in $\xi$, implies the lemma in this special case ( $g$ locally Euclidean). The general case is considered in [182], and is based on an application of a complex stationary phase argument [173] to (110) as in [107].

Proposition 2.22 makes it possible to prove that $f^{s} \in \mathcal{A}(M)$. We combine this with a boundary determination theorem for tensors, a linear version of Theorem 2.28 below, to conclude that then $f=0$.

Next, we formulate a stability estimate in the spirit of Theorem 2.16. We need first to parametrize (a complete subset of) the geodesics issued from $\mathcal{D}$ in a different way that would make them a manifold. The parametrization provided by $\mathcal{D}$ is inconvenient near the directions tangent to $\partial M$. Let $H_{m}$ be a finite collection of smooth hypersurfaces in $M_{1}^{\text {int }}$. Let $\mathcal{H}_{m}$ be an open subset of $\left\{(z, \theta) \in S M_{1} ; z \in H_{m}, \theta \notin T_{z} H_{m}\right\}$, and let $\pm l_{m}^{ \pm}(z, \theta) \geq 0$ be two continuous functions. Let $\Gamma\left(\mathcal{H}_{m}\right)$ be the set of geodesics

$$
\Gamma\left(\mathcal{H}_{m}\right)=\left\{\gamma_{z, \theta}(t) ; l_{m}^{-}(z, \theta) \leq t \leq l_{m}^{+}(z, \theta),(z, \theta) \in \mathcal{H}_{m}\right\}
$$

that, depending on the context, is considered either as a family of curves, or as a point set. We also assume that each $\gamma \in \Gamma\left(\mathcal{H}_{m}\right)$ is a simple geodesic (no conjugate points). If $g$ is simple, then one can take a single $H=\partial M_{1}$ with $l^{-}=0$ and an 
appropriate $l^{+}(z, \theta)$. If $g$ is regular only, and $\Gamma$ is any complete set of geodesics, then any small enough neighborhood of a simple geodesic in $\Gamma$ has the properties listed in the paragraph above and by a compactness argument one can choose a finite complete set of such $\Gamma\left(\mathcal{H}_{m}\right)$ 's, that is included in the original $\Gamma$. Given $\mathcal{H}=\left\{\mathcal{H}_{m}\right\}$ as above, we consider an open set $\mathcal{H}^{\prime}=\left\{\mathcal{H}_{m}^{\prime}\right\}$, such that $\mathcal{H}_{m}^{\prime} \Subset \mathcal{H}_{m}$, and let $\Gamma\left(\mathcal{H}_{m}^{\prime}\right)$ be the associated set of geodesics defined as in (112), with the same $l_{m}^{ \pm}$. Set $\Gamma(\mathcal{H})=$ $\cup \Gamma\left(\mathcal{H}_{m}\right), \Gamma\left(\mathcal{H}^{\prime}\right)=\cup \Gamma\left(\mathcal{H}_{m}^{\prime}\right)$. The restriction $\gamma \in \Gamma\left(\mathcal{H}_{m}^{\prime}\right) \subset \Gamma\left(\mathcal{H}_{m}\right)$ can be modeled by introducing a weight function $\alpha_{m}$ in $\mathcal{H}_{m}$, such that $\alpha_{m}=1$ on $\mathcal{H}_{m}^{\prime}$, and $\alpha_{m}=0$ otherwise. More generally, we allow $\alpha_{m}$ to be smooth but still supported in $\mathcal{H}_{m}$. We then write $\alpha=\left\{\alpha_{m}\right\}$, and we say that $\alpha \in C^{k}(\mathcal{H})$, if $\alpha_{m} \in C^{k}\left(\mathcal{H}_{m}\right), \forall m$. We consider $I_{\alpha_{m}}=\alpha_{m} I$, or more precisely, in the coordinates $(z, \theta) \in \mathcal{H}_{m}$,

$$
I_{\alpha_{m}} f=\alpha_{m}(z, \theta) \int_{0}^{l_{m}(z, \theta)}\left\langle f\left(\gamma_{z, \theta}\right), \dot{\gamma}_{z, \theta}^{2}\right\rangle \mathrm{d} t, \quad(z, \theta) \in \mathcal{H}_{m} .
$$

Next, we set

$$
I_{\alpha}=\left\{I_{\alpha_{m}}\right\}, \quad N_{\alpha_{m}}=I_{\alpha_{m}}^{*} I_{\alpha_{m}}=I^{*}\left|\alpha_{m}\right|^{2} I, \quad N_{\alpha}=\sum N_{\alpha_{m}}
$$

where the adjoint is taken w.r.t. the measure $\mathrm{d} \mu:=|\langle\nu(z), \theta\rangle| \mathrm{d} S_{z} \mathrm{~d} \theta$ on $\mathcal{H}_{m}, \mathrm{~d} S_{z} \mathrm{~d} \theta$ being the induced measure on $S M$, and $v(z)$ being a unit normal to $H_{m}$. S-injectivity of $N_{\alpha}$ is equivalent to s-injectivity for $I_{\alpha}$, which in turn is equivalent to s-injectivity of $I$ restricted to $\operatorname{supp} \alpha$.

Theorem 2.23 (a) Let $g=g_{0} \in C^{k}, k \gg 1$ be regular, and let $\mathcal{H}^{\prime} \Subset \mathcal{H}$ be as above with $\Gamma\left(\mathcal{H}^{\prime}\right)$ complete. Fix $\alpha=\left\{\alpha_{m}\right\} \in C^{\infty}$ with $\mathcal{H}_{m}^{\prime} \subset$ supp $\alpha_{m} \subset \mathcal{H}_{m}$. Then if $I_{\alpha}$ is s-injective, we have

$$
\left\|f^{s}\right\|_{L^{2}(M)} \leq C\left\|N_{\alpha} f\right\|_{\widetilde{H}^{2}\left(M_{1}\right)} .
$$

(b) Assume that $\alpha=\alpha_{g}$ in (a) depends on $g \in C^{k}$, so that $C^{k}\left(M_{1}\right) \ni g \rightarrow C^{l}(\mathcal{H}) \ni$ $\alpha_{g}$ is continuous with $l \gg 1, k \gg 1$. Assume that $I_{g_{0}, \alpha_{g_{0}}}$ is s-injective. Then estimate (115) remains true for $g$ in a small enough neighborhood of $g_{0}$ in $C^{k}\left(M_{1}\right)$ with a uniform constant $C>0$.

The theorem above allows us to formulate a generic result:

Theorem 2.24 Let $\mathcal{G} \subset C^{k}(M)$ be an open set of regular Riemannian metrics on $M$ such that $(T)$ is satisfied for each one of them. Let the set $\mathcal{D}^{\prime} \subset \partial S M$ be open and complete for each $g \in \mathcal{G}$. Then there exists an open and dense subset $\mathcal{G}_{s}$ of $\mathcal{G}$ such that $I_{g, \mathcal{D}^{\prime}}$ is s-injective for any $g \in \mathcal{G}_{s}$.

Of course, the set $\mathcal{G}_{s}$ includes all real analytic metrics in $\mathcal{G}$.

Corollary 2.25 Let $\mathcal{R}(M)$ be the set of all regular $C^{k}$ metrics on $M$ satisfying $(T)$ equipped with the $C^{k}\left(M_{1}\right)$ topology. Then for $k \gg 1$, the subset of metrics for which the X-ray transform I over all simple geodesics through all points in $M$ is s-injective, is open and dense in $\mathcal{R}(M)$. 
2.6 Results about the non-linear lens rigidity problem

Using the results above, we prove the following about the lens rigidity problem on manifolds satisfying the assumptions in Sect. 2.5. More details can be found in [183]. Theorem 2.26 below says, loosely speaking, that for the classes of manifolds and metrics we study, the uniqueness question for the non-linear lens rigidity problem can be answered locally by linearization. This is a non-trivial implicit function type of theorem however because our success heavily depends on the a priori stability estimate that the s-injectivity of $I_{\mathcal{D}}$ implies; see Theorem 2.23; and the latter is based on the hypoelliptic properties of $I_{\mathcal{D}}$. We work with two metrics $g$ and $\widehat{g}$; and will denote objects related to $\widehat{g}$ by $\widehat{\sigma}, \widehat{\ell}$, etc.

Theorem 2.26 Let $\left(M, g_{0}\right)$ satisfy the topological assumption $(T)$, with $g_{0} \in C^{k}(M)$ a regular Riemannian metric with $k \gg 1$. Let $\mathcal{D}$ be open and complete for $g_{0}$, and assume that there exists $\mathcal{D}^{\prime} \Subset \mathcal{D}$ so that $I_{g_{0}} \mathcal{D}^{\prime}$ is s-injective. Then there exists $\varepsilon>0$, such that for any two metrics $g, \widehat{g}$ satisfying

$$
\left\|g-g_{0}\right\|_{C^{k}(M)}+\left\|\widehat{g}-g_{0}\right\|_{C^{k}(M)} \leq \varepsilon
$$

the relations

$$
\sigma=\widehat{\sigma}, \quad \ell=\widehat{\ell} \text { on } \mathcal{D}
$$

imply that there is a $C^{k+1}$ diffeomorphism $\psi: M \rightarrow M$ fixing the boundary such that

$$
\widehat{g}=\psi^{*} g
$$

By Theorem 2.24, the requirement that $I_{g_{0}, \mathcal{D}^{\prime}}$ is s-injective is a generic one for $g_{0}$. Therefore, Theorems 2.26 and 2.24 combined imply that there is local uniqueness, up to isometry, near a generic set of regular metrics.

Corollary 2.27 Let $\mathcal{D}^{\prime} \Subset \mathcal{D}, \mathcal{G}, \mathcal{G}_{s}$ be as in Theorem 2.24. Then the conclusion of Theorem 2.26 holds for any $g_{0} \in \mathcal{G}_{s}$.

\subsubsection{Boundary determination of the jet of $g$}

The first step of the proof of Theorem 2.26 is to determine all derivatives of $g$ on $\partial M$. The following theorem is interesting by itself. Notice that $g$ below does not need to be analytic or generic.

Theorem 2.28 Let $(M, g)$ be a compact Riemannian manifold with boundary. Let $\left(x_{0}, \xi_{0}\right) \in S(\partial M)$ be such that the maximal geodesic $\gamma_{x_{0}, \xi_{0}}$ through it is of finite length, and assume that $x_{0}$ is not conjugate to any point in $\gamma_{x_{0}}, \xi_{0} \cap \partial M$. If $\sigma$ and $\ell$ are known on some neighborhood of $\left(x_{0}, \xi_{0}\right)$, then the jet of $g$ at $x_{0}$ in boundary normal coordinates is determined uniquely. 
Sketch of the proof of Theorem 2.28 To make the arguments below more transparent, assume that the geodesic $\gamma_{0}$ issued from $\left(x_{0}, \xi_{0}\right)$ hits $\partial M$ for the first time transversally at $\gamma_{0}\left(l_{0}\right)=y_{0}, l_{0}>0$. Then $y_{0}$ is the only point on $\partial M$ reachable from $\left(x_{0}, \xi_{0}\right)$, and $x_{0}$, $y_{0}$ are not conjugate points on $\gamma_{0}$ by assumption. Assume also that $\gamma_{0}$ is tangent of finite order at $x_{0}$. Then there is a half neighborhood $V$ of $x_{0}$ on $\partial M$ visible from $y_{0}$. The latter is not always true if $\gamma_{0}$ is tangent to $\partial M$ of infinite order at $x_{0}$. Choose local boundary normal coordinates near $x_{0}$ and $y_{0}$, and let $g_{0}$ be the Euclidean metric in each of them w.r.t. to the so chosen coordinates. We can then consider a representation of $\Sigma$, denoted by $\Sigma^{\sharp}$ below, defined locally on $\mathbb{R}^{n-1} \times S^{n-1}$, with values on another copy of the same space. If $(x, \theta) \in \mathbb{R}^{n-1} \times S^{n-1}$, then the associated vector at $x \in \partial M$ is $\xi=\theta /|\theta|_{g}$; and $\Sigma^{\sharp}(x, \theta)=\Sigma(x, \xi)$. The same applies to the second component of $\Sigma^{\sharp}(x, \theta)$. Namely, if $(y, \eta)=\Sigma(x, \xi)$, then we set $\omega=\eta /|\eta|_{g_{0}}$, then $\Sigma^{\sharp}:(x, \theta) \mapsto(y, \omega)$. Similarly, we set $\ell^{\sharp}(x, \theta)=\ell(x, \xi)$. Let also $\theta_{0}$ and $\omega_{0}$ correspond to $\xi_{0}$ and $\eta_{0}$, respectively, where $\Sigma\left(x_{0}, \xi_{0}\right)=\left(y_{0}, \eta_{0}\right)$. Set $\tau(x):=\tau\left(x, y_{0}\right)$, where $\tau$ is the smooth travel time function localized near $x=x_{0}$ such that $\tau\left(x_{0}, y_{0}\right)=l_{0}$. Then $\tau$ is well defined in a small neighborhood of $x_{0}$ by the implicit function theorem and the assumption that $x_{0}$ and $y_{0}$ are not conjugate on $\gamma_{0}$. In the normal boundary coordinates $x=\left(x^{\prime}, x^{n}\right)$ near $x_{0}, g_{i n}=\delta_{i n}, \forall i$. Since $x_{0}$ and $y_{0}$ are not conjugate, for $\eta \in S_{y_{0}} M$ close enough to $\eta_{0}$, the map $\eta \mapsto x \in \partial M$ is a local diffeomorphism as long as the geodesic connecting $x$ and $y_{0}$ is not tangent to $\partial M$ at $x$. Moreover, that map is known, being the inverse of $\Sigma$. Similarly, the map $S^{n-1} \ni \omega \mapsto x$ is a local diffeomorphism and is also known. Then we know $(x,-\theta)=\Sigma^{\sharp}\left(y_{0},-\omega\right)$, and we know $\ell^{\sharp}\left(y_{0},-\omega\right)=\ell^{\sharp}(x, \theta)=\tau(x)$. Then we can recover $\operatorname{grad}^{\prime} \tau=-\theta^{\prime} /|\theta|_{g}$, where the prime stands for tangential projection as usual. Taking the limit $\omega \rightarrow \omega_{0}$, we recover $\left|\theta_{0}\right|_{g}^{2}=g_{\alpha \beta} \theta_{0}^{\alpha} \theta_{0}^{\beta}$. We use again the fact that a symmetric $n \times n$ tensor $f_{i j}$ can be recovered by knowledge of $f_{i j} p_{k}^{i} p_{k}^{j}$ for $N=n(n+1) / 2$ "generic" vectors $p_{k}, k=1, \ldots, N$; and such $N$ vectors exist in any open set on $S^{n-1}$, see e.g. [183]. Thus choosing appropriate $n(n-1) / 2$ perturbations of $\theta_{0}$ 's, we recover $g\left(x_{0}\right)$. Thus, we recover $g$ in a neighborhood of $x_{0}$ as well; we can assume that $V$ covers that neighborhood. Note that we know all tangential derivatives of $g$ in $V \ni x_{0}$. Then $\tau$ solves the eikonal equation

$$
g^{\alpha \beta} \tau_{x^{\alpha}} \tau_{x^{\beta}}+\tau_{x^{n}}^{2}=1
$$

Next, in $V$, we know $\tau_{x^{\alpha}}, \alpha \leq n-1$, we know $g$, therefore by (117), we get $\tau_{x^{n}}^{2}$. It is easy to see that $\tau_{x^{n}} \leq 0$ on the visible part, so we recover $\tau_{x^{n}}$ there. We therefore know the tangential derivatives of $\tau_{x^{n}}$ on $\partial M$ near $x_{0}$. Differentiate (117) w.r.t. $x^{n}$ at $x=x_{0}$ to get

$$
\left.\left[\frac{d g^{\alpha \beta}}{d x^{n}} \tau_{x^{\alpha}} \tau_{x^{\beta}}+2 g^{\alpha \beta} \tau_{x^{\alpha} x^{n}} \tau_{x^{\beta}}+2 \tau_{x^{n} x^{n}} \tau_{x^{n}}\right]\right|_{x=x_{0}}=0
$$

Since $\gamma_{0}$ is tangent to $\partial M$ at $x_{0}$, we have $\tau_{x^{n}}\left(x_{0}\right)=0$ by (117). The third term in the 1.h.s. of (118) therefore vanishes. Therefore the only unknown term in (118) is $\gamma^{\alpha \beta}:=d g^{\alpha \beta} / d x^{n}$ at $x=x_{0}$. Since $\tau_{x^{\alpha}}\left(x_{0}\right)=-\xi_{0}$, using the fact that $\operatorname{grad} \tau\left(x_{0}\right)=$ $-\xi_{0}$ again, we get that we have to determine $\gamma^{\alpha \beta}$ from $\gamma_{\alpha \beta} \xi_{0}^{\alpha} \xi_{0}^{\beta}$. This is possible if 
as above, we repeat the construction and replace $\xi_{0}$ by a finite number of vectors, close enough to $\xi_{0}$. So we get an explicit formula for $\partial g /\left.\partial x^{i}\right|_{\partial M}$ in fact. Next, for $x \in V$ but not on $\partial V$, we can recover $\tau_{x^{n} x^{n}}(x)$ by (118) because $\tau_{x^{n}}(x)<0$. By continuity, we recover $\tau_{x^{n} x^{n}}\left(x_{0}\right)$, therefore we know $\tau_{x^{n} x^{n}}$ near $x_{0}$, and all tangential derivatives of the latter. We differentiate (118) w.r.t. $x^{n}$ again, and as above, recover $d^{2} g /\left.d\left(x^{n}\right)^{2}\right|_{\partial M}$ near $x_{0}$. Then we recover $d^{3} \tau / d\left(x^{n}\right)^{3}$, etc. In the general case, we repeat those arguments with $\xi_{0}$ replaced by $\xi_{0}+\varepsilon v$, where $v$ is the interior unit normal, and take the limit $\varepsilon \rightarrow 0$.

Sketch of the proof of Theorem 2.26 We first find suitable metric $\widehat{g}_{1}$ isometric to $\widehat{g}$, and then we show that $\widehat{g}_{1}=g$. First, we can always assume that $g$ and $\widehat{g}$ have the same boundary normal coordinates near $\partial M$. By [47], there is a metric $h$ isometric to $\widehat{g}$ so that $h$ is solenoidal w.r.t. $g$. Moreover, $h=\widehat{g}+O(\varepsilon)$. By a standard argument, by a diffeomorphism that identifies normal coordinates near $\partial M$ for $h$ and $g$, and is identity away from some neighborhood of the boundary, we find a third $\widehat{g}_{1}$ isometric to $h$ (and therefore to $\widehat{g}$ ), so that $\widehat{g}_{1}=\widehat{g}$ near $\partial M$, and $\widehat{g}_{1}=h$ away from some neighborhood of $\partial M$ (and there is a region that $\widehat{g}_{1}$ is neither). Then $\widehat{g}_{1}-h$ is as small as $g-h$, more precisely,

$$
\left\|\widehat{g}_{1}-h\right\|_{C^{k-3}} \leq C\|g-h\|_{C^{k-1}}, \quad k \gg 1 .
$$

Set

$$
f=h-g, \quad \tilde{f}=\widehat{g}_{1}-g .
$$

Estimate (119) implies

$$
\|\widetilde{f}-f\|_{C^{l-3}} \leq C\|f\|_{C^{l-1}}, \quad \forall l \leq k .
$$

By (116), (121),

$$
\|f\|_{C^{k-1}} \leq C \varepsilon, \quad\|\widetilde{f}\|_{C^{k-3}} \leq C \varepsilon
$$

By Theorem 2.28,

$$
\partial^{\alpha} \widetilde{f}=0 \text { on } \partial M \text { for }|\alpha| \leq k-5 \text {. }
$$

It is known [167] that $2 d v$ is the linearization of $\psi_{\tau}^{*} g$ at $\tau=0$, where $\psi_{\tau}$ is a smooth family of diffeomorphisms, and $v=d \psi_{\tau} / d \tau$ at $\tau=0$. Next proposition is therefore a version of Taylor's expansion:

Proposition 2.29 Let $\widehat{g}$ and $g$ be in $C^{k}, k \geq 2$ and isometric, i.e.,

$$
\widehat{g}=\psi^{*} g
$$


for some diffeomorphism $\psi$ fixing $\partial$ M. Set $f=\widehat{g}-g$. Then there exists $v$ vanishing on $\partial$, so that

$$
f=2 d v+f_{2}
$$

and for $g$ belonging to any bounded set $U$ in $C^{k}$, there exists $C(U)>0$, such that

$$
\left\|f_{2}\right\|_{C^{k-2}} \leq C(U)\|\psi-I d\|_{C^{k-1}}^{2}, \quad\|v\|_{C^{k-1}} \leq C(U)\|\psi-I d\|_{C^{k-1}}
$$

We will sketch now the rest of the proof of Theorem 2.26. We apply Proposition 2.29 to $h$ and $\widehat{g}_{1}$ to get

$$
\widetilde{f}=f+2 d v+f_{2}, \quad\left\|f_{2}\right\|_{C^{l-3}} \leq C\|f\|_{C^{l-1}}^{2}, \quad \forall l \leq k .
$$

In other words, $\widetilde{f} s=f$ up to $O\left(\|f\|^{2}\right)$. We can assume that $g$ is extended smoothly on $M_{1} \ni M$. Next, with $g$ extended as above, we extend $\widehat{g}_{1}$ so that $\widehat{g}_{1}=g$ outside $M$. This can be done in a smooth way by Theorem 2.28 . The next step is to reparametrize the scattering relation. We show that one can extend the maximal geodesics of $g$, respectively $\widehat{g}_{1}$, outside $M$ (where $g=\widehat{g}_{1}$ ), and since the two metrics have the same scattering relation and travel times, they will still have the same scattering relation and travel times if we locally push $\partial M$ a bit outside $M$. Then we can arrange that the new pieces of $\partial M$ are transversal to the geodesics close to a fixed one, which provides a smooth parametrization. By a compactness argument, one can do this near finitely many geodesics issued from point on $\mathcal{D}$, and still have a complete set. This puts as in the situation of Theorem 2.23, where the set of geodesics is parametrized by $\alpha=\left\{\alpha_{j}\right\}$. Next, we linearize the energy functional near each geodesic (in our set of data) related to $g$. Using the assumption that $g$ and $\widehat{g}_{1}$ have the same scattering relation and travel times, we deduct

$$
\left\|N_{\alpha_{j}} \tilde{f}\right\|_{L^{\infty}} \leq C\|\widetilde{f}\|_{C^{1}}^{2}, \quad \forall j
$$

Using interpolation inequalities, and the fact that the extension of $\widetilde{f}$ outside $M$ is smooth enough across $\partial M$ as a consequence of the boundary recovery, we get by (125), and (121),

$$
\left\|N_{\alpha} \widetilde{f}\right\|_{\widetilde{H}^{2}\left(M_{1}\right)} \leq C\|\widetilde{f}\|_{C^{3}}^{3 / 2} \leq C^{\prime}\|f\|_{C^{5}}^{3 / 2}
$$

Since $I_{g_{0}}, \mathcal{D}^{\prime}$ is s-injective, so is $N_{\alpha}$, related to $g_{0}$, by the support properties of $\alpha$. Now, since $g$ is close enough to $g_{0}$ with s-injective $N_{\alpha}$ by (116), $N_{\alpha}$ (the one related to $g$ ) is s-injective as well by Theorem 2.23. Therefore, by (126) and (115),

$$
\left\|f^{s}\right\|_{L^{2}(M)} \leq C\left\|N_{\alpha} \widetilde{f}\right\|_{\widetilde{H}^{2}} \leq C^{\prime}\|f\|_{C^{5}}^{3 / 2}
$$


A decisive moment of the proof is that by Proposition 2.29, see (124), $\widetilde{f}^{s}=f+f_{2}^{s}$, the latter being the solenoidal projection of $f_{2}$. Therefore,

$$
\left\|\widetilde{f}^{s}\right\|_{L^{2}(M)} \geq\|f\|_{L^{2}(M)}-C\|f\|_{C^{2}}^{2}
$$

Together with (127), this yields

$$
\|f\|_{L^{2}(M)} \leq C\left(\|f\|_{C^{2}}^{2}+\|f\|_{C^{5}}^{3 / 2}\right) \leq C^{\prime}\|f\|_{C^{5}}^{3 / 2}
$$

because the $C^{5}$ norm of $f$ is uniformly bounded when $\varepsilon \leq 1$. Using interpolation again, we easily deduct $\|f\|_{L^{2}(M)} \geq 1 / C$ if $f \neq 0$. This contradicts (122) if $\varepsilon \ll 1$. Now, $f=0$ implies $h=g$, therefore, $g$ and $\widehat{g}$ are isometric. This concludes the sketch proof of Theorem 2.26.

\section{Invisibility for electrostatics}

We discuss here only invisibility results for electrostatics. For similar results for electromagnetic waves, acoustic waves, quantum waves, etc., see the review papers $[61,62]$ and the references given there. The fact that the boundary measurements do not change, when a conductivity is pushed forward by a smooth diffeomorphism leaving the boundary fixed (see Sect. 1.7), can already be considered as a weak form of invisibility. Different media appear to be the same, and the apparent location of objects can change. However, this does not yet constitute real invisibility, as nothing has been hidden from view. In invisibility cloaking the aim is to hide an object inside a domain by surrounding it with a material so that even the presence of this object can not be detected by measurements on the domain's boundary. This means that all boundary measurements for the domain with this cloaked object included would be the same as if the domain were filled with a homogeneous, isotropic material. Theoretical models for this have been found by applying diffeomorphisms having singularities. These were first introduced in the framework of electrostatics, yielding counterexamples to the anisotropic Calderón problem in the form of singular, anisotropic conductivities in $\mathbb{R}^{n}, n \geq 3$, indistinguishable from a constant isotropic conductivity in that they have the same Dirichlet-to-Neumann map [64,65]. The same construction was rediscovered for electromagnetism in [153], with the intention of actually building such a device with appropriately designed metamaterials; a modified version of this was then experimentally demonstrated in [171]. (See also [126] for a somewhat different approach to cloaking in the high frequency limit.) The first constructions in this direction were based on blowing up the metric around a point [125]. In this construction, let $(M, g)$ be a compact 2-dimensional manifold with non-empty boundary, let $x_{0} \in M$ and consider the manifold

$$
\widetilde{M}=M \backslash\left\{x_{0}\right\}
$$


with the metric

$$
\widetilde{g}_{i j}(x)=\frac{1}{d_{M}\left(x, x_{0}\right)^{2}} g_{i j}(x),
$$

where $d_{M}\left(x, x_{0}\right)$ is the distance between $x$ and $x_{0}$ on $(M, g)$. Then $(\tilde{M}, \widetilde{g})$ is a complete, non-compact 2-dimensional Riemannian manifold with the boundary $\partial \widetilde{M}=\partial M$. Essentially, the point $x_{0}$ has been "pulled to infinity". On the manifolds $M$ and $\widetilde{M}$ we consider the boundary value problems

$$
\left\{\begin{array} { l l } 
{ \Delta _ { g } u = 0 } & { \text { in } M , } \\
{ u = f \quad \text { on } \partial M , }
\end{array} \text { and } \left\{\begin{array}{ll}
\Delta \widetilde{g} \widetilde{u}=0 & \text { in } \widetilde{M}, \\
\widetilde{u}=f & \text { on } \partial \widetilde{M}, \\
\widetilde{u} \in L^{\infty}(\widetilde{M}) . &
\end{array}\right.\right.
$$

These boundary value problems are uniquely solvable and define the DN maps

$$
\Lambda_{M, g} f=\left.\partial_{\nu} u\right|_{\partial M}, \quad \Lambda_{\tilde{M}, \tilde{g}} f=\left.\partial_{\nu} \widetilde{u}\right|_{\partial \widetilde{M}}
$$

where $\partial_{v}$ denotes the corresponding conormal derivatives. Since, in the two dimensional case, functions which are harmonic with respect to the metric $g$ stay harmonic with respect to any metric which is conformal to $g$, one can see that $\Lambda_{M, g}=\Lambda_{\tilde{M}, \tilde{g}}$. This can be seen using e.g. Brownian motion or capacity arguments. Thus, the boundary measurements for $(M, g)$ and $(\tilde{M}, \widetilde{g})$ coincide. This gives a counter example for the inverse electrostatic problem on Riemannian surfaces - even the topology of possibly non-compact Riemannian surfaces can not be determined using boundary measurements (see Fig. 1). The above example can be thought as a "hole" in a Riemann surface that does not change the boundary measurements. Roughly speaking, mapping the manifold $\widetilde{M}$ smoothly to the set $M \backslash \bar{B}_{M}\left(x_{0}, \rho\right)$, where $B_{M}\left(x_{0}, \rho\right)$ is a metric ball of $M$, and by putting an object in the obtained hole $\bar{B}_{M}\left(x_{0}, \rho\right)$, one could hide it from detection at the boundary. This observation was used in $[64,65]$, where "undetectability" results were introduced in three dimensions, using degenerations of Riemannian metrics, whose singular limits can be considered as coming directly from singular changes of variables. The degeneration of the metric (see Fig. 2) can be obtained by considering surfaces (or manifolds in the higher dimensional cases) with a thin "neck" that is pinched. At the limit the manifold contains a pocket about which the boundary measurements do not give any information. If the collapsing of the manifold is done in an appropriate way, we have, in the limit, a singular Riemannian manifold which is indistinguishable in boundary measurements from a flat surface. Then the conductivity which corresponds to this metric is also singular at the pinched points, cf. the first formula in (130). The electrostatic measurements on the boundary for this singular conductivity will be the same as for the original regular conductivity corresponding to the metric $g$. To give a precise, and concrete, realization of this idea, let $B(0, R) \subset \mathbb{R}^{3}$ denote the open ball with center 0 and radius $R$. We use in the sequel the set $N=B(0,2)$, the region at the boundary of which the electrostatic measurements will be made, decomposed into two parts, $N_{1}=B(0,2) \backslash \bar{B}(0,1)$ and 

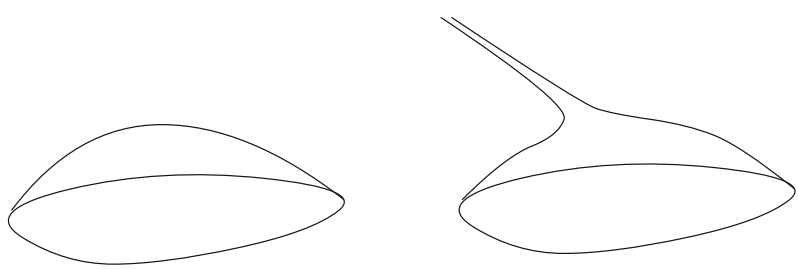

Fig. 1 Blowing up a metric at a point, after [125]. The electrostatic boundary measurements on the boundary of the surfaces, one compact and the other noncompact but complete, coincide

Fig. 2 A typical member of a family of manifolds developing a singularity as the width of the neck connecting the two parts goes to zero

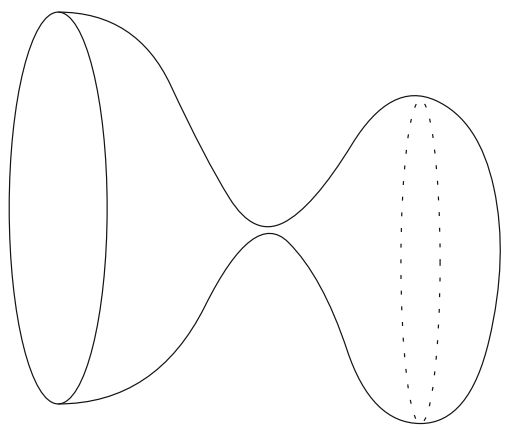

$N_{2}=B(0,1)$. We call the interface $\Sigma=\partial N_{2}$ between $N_{1}$ and $N_{2}$ the cloaking surface. We also use a "copy" of the ball $B(0,2)$, with the notation $M_{1}=B(0,2)$, another ball $M_{2}=B(0,1)$, and the disjoint union $M$ of $M_{1}$ and $M_{2}$. (We will see the reason for distinguishing between $N$ and $M$.) Let $g_{j k}=\delta_{j k}$ be the Euclidian metrics in $M_{1}$ and $M_{2}$ and let $\gamma=1$ be the corresponding isotropic homogeneous conductivity. We define a singular transformation

$$
F_{1}: M_{1} \backslash\{0\} \rightarrow N_{1}, \quad F_{1}(x)=\left(\frac{|x|}{2}+1\right) \frac{x}{|x|}, \quad 0<|x| \leq 2,
$$

(see Fig. 3).

We also consider a regular transformation (diffeomorphism) $F_{2}: M_{2} \rightarrow N_{2}$, which for simplicity we take to be the identity map $F_{2}=I d$. Considering the maps $F_{1}$ and $F_{2}$ together, $F=\left(F_{1}, F_{2}\right)$, we define a map $F: M \backslash\{0\}=\left(M_{1} \backslash\{0\}\right) \cup M_{2} \rightarrow N \backslash \Sigma$. The push-forward $\widetilde{g}=F_{*} g$ of the metric $g$ in $M$ by $F$ is the metric in $N$ given by

$$
\left(F_{*} g\right)_{j k}(y)=\left.\sum_{p, q=1}^{n} \frac{\partial F^{p}}{\partial x^{j}}(x) \frac{\partial F^{q}}{\partial x^{k}}(x) g_{p q}(x)\right|_{x=F^{-1}(y)} .
$$

This metric gives rise to a conductivity $\widetilde{\sigma}$ in $N$ which is singular in $N_{1}$,

$$
\widetilde{\sigma}= \begin{cases}|\widetilde{g}|^{1 / 2} \widetilde{g}^{j k} & \text { for } x \in N_{1} \\ \delta^{j k} & \text { for } x \in N_{2}\end{cases}
$$




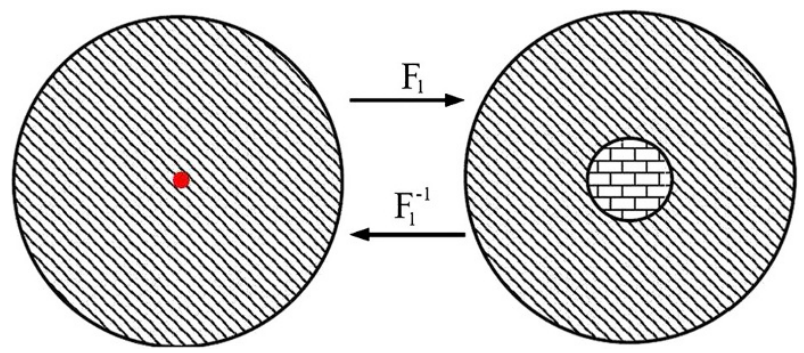

Fig. 3 Map $F_{1}: B(0,2) \backslash\{0\} \rightarrow B(0,2) \backslash \bar{B}(0,1)$

Thus, $F$ forms an invisibility construction that we call "blowing up a point". Denoting by $(r, \phi, \theta) \mapsto(r \sin \theta \cos \phi, r \sin \theta \sin \phi, r \cos \theta)$ the spherical coordinates, we have

$$
\tilde{\sigma}=\left(\begin{array}{lll}
2(r-1)^{2} \sin \theta & 0 & 0 \\
0 & 2 \sin \theta & 0 \\
0 & 0 & 2(\sin \theta)^{-1}
\end{array}\right), \quad 1<|x| \leq 2
$$

Note that the anisotropic conductivity $\widetilde{\sigma}$ is singular degenerate on $\Sigma$ in the sense that it is not bounded from below by any positive multiple of $I$. (See [112] for a similar calculation.) The Euclidian conductivity $\delta^{j k}$ in $N_{2}$ (130) could be replaced by any smooth conductivity bounded from below and above by positive constants. This would correspond to cloaking of a general object with non-homogeneous, anisotropic conductivity. Here, we use the Euclidian metric just for simplicity. Consider now the Cauchy data of all solutions in the Sobolev space $H^{1}(N)$ of the conductivity equation corresponding to $\widetilde{\sigma}$, that is,

$$
C_{1}(\widetilde{\sigma})=\left\{\left(\left.u\right|_{\partial N},\left.v \cdot p \widetilde{\sigma} \nabla u\right|_{\partial N}\right): u \in H^{1}(N), \nabla \cdot p \widetilde{\sigma} \nabla u=0\right\},
$$

where $v$ is the Euclidian unit normal vector of $\partial N$.

Theorem 3.1 ([65]) The Cauchy data of all $H^{1}$-solutions for the conductivities $\widetilde{\sigma}$ and $\gamma$ on $N$ coincide, that is, $C_{1}(\widetilde{\sigma})=C_{1}(\gamma)$.

This means that all boundary measurements for the homogeneous conductivity $\gamma=1$ and the degenerated conductivity $\tilde{\sigma}$ are the same. The result above was proven in $[64,65]$ for the case of dimension $n \geq 3$. The same basic construction works in the two dimensional case [112]. Figure 4 portrays an analytically obtained solution on a disc with conductivity $\widetilde{\sigma}$. As seen in the figure, no currents appear near the center of the disc, so that if the conductivity is changed near the center, the measurements on the boundary $\partial N$ do not change. The above invisibility result is valid for a more general class of singular cloaking transformations. A general class, sufficing at least for electrostatics, is given by the following result from [65]:

Theorem 3.2 Let $\Omega \subset \mathbb{R}^{n}, n \geq 3$, and $g=\left(g_{i j}\right)$ a smooth metric on $\Omega$ bounded from above and below by positive constants. Let $D \subset \subset \Omega$ be such that there is a $C^{\infty}$-diffeomorphism $F: \Omega \backslash\{y\} \rightarrow \Omega \backslash \bar{D}$ satisfying $\left.F\right|_{\partial \Omega}=I d$ and such that 


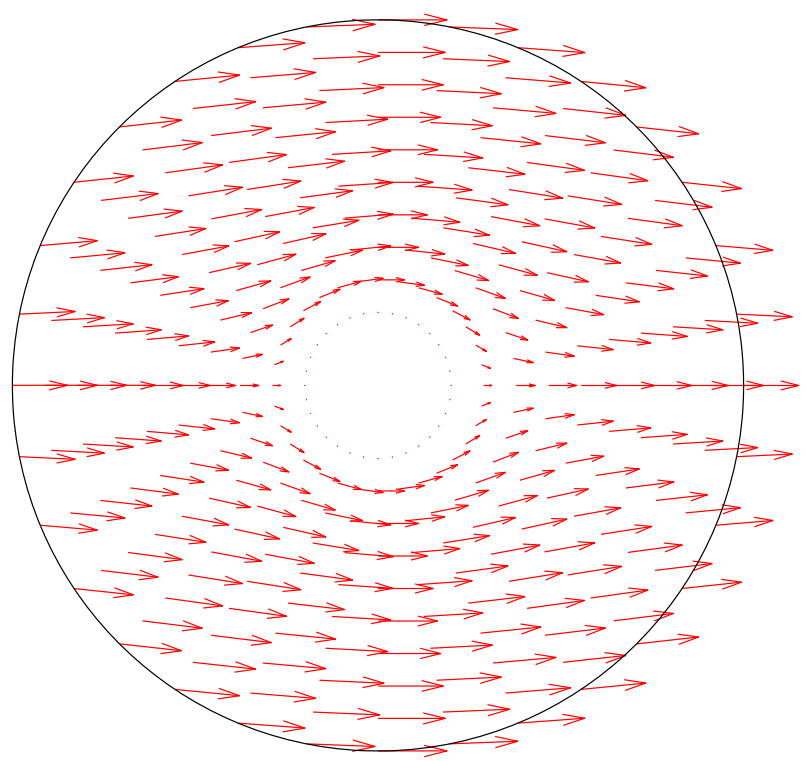

Fig. 4 Analytic solutions for the currents

$$
d F(x) \geq c_{0} I, \quad \operatorname{det}(d F(x)) \geq c_{1} \operatorname{dist}_{\mathbb{R}^{n}}(x, y)^{-1}
$$

where $d F$ is the Jacobian matrix in Euclidian coordinates on $\mathbb{R}^{n}$ and $c_{0}, c_{1}>0$. Let $\widehat{g}$ be a metric in $\Omega$ which coincides with $\widetilde{g}=F_{*} g$ in $\Omega \backslash \bar{D}$ and is an arbitrary regular positive definite metric in $D^{\text {int }}$. Finally, let $\sigma$ and $\widehat{\sigma}$ be the conductivities corresponding to $g$ and $\widehat{g}, c f$. (88). Then,

$$
C_{1}(\widehat{\sigma})=C_{1}(\sigma)
$$

The key to the proof of Theorem 3.2 is a removable singularities theorem that implies that solutions of the conductivity equation in $\Omega \backslash \bar{D}$ pull back by this singular transformation to solutions of the conductivity equation in the whole $\Omega$. Returning to the case $\Omega=N$ and the conductivity given by (130), similar types of results are valid also for a more general class of solutions. Consider an unbounded quadratic form, $A$ in $L^{2}\left(N,|\widetilde{g}|^{1 / 2} d x\right)$,

$$
A_{\tilde{\sigma}}[u, v]=\int_{N} \tilde{\sigma} \nabla u \cdot p \nabla v d x
$$

defined for $u, v \in \mathcal{D}\left(A_{\widetilde{\sigma}}\right)=C_{0}^{\infty}(N)$. Let $\bar{A}_{\widetilde{\sigma}}$ be the closure of this quadratic form and say that

$$
\nabla \cdot p \widetilde{\sigma} \nabla u=0 \text { in } N
$$


is satisfied in the finite energy sense if there is $u_{0} \in H^{1}(N)$ supported in $N_{1}$ such that $u-u_{0} \in \mathcal{D}\left(\bar{A}_{\tilde{\sigma}}\right)$ and

$$
\bar{A}_{\widetilde{\sigma}}\left[u-u_{0}, v\right]=-\int_{N} \widetilde{\sigma} \nabla u_{0} \cdot p \nabla v d x, \quad \text { for all } v \in \mathcal{D}\left(\bar{A}_{\widetilde{\sigma}}\right) .
$$

Then the Cauchy data set of the finite energy solutions, denoted by

$C_{f . e .}(\widetilde{\sigma})=\left\{\left(\left.u\right|_{\partial N},\left.v \cdot p \widetilde{\sigma} \nabla u\right|_{\partial N}\right) \mid u\right.$ is a finite energy solution of $\left.\nabla \cdot p \widetilde{\sigma} \nabla u=0\right\}$

coincides with the Cauchy data $C_{\text {f.e. }}(\gamma)$ corresponding to the homogeneous conductivity $\gamma=1$, that is,

$$
C_{f . e .}(\widetilde{\sigma})=C_{f . e .}(\gamma)
$$

Kohn, Shen, Vogelius and Weinstein [112] in an interesting article have considered the case when instead of blowing up a point one stretches a small ball into the cloaked region. In this case the conductivity is non-singular and one gets "almost" invisibility with a precise estimate in terms of the radius of the small ball.

\subsection{Quantum shielding}

In [63], using CGO solutions, uniqueness was proven for the Calderón problem for Schrödinger operators having a more singular class of potentials, namely potentials conormal to submanifolds of $\mathbb{R}^{n}, n \geq 3$. However, for more singular potentials, there are counterexamples to uniqueness. It was constructed in [63] a class of potentials that shield any information about the values of a potential on a region $D$ contained in a domain $\Omega$ from measurements of solutions at $\partial \Omega$. In other words, the boundary information obtained outside the shielded region is independent of $\left.q\right|_{D}$. On $\Omega \backslash D$, these potentials behave like $q(x) \sim-C d(x, \partial D)^{-2-\epsilon}$ where $d$ denotes the distance to $\partial D$ and $C$ is a positive constant. In $D$, Schrödinger's cat could live forever. From the point of view of quantum mechanics, $q$ represents a potential barrier so steep that no tunneling can occur. From the point of view of optics and acoustics, no sound waves or electromagnetic waves will penetrate, or emanate from, $D$. However, this construction should be thought of as shielding, not cloaking, since the potential barrier that shields $\left.q\right|_{D}$ from boundary observation is itself detectable .

Open Access This article is distributed under the terms of the Creative Commons Attribution License which permits any use, distribution, and reproduction in any medium, provided the original author(s) and the source are credited. 


\section{References}

1. Ablowitz, M., Yaacov, D.B., Fokas, A.: On the inverse scattering transform for the KadomtsevPetviashvili equation. Stud. Appl. Math. 69, 135-143 (1983)

2. Ahlfors, L.: Quasiconformal Mappings. Van Nostrand, Princeton (1966)

3. Albin, P., Guillarmou, C., Tzou, L., Uhlmann, G.: Inverse boundary problems for systems in two dimensions. Annales Institut Henri Poincaré 14, 1151-1571 (2013)

4. Alessandrini, G.: Stable determination of conductivity by boundary measurements. App. Anal. 27, 153-172 (1988)

5. Alessandrini, G.: Singular solutions of elliptic equations and the determination of conductivity by boundary measurements. J. Differ. Equ. 84, 252-272 (1990)

6. Alessandrini, G.: Open issues of stability for the inverse conductivity problem. J. Inverse Ill-Posed Probl. 15, 451-460 (2007)

7. Alessandrini, G., Vessella, S.: Lipschitz stability for the inverse conductivity problem. Adv. Appl. Math. 35, 207-241 (2005)

8. Alexandrova, I.: Structure of the semi-classical amplitude for general scattering relations. Comm. PDE 30, 1505-1535 (2005)

9. Ammari, H., Uhlmann, G.: Reconstruction of the potential from partial Cauchy data for the Schrödinger equation. Indiana Univ. Math. J. 53, 169-183 (2004)

10. Anikonov, YuE: Some Methods for the Study of Multidimensional Inverse Problems. Nauka. Sibirsk Otdel, Novosibirsk (1978)

11. Astala, K., Päivärinta, L.: Calderón's inverse conductivity problem in the plane. Ann. Math. 163, 265-299 (2006)

12. Astala, K., Lassas, M., Päiväirinta, L.: Calderón's inverse problem for anisotropic conductivity in the plane. Comm. Partial Differ. Equ. 30, 207-224 (2005)

13. Bal, G.: Hybrid inverse problems and internal functionals. In: Uhlmann, G. (ed.) Chapter in Inside Out II, MSRI Publications, vol. 60, pp. 271-323. Cambridge University Press, Cambridge (2012)

14. Bal, G., Langmore, I., Monard, F.: Inverse transport with isotropic sources and angularly averaged measurements. Inverse Probl. Imaging 2, 23-42 (2008)

15. Bal, G., Ren, K., Uhlmann, G., Zhou, T.: Quantitative thermo-acoustics and related problems. Inverse Probl. 27, 055007 (2011)

16. Bal, G., Uhlmann, G.: Inverse diffusion theory of photoacoustics. Inverse Probl. 26, 085010 (2010)

17. Bal, G., Uhlmann, G.: Reconstructions for some coupled-physics inverse problems. Appl. Math. Lett. 25, 1030-1033 (2012)

18. Bal, G., Uhlmann, G.: Reconstructions of coefficients in scalar second-order elliptic equations from knowledge of their solutions. Comm. Pure Appl. Math. 66, 1629-1652

19. Barber, D., Brown, B.: Applied potential tomography. J. Phys. E 17, 723-733 (1984)

20. Barceló, T., Faraco, D., Ruiz, A.: Stability of Calderón's inverse problem in the plane. Journal des Mathématiques Pures et Appliquées 88, 522-556 (2007)

21. Beals, R., Coifman, R.: Transformation spectrales et equation d'evolution non lineares. Seminaire Goulaouic-Meyer-Schwarz, exp. 21 (1981-1982)

22. Beals, R., Coifman, R.: Multidimensional inverse scattering and nonlinear PDE. Proc. Symp. Pure Math., vol. 43, pp. 45-70. American Mathematical Society, Providence (1985).

23. Belishev, M.I.: The Calderón problem for two-dimensional manifolds by the BC-method. SIAM J. Math. Anal. 35, 172-182 (2003)

24. Belishev, M., Kurylev, Y.: To the reconstruction of a Riemannian manifold via its spectral data (BCmethod). Comm. Partial Differ. Equ. 17, 767-804 (1992)

25. Bernstein, I.N., Gerver, M.L.: Conditions on distinguishability of metrics by hodographs. In: Methods and Algorithms of Interpretation of Seismological Information, Computerized Seismology, vol. 13, pp. 50-73. Nauka, Moscow (1980) (in Russian)

26. Besson, G., Courtois, G., Gallot, S.: Entropies et rigidités des espaces localement symétriques de courbure strictment négative. Geom. Funct. Anal. 5, 731-799 (1995)

27. Beylkin, G.: Stability and uniqueness of the solution of the inverse kinematic problem in the multidimensional case. J. Soviet Math. 21, 251-254 (1983)

28. Blasten, E.: Stability and uniqueness for the inverse problem of the Schrödinger equation with potentials in $W^{p, \epsilon}$. http://arxiv.org/abs/1106.0632arXiv:1106.0632

29. Borcea, L.: Electrical impedance tomography. Inverse Probl. 18, R99-R136 (2002) 
30. Borcea, L., Druskin, V., Guevara Vasquez, F., Mamonov, A.V.: Resistor network approaches to electrical impedance tomography. In: Uhlmann, G. (ed.) Inside Out II. MSRI Publications, vol. 60, pp. 55-118 (2012).

31. Brown, R.: Recovering the conductivity at the boundary from the Dirichlet to Neumann map: a pointwise result. J. Inverse Ill-Posed Probl. 9, 567-574 (2001)

32. Brown, R., Torres, R.: Uniqueness in the inverse conductivity problem for conductivities with $3 / 2$ derivatives in $L^{p}, p>2 n$. J. Fourier Analysis Appl. 9, 1049-1056 (2003)

33. Brown, R., Uhlmann, G.: Uniqueness in the inverse conductivity problem with less regular conductivities in two dimensions. Comm. PDE 22, 1009-10027 (1997)

34. Bukhgeim, A.: Recovering the potential from Cauchy data in two dimensions. J. Inverse Ill-Posed Probl. 16, 19-34 (2008)

35. Bukhgeim, A., Uhlmann, G.: Recovering a potential from partial Cauchy data. Comm. PDE 27, 653-668 (2002)

36. Burago, D., Ivanov, S.: Boundary rigidity and filling volume minimality for metrics close to a Euclidean metric. Annals of Math. 171, 1183-1211 (2010)

37. Calderón, A.P.: On an inverse boundary value problem. In: Seminar on Numerical Analysis and its Applications to Continuum Physics (Rio de Janeiro, 1980), pp. 65-73. Soc. Brasil. Mat., Rio de Janeiro (1980)

38. Calderón, A.P.: Reminiscencias de mi vida matemática, Discurso de investidura de "Doctor Honoris Causa", pp. 117-125. Publicaciones UAM, Universidad Autónoma de Madrid (1997)

39. Calderón, A.P.: Boundary value problems for elliptic equations. Outlines of the joint Soviet-American symposium on partial differential equations, pp. 303-304, Novisibirsk (1963)

40. Caro, P., Ola, P., Salo, M.: Inverse boundary value problem for Maxwell equations with local data. Comm. PDE 34, 1425-1464 (2009)

41. Chanillo, S.: A problem in electrical prospection and a $n$-dimensional Borg-Levinson theorem. Proc. AMS 108, 761-767 (1990)

42. Chen, J., Yang, Y.: Quantitative photo-acoustic tomography with partial data. Inverse Probl. 28, 115014 (2012)

43. Cheney, M., Isaacson, D., Newell, J.C.: Electrical impedance tomography. SIAM Rev. 41, 85-101 (1999)

44. Creager, K.C.: Anisotropy of the inner core from differential travel times of the phases PKP and PKIPK. Nature 356, 309-314 (1992)

45. Croke, C.: Rigidity and the distance between boundary points. J. Differ. Geom. 33, 445-464 (1991)

46. Croke, C.: Rigidity for surfaces of non-positive curvature. Comment. Math. Helv. 65, 150-169 (1990)

47. Croke, C., Dairbekov, D., Sharafutdinov, V.: Local boundary rigidity of a compact Riemannian manifold with curvature bounded above. Trans. Amer. Math. Soc. 352(9), 3937-3956 (2000)

48. Croke, C., Kleiner, B.: Conjugacy and rigidity for manifolds with a parallel vector field. J. Differ. Geom. 39, 659-680 (1994)

49. Dos Santos Ferreira, D., Kenig, C.E., Sjöstrand, J., Uhlmann, G.: On the linearized local Calderón problem. G. Math. Res. Lett. 16, 955-970 (2009)

50. Dairbekov, N., Uhlmann, G.: Reconstructing the metric and magnetic field from the scattering relation. Inverse Probl. Imaging 4, 397-409 (2010)

51. Ferreira, Dos Santos: D., Kenig, C.E., Sjöstrand, J., Uhlmann, G.: Determining a magnetic Schrödinger operator from partial Cauchy data. Comm. Math. Phys. 271, 467-488 (2007)

52. Dos Santos Ferreira, D., Kenig, C.E., Salo, M., Uhlmann, G.: Limiting Carleman weights and anisotropic inverse problems. Inventiones Math. 178, 119-171 (2009)

53. Duistermaat, J.J., Hörmander, L.: Fourier integral operators II. Acta Math. 128, 183-269 (1972)

54. Eisenhart, L.: Riemannian geometry, 2nd printing. Princeton University Press, Princeton (1949)

55. Eskin, G., Ralston, J.: On the inverse boundary value problem for linear isotropic elasticity. Inverse Probl. 18, 907-921 (2002)

56. Faddeev D.: Growing solutions of the Schrödinger equation. Dokl. Akad. Nauk SSSR 165, 514-517 (1965) (translation in, Sov. Phys. Dokl. 10, 1033)

57. Francini, E.: Recovering a complex coefficient in a planar domain from the Dirichlet-to-Neumann map. Inverse Probl. 16, 107-119 (2000)

58. Fridman, B., Kuchment, P., Ma, D., Papanicolaou, V.G.: Solution of the linearized inverse conductivity problem in a half space via integral geometry. Voronezh Winter Mathematical Schools, pp. 85-95. 
Amer. Math. Soc. Transl. Ser. 2, 184, pp. 85-95. American Mathematical Society, Providence, RI (1998)

59. Gilbarg, D., Trudinger, N.: Elliptic Partial Differential Equations. Interscience Publishers, New York (1964)

60. Gerver, M.L., Nadirashvili, N.S.: An isometricity conditions for Riemannian metrics in a disk. Soviet Math. Dokl. 29, 199-203 (1984)

61. Greenleaf, A., Kurylev, Y., Lassas, M., Uhlmann, G.: Cloaking devices, electromagnetic wormholes, and transformation optics. SIAM Rev. 51, 3-33 (2009)

62. Greenleaf, A., Kurylev, Y., Lassas, M., Uhlmann, G.: Invisibility and inverse problems. Bull. Amer. Math. Soc. (N.S.) 46, 55-97 (2009)

63. Greenleaf, A., Lassas, M., Uhlmann, G.: The Calderón problem for conormal potentials, I: Global uniqueness and reconstruction. Comm. Pure Appl. Math 56, 328-352 (2003)

64. Greenleaf, A., Lassas, M., Uhlmann, G.: Anisotropic conductivities that cannot be detected in EIT. Physiolog. Meas. (special issue on Impedance Tomography) 24, 413-420 (2003)

65. Greenleaf, A., Lassas, M., Uhlmann, G.: On nonuniqueness for Calderón's inverse problem. Math. Res. Lett. 10, 685-693 (2003)

66. Greenleaf, A., Uhlmann, G.: Local uniqueness for the Dirichlet-to-Neumann map via the two-plane transform. Duke Math. J. 108, 599-617 (2001)

67. Gromov, M.: Filling Riemannian manifolds. J. Differ. Geom. 18(1), 1-148 (1983)

68. Inverse problems for Einstein manifolds: Guillarmou, C., Sá Barreto, A. Inverse Probl. Imag. 3, 1-15 (2009)

69. Guillarmou, C., Tzou, L.: Calderón inverse problem on Riemann surfaces. In: Proceedings of CMA, vol. 44, pp. 129-142. Volume for the AMSI/ANU workshop on Spectral Theory and Harmonic, Analysis (2009)

70. Guillarmou, C., Tzou, L.: Calderón inverse problem with partial data on Riemann surfaces. Duke Math. J. 158, 83-120 (2011)

71. Guillarmou, C., Tzou, L.: Identification of a connection from Cauchy data space on a Riemann surface with boundary. Geom. Funct. Anal. (GAFA) 21, 393-418 (2011)

72. Guillemin, V.: Sojourn times and asymptotic properties of the scattering matrix. In: Proceedings of the Oji Seminar on Algebraic Analysis and the RIMS Symposium on Algebraic Analysis (Kyoto Univ., Kyoto, 1976). Publ. Res. Inst. Math. Sci., vol. 12, supplement, pp. 69-88 (1976/77).

73. Hähner, P.: A periodic Faddeev-type solution operator. J. Differ. Equ. 128, 300-308 (1996)

74. Hanke, M., Brühl, M.: Recent progress in electrical impedance tomography. Special Sect. Imaging Inverse Probl. 19, S65-S90 (2003)

75. Haberman, B., Tataru, D.: Uniqueness in Calderón's problem with Lipschitz conductivities. Duke Math. J. 162, 497-516 (2013)

76. Heck, H., Wang, J.-N.: Stability estimates for the inverse boundary value problem by partial Cauchy data. Inverse Probl. 22, 1787-1796 (2006)

77. Henkin, G., Michel, V.: Inverse conductivity problem on Riemann surfaces. J. Geom. Anal. 18, $1033-1052$ (2008)

78. Herglotz, G.: Uber die elastizitaet derErde bei beruecksichtigung ihrer variablen dichte. Zeitschr. fur Math. Phys. 52, 275-299 (1905)

79. Holder, D.: Electrical Impedance Tomography. Institute of Physics Publishing, Bristol and Philadelphia (2005)

80. Holder, D., Isaacson, D., Müller, J., Siltanen, S. (eds.) Physiol. Meas. 25(1) (2003)

81. Hörmander, L.: The Analysis of Linear Partial Differential Operators, vol. I. Springer, Berlin (1983)

82. Ide, T., Isozaki, H., Nakata, S., Siltanen, S., Uhlmann, G.: Probing for electrical inclusions with complex spherical waves. Comm. Pure Appl. Math. 60, 1415-1442 (2007)

83. Ikehata, M.: The enclosure method and its applications. In: Analytic extension formulas and their applications, Chapter 7. (Fukuoka, 1999/Kyoto, 2000). Int. Soc. Anal. Appl. Comput., vol. 9, pp. 87-103. Kluwer Academic Publishers, Boston (2001)

84. Ikehata, M.: How to draw a picture of an unknown inclusion from boundary measurements. Two mathematical inversion algorithms. J. Inverse Ill-Posed Probl. 7, 255-271 (1999)

85. Ikehata, M., Siltanen, S.: Numerical method for finding the convex hull of an inclusion in conductivity from boundary measurements. Inverse Probl. 16, 273-296 (2000)

86. Imanuvilov, O., Yamamoto, M.: Inverse boundary value for Schrödinger equation in two dimensions, arXiv. http://arxiv.org/abs/1211.1419v1arXiv:1211.1419v1 
87. Imanuvilov, O., Uhlmann, G., Yamamoto, M.: The Calderón problem with partial data in two dimensions. J. AMS 23, 655-691 (2010)

88. Imanuvilov, O., Uhlmann, G., Yamamoto, M.: On determination of second order operators from partial Cauchy data. Proc. Natl. Acad. Sci. 108, 467-472 (2011)

89. Imanuvilov, O., Uhlmann, G., Yamamoto, M.: Partial data for general second order elliptic operators in two dimensions. Publ. Res. Inst. Math. Sci. 48, 971-1055 (2012)

90. Imanuvilov, O., Uhlmann, G., Yamamoto, M.: Inverse boundary problem with Cauchy data on disjoint sets. Inverse Probl. 27, 085007 (2011)

91. Imanuvilov, O., Uhlmann, G., Yamamoto, M.: On reconstruction of Lamé coefficients from partial Cauchy data in three dimensions. Inverse Probl. 28, 125002 (2012)

92. Isaacson, D., Isaacson, E.: Comment on Calderón's paper: "On an inverse boundary value problem". Math. Comput. 52, 553-559 (1989)

93. Isaacson, D., Müller, J.L., Newell, J.C., Siltanen, S.: Reconstructions of chest phantoms by the d-bar method for electrical impedance tomography. IEEE Trans. Med. Imaging 23, 821-828 (2004)

94. Isaacson, D., Newell, J.C., Goble, J.C., Cheney, M.: Thoracic impedance images during ventilation. Ann. Conf. IEEE Eng. Med. Biol. Soc. 12, 106-107 (1990)

95. Isakov, V.: On uniqueness in the inverse conductivity problem with local data. Inverse Probl. Imaging 1, 95-105 (2007)

96. Isakov, V.: On uniqueness in inverse problems for semilinear parabolic equations. Arch. Rat. Mech. Anal. 124, 1-12 (1993)

97. Isakov, V.: Completeness of products of solutions and some inverse problems for PDE. J. Differ. Equ. 92, 305-317 (1991)

98. Isakov, V., Nachman, A.: Global uniqueness for a two-dimensional semilinear elliptic inverse problem. Trans. AMS 347, 3375-3390 (1995)

99. Isakov, V., Sylvester, J.: Global uniqueness for a semilinear elliptic inverse problem. Comm. Pure Appl. Math. 47, 1403-1410 (1994)

100. Isozaki, H.: Inverse spectral problems on hyperbolic manifolds and their applications to inverse boundary value problems in Euclidean space. Amer. J. Math. 126, 1261-1313 (2004)

101. Isozaki, H., Uhlmann, G.: Hyperbolic geometric and the local Dirichlet-to-Neumann map. Adv. Math. 188, 294-314 (2004)

102. Jordana, J., Gasulla, J.M., Paola's-Areny, R.: Electrical resistance tomography to detect leaks from buried pipes. Meas. Sci. Technol. 12, 1061-1068 (2001)

103. Jossinet, J.: The impedivity of freshly excised human breast tissue. Physiol. Meas. 19, 61-75 (1998)

104. Kang, H., Uhlmann, G.: Inverse problems for the Pauli Hamiltonian in two dimensions. J. Fourier Anal. Appl. 10, 201-215 (2004)

105. Kashiwara, M.: On the structure of hyperfunctions. Sagaku no Ayumi 15, 19-72 (1970). (in Japanese)

106. Kenig, C., Salo, M., Uhlmann, G.: Inverse Problems for the Anisotropic Maxwell's Equations. Duke Math. J. 157, 369-419 (2011)

107. Kenig, C., Sjöstrand, J., Uhlmann, G.: The Calderón problem with partial data. Ann. Math. 165, 567-591 (2007)

108. Knudsen, K.: The Calderón problem with partial data for less smooth conductivities. Comm. Partial Differ. Equ. 31, 57-71 (2006)

109. Knudsen, K., Salo, M.: Determining nonsmooth first order terms from partial boundary measurements. Inverse Probl. Imaging 1, 349-369 (2007)

110. Kocyigit, I.: Acoustic-electric tomography and CGO solutions with internal data. Inverse Probl. 28, 125004 (2012)

111. Kolehmainen, V., Lassas, M., Ola, P.: Inverse conductivity problem with an imperfectly known boundary. SIAM J. Appl. Math. 66, 365-383 (2005)

112. Kohn, R., Shen, H., Vogelius, M., Weinstein, M.: Cloaking via change of variables in electrical impedance tomography. Inverse Probl. 24, 015016 (2008)

113. Kohn, R., Vogelius, M.: Identification of an unknown conductivity by means of measurements at the boundary. Inverse Probl. SIAM-AMS Proc. 14 (1984)

114. Kohn, R., Vogelius, M.: Determining conductivity by boundary measurements. Comm. Pure Appl. Math. 37, 289-298 (1984)

115. Kohn, R., Vogelius, M.: Determining conductivity by boundary measurements II. Interior results. Comm. Pure Appl. Math. 38, 643-667 (1985) 
116. Krupchyk, K., Lassas, M., Uhlmann, G.: Inverse problems for differential forms on Riemannian manifolds with boundary". Comm. PDE. 36, 1475-1509 (2011)

117. Krupchyk, K., Lassas, M., Uhlmann, G.: Inverse problems with partial data for the magnetic Schrödinger operator in an infinite slab and on a bounded domain Comm. Math. Phys. 312, 87-126 (2012)

118. Krupchyk, K., Lassas, M., Uhlmann, G.: Inverse boundary value problems for the polyharmonic operator. J. Funct. Anal. 262, 1781-1801 (2012)

119. Krupchyk, K., Lassas, M., Uhlmann, G: Determining a first order perturbation of the biharmonic operator by partial boundary measurements. Trans. AMS 366, 95-112 (2014)

120. Krupchyk, K., Uhlmann, G.: Determining a magnetic Schrödinger operator with a bounded magnetic potential from boundary measurements. Comm. Math. Phys. (2014, to appear)

121. Lee, J., Uhlmann, G.: Determining anisotropic real-analytic conductivities by boundary measurements. Comm. Pure Appl. Math. 42, 1097-1112 (1989)

122. Li, X., Uhlmann, G.: Inverse problems on a slab. Inverse Probl. Imaging 4, 449-462 (2010)

123. Lassas, M., Sharafutdinov, V., Uhlmann, G.: Semiglobal boundary rigidity for Riemannian metrics. Math. Annalen 325, 767-793 (2003)

124. Lassas, M., Uhlmann, G.: Determining a Riemannian manifold from boundary measurements. Ann. Sci. École Norm. Sup. 34, 771-787 (2001)

125. Lassas, M., Taylor, M., Uhlmann, G.: The Dirichlet-to-Neumann map for complete Riemannian manifolds with boundary. Comm. Geom. Anal. 11, 207-222 (2003)

126. Leonhardt, U.: Optical Conformal Mapping. Science 312, 1777-1780 (2006)

127. Mandache, N.: Exponential instability in an inverse problem for the Schrödinger equation. Inverse Probl. 17, 1435-1444 (2001)

128. Melrose, R.B.: Geometric Scattering Theory. Cambridge University Press, Cambridge (1995)

129. Michel, R.: Sur la rigidité imposée par la longueur des géodésiques. Invent. Math. 65, 71-83 (1981)

130. Michel, R.: Restriction de la distance géodésique a un arc et rigidité. Bull. Soc. Math. France 122, 435-442 (1994)

131. Mukhometov, R.G.: The reconstruction problem of a two-dimensional Riemannian metric, and integral geometry (Russian). Dokl. Akad. Nauk SSSR 232(1), 32-35 (1977)

132. Mukhometov, R.G.: On one problem of reconstruction of Riemannian metric (Russian). Siberian Math. J. 22(3), 119-135 (1981)

133. Mukhometov, R.G., Romanov, V.G.: On the problem of finding an isotropic Riemannian metric in an n-dimensional space (Russian). Dokl. Akad. Nauk SSSR 243(1), 41-44 (1978)

134. Nachman, A.: Global uniqueness for a two-dimensional inverse boundary value problem. Ann. Math. 143, 71-96 (1996)

135. Nachman, A.: Reconstructions from boundary measurements. Ann. Math. 128, 531-576 (1988)

136. Nachman, A., Ablowitz, N.: A multidimensional inverse scattering method. Stud. Appl. Math. 71, 243-250 (1984)

137. Nachman, A., Street, B.: Reconstruction in the Calderón problem with partial data. Comm. PDE 35, 375-390 (2010)

138. Nagayasu, S., Uhlmann, G., Wang, J.-N.: Depth dependent stability estimate in electrical impedance tomography. Inverse Probl. 25, 075001 (2009)

139. Nagayasu, S., Uhlmann, G., Wang, J.-N.: Reconstruction of penetrable obstacles in acoustics. SIAM J. Math. Anal. 43, 189-211 (2011)

140. Nagayasu, S., Uhlmann, G., Wang, J.-N.: Increasing stability for the acoustic equation. Inverse Probl. 29, 229-247 (2013)

141. Nakamura, G., Tanuma, K.: Local determination of conductivity at the boundary from the Dirichletto-Neumann map. Inverse Probl. 17, 405-419 (2001)

142. Nakamura, G., Uhlmann, G.: Global uniqueness for an inverse boundary value problem arising in elasticity, Invent. Math., 118, : 457-474. Erratum. Invent. Math. 152(2003), 205-207 (1994)

143. Nakamura, G., Uhlmann, G.: Inverse problems at the boundary for an elastic medium. SIAM J. Math. Anal. 26, 263-279 (1995)

144. Nakamura, G., Sun, Z., Uhlmann, G.: Global identifiability for an inverse problem for the Schrödinger equation in a magnetic field. Math. Annalen 303, 377-388 (1995)

145. Novikov, R.G.: Multidimensional inverse spectral problems for the equation $-\Delta \psi+(v(x)$ $-E u(x)) \psi=0$, Funktsionalny Analizi Ego Prilozheniya 22, 11-12 (1988). Translation in Functional Analysis and its Applications 22, 263-272 (1988) 
146. Novikov, R.G., Henkin, G.M.: The $\bar{\partial}$-equation in the multidimensional inverse scattering problem. Russ. Math. Surv. 42, 109-180 (1987)

147. Ola, P., Päivärinta, L., Somersalo, E.: An inverse boundary value problem in electrodynamics. Duke Math. J. 70, 617-653 (1993)

148. Ola, P., Somersalo, E.: Electromagnetic inverse problems and generalized Sommerfeld potentials. SIAM J. Appl. Math. 56, 1129-1145 (1996)

149. Otal, J.P.: Sur les longuer des géodésiques d'une métrique a courbure négative dans le disque. Comment. Math. Helv. 65, 334-347 (1990)

150. Paternain, G., Salo, M., Uhlmann, G.: The attenuated ray transform for connections and Higgs fields. Geom. Funct. Anal. (GAFA) 22, 1460-1489 (2012)

151. Paternain, G., Salo, M., Uhlmann, G.: Tensor tomography on surfaces. Inventiones Math. 193, 2051820530 (2013)

152. Päivärinta, L., Panchenko, A., Uhlmann, G.: Complex geometrical optics for Lipschitz conductivities. Revista Matematica Iberoamericana 19, 57-72 (2003)

153. Pendry, J.B., Schurig, D., Smith, D.R.: Controlling electromagnetic fields. Science 312, 1780-1782 (2006)

154. Pestov, L., Sharafutdinov, V.A.: Integral geometry of tensor fields on a manifold of negative curvature. Siberian Math. J. 29, 427-441 (1988)

155. Pestov, L., Uhlmann, G.: Two dimensional simple Riemannian manifolds with boundary are boundary distance rigid. Ann. Math. 161, 1089-1106 (2005)

156. Pestov, L., Uhlmann, G.: The boundary distance function and the Dirichlet-to-Neumann map. Math. Res. Lett. 11, 285-298 (2004)

157. Pestov, P., Uhlmann, G.: Characterization of the range and inversion formulas for the geodesic X-ray transform. Int. Math. Res. Notices 80, 4331-4347 (2004)

158. Petersen, P.: Riemannian Geometry. Springer, Berlin (1998)

159. Ramm, A.G.: Recovery of the potential from fixed energy scattering data. Inverse Probl. 4, 877-886 (1988)

160. Rondi, L.: A remark on a paper by G. Alessandrini and S. Vessella: "Lipschitz stability for the inverse conductivity problem" [Adv. Appl. Math. 35, 207-241 (2005)]. Adv. Appl. Math. 36, 67-69 (2006).

161. Romanov, V.G.: Inverse Problems of Mathematical Physics. VNU Science Press, Utrech, the Netherlands (1987)

162. Salo, M.: Semiclassical pseudodifferential calculus and the reconstruction of a magnetic field. Comm. PDE 31, 1639-1666 (2006)

163. Salo, M., Inverse problems for nonsmooth first order perturbations of the Laplacian. Ann. Acad. Sci. Fenn. Math. Diss. 139 (2004)

164. Salo, M., Tzou, L.: Inverse problems with partial data for a Dirac system: a Carleman estimate approach. Adv. Math. 225, 487-513 (2010)

165. Salo, M., Wang, J.-N.: Complex spherical waves and inverse problems in unbounded domains. Inverse Probl. 22, 2299-2309 (2006)

166. Santosa, F., Vogelius, M.: A backprojection algorithm for electrical impedance imaging. SIAM J. Appl. Math. 50, 216-243 (1990)

167. Sharafutdinov, V.: Integral geometry of tensor fields. In: Inverse and Ill-Posed Problems Series, VSP, Utrecht (1994)

168. Sharafutdinov, V.A.: Variations of Dirichlet-to-Neumann map and deformation boundary rigidity of simple 2-manifolds. J. Geom. Anal. 17, 147-187 (2007)

169. Sharafutdinov, V., Skokan, M., Uhlmann, G.: Regularity of ghosts in tensor tomography. J. Geom. Anal. 15, 517-560 (2005)

170. Sharafutdinov, V., Uhlmann, G.: On deformation boundary rigidity and spectral rigidity for Riemannian surfaces with no focal points. J. Differ. Geom. 56, 93-110 (2001)

171. Schurig, D., Mock, J., Justice, B., Cummer, S., Pendry, J., Starr, A., Smith, D.: Metamaterial electromagnetic cloak at microwave frequencies. Science 314, 977-980 (2006)

172. Siltanen, S., Müller, J.L., Isaacson, D.: A direct reconstruction algorithm for electrical impedance tomography. IEEE Trans. Med. Imaging 21, 555-559 (2002)

173. Sjöstrand, J., Singularités analytiques microlocales, Astérisque (1985)

174. Sjöstrand, J.: Remark on extensions of the Watermelon theorem. Math. Res. Lett. 1, 309-317 (1994)

175. Somersalo, E., Isaacson, D., Cheney, M.: A linearized inverse boundary value problem for Maxwell's equations. J. Comput. Appl. Math. 42, 123-136 (1992) 
176. Stefanov, P., Uhlmann, G.L.: Multi-wave methods via ultrasound. In: Uhlmann, G. (ed.) Inverse Problems and Applications, Inside Out II, MSRI Publications, vol. 60, pp. 271-323. Cambridge University Press, Cambridge (2012)

177. Stefanov, P., Uhlmann, G.: Recent progress on the boundary rigidity problem. Electr. Res. Announc. Amer. Math. Soc. 11, 64-70 (2005)

178. Stefanov, P., Uhlmann, G.: Rigidity for metrics with the same lengths of geodesics. Math. Res. Lett. 5, 83-96 (1998)

179. Stefanov, P., Uhlmann, G.: Stability estimates for the X-ray transform of tensor fields and boundary rigidity. Duke Math. J. 123, 445-467 (2004)

180. Stefanov, P., Uhlmann, G.: Stable determination of generic simple metrics from the hyperbolic Dirichlet-to-Neumann map. Int. Math. Res. Notices 17, 1047-1061 (2005)

181. Stefanov, P., Uhlmann, G.: Boundary rigidity and stability for generic simple metrics. J. Amer. Math. Soc. 18, 975-1003 (2005)

182. Stefanov, P., Uhlmann, G.: Integral geometry of tensor fields on a class of non-simple Riemannian manifolds. Am. J. Math. 130, 239-268 (2008)

183. Stefanov, P., Uhlmann, G.: Local lens rigidity with incomplete data for a class of non-simple Riemannian manifolds. J. Differ. Geom. 82, 383-409 (2009)

184. Sun, Z.: On a quasilinear boundary value problem. Math. Z. 221, 293-305 (1996)

185. Sun, Z.: Conjectures in inverse boundary value problems for quasilinear elliptic equations. Cubo 7 , 65-73 (2005)

186. Sun, Z., Uhlmann, G.: Anisotropic inverse problems in two dimensions. Inverse Probl. 19, 1001-1010 (2003)

187. Sun, Z., Uhlmann, G.: Generic uniqueness for an inverse boundary value problem. Duke Math. J. 62, 131-155 (1991)

188. Sun, Z., Uhlmann, G.: Inverse problems in quasilinear anisotropic media. Amer. J. Math. 119, 771-797 (1997)

189. Sylvester, J.: An anisotropic inverse boundary value problem. Comm. Pure Appl. Math. 43, 201-232 (1990)

190. Sylvester, J., Uhlmann, G.: A global uniqueness theorem for an inverse boundary value problem. Ann. Math. 125, 153-169 (1987)

191. Sylvester, J., Uhlmann, G.: A uniqueness theorem for an inverse boundary value problem in electrical prospection. Comm. Pure Appl. Math. 39, 92-112 (1986)

192. Sylvester, J., Uhlmann, G.: Inverse boundary value problems at the boundary - continuous dependence. Comm. Pure Appl. Math. 41, 197-221 (1988)

193. Sylvester, J., Uhlmann, G.: Inverse problems in anisotropic media. Contemp. Math. 122, 105-117 (1991)

194. Tataru, D.: Unique continuation for solutions to PDE's; between Hörmander's theorem and Holmgren's theorem. Comm. PDE 20, 855-884 (1995)

195. Treves, F.: Introduction to pseudodifferential and fourier integral operators, vol. 1. In: Pseudodifferential Operators. The University Series in Mathematics, Plenum Press, New York (1980)

196. Tsai, T.Y.: The Schrödinger equation in the plane. Inverse Probl. 9, 763-787 (1993)

197. Tolmasky, C.: Exponentially growing solutions for nonsmooth first-order perturbations of the Laplacian. SIAM J. Math. Anal. 29, 116-133 (1998)

198. Tzou, L.: Stability estimates for coefficients of magnetic Schrödinger equation from full and partial measurements. Comm. PDE 33, 161-184 (2008)

199. Uhlmann, G., Inverse boundary value problems for partial differential equations. Documenta Math. Extra Volume ICM 98, III 77-86 (1998)

200. Uhlmann, G.: Inverse boundary value problems and applications. Astérisque 207, 153-211 (1992)

201. Uhlmann, G.: Developments in inverse problems since Calderón's foundational paper. In: Christ, M., Kenig, C., Sadosky, C. (eds.) Harmonic Analysis and Partial Differential Equations, Chapter 19, pp. 295-345. University of Chicago Press, Chicago (1999)

202. Uhlmann, G.: Scattering by a metric. In: Pike, R., Sabatier, P. (eds.) Encyclopedia on Scattering, Chap. 6.1.5, pp. 1668-1677. Academic Press, New York (2002)

203. Uhlmann, G., Vasy, A.: Low-energy inverse problems in three-body scattering. Inverse Probl. 18, 719-736 (2002)

204. Uhlmann, G., Wang, J.-N.: Complex spherical waves for the elasticity system and probing of inclusions. SIAM J. Math. Anal. 38, 1967-1980 (2007) 
205. Uhlmann, G., Wang, J.-N.: Reconstruction of discontinuities in systems. SIAM J. Appl. Math. 28, 1026-1044 (2008)

206. Wang, J.-N.: Stability for the reconstruction of a Riemannian metric by boundary measurements. Inverse Probl. 15, 1177-1192 (1999)

207. Wiechert, E., Zoeppritz, K.: Uber erdbebenwellen. Nachr. Koenigl. Geselschaft Wiss Goettingen 4, 415-549 (1907)

208. Zhdanov, M.S., Keller, G.V.: The geoelectrical methods in geophysical exploration. In: Methods in Geochemistry and Geophysics, vol. 31. Elsevier, Amsterdam (1994)

209. Zhou, T.: Reconstructing electromagnetic obstacles by the enclosure method. Inverse Probl. Imaging 4, 547-569 (2010)

210. Zou, Y., Guo, Z.: A review of electrical impedance techniques for breast cancer detection. Med. Eng. Phys. 25, 79-90 (2003) 